\title{
Lattice string field theory: the linear dilaton in one dimension
}

\author{
Francis Bursa ${ }^{a}$ and Michael Kroyter ${ }^{b}$ \\ ${ }^{a}$ School of Physics and Astronomy, University of Glasgow, \\ Kelvin Building, University Avenue, Glasgow, G12 8QQ, United Kingdom \\ ${ }^{b}$ School of Physics and Astronomy, \\ The Raymond and Beverly Sackler Faculty of Exact Sciences, Tel Aviv University, \\ Ramat Aviv, 69978, Israel \\ E-mail: francis.bursa@glasgow.ac.uk, mikroyt@tau.ac.il
}

ABstRACT: We propose the use of lattice field theory for the study of string field theory at the non-perturbative quantum level. We identify many potential obstacles and examine possible resolutions thereof. We then experiment with our approach in the particularly simple case of a one-dimensional linear dilaton and analyse the results.

KEywords: String Field Theory, Lattice Gauge Field Theories

ArXiv EPRINT: 1405.5089 


\section{Contents}

1 Introduction $\quad 1$

1.1 String field theory 4

1.2 CFT and the linear dilaton at $d \leq 2 \quad 8$

2 Level truncation $\quad 11$

2.1 Truncation to zero $l_{0}$-level 12

$\begin{array}{lll}\text { 2.1.1 Moving the non-locality to the quadratic term } & 17\end{array}$

$\begin{array}{ll}2.2 \text { Higher levels } & 17\end{array}$

2.3 The reality condition 20

2.4 Truncation of the action to $l_{0}=1$ in scheme $4 \quad 21$

2.5 Automatization using conservation laws 24

2.6 The problem with scheme $3 \quad 25$

$\begin{array}{lll}2.7 & \text { The action in scheme } 1 & 27\end{array}$

2.8 Analytical study of the lowest mode 28

$\begin{array}{ll}2.9 & \text { Adding trivial terms to the action }\end{array}$

3 Lattice setup $\quad 30$

3.1 Observables 32

3.2 Independence of the analytical continuation on the rotation angle 33

$3.3 \quad \alpha^{\prime}$-independence 33

3.4 Estimate of statistical errors 34

4 Results $\quad 34$

$\begin{array}{lll}4.1 & \text { A single mode } & 35\end{array}$

$\begin{array}{lll}4.2 & \text { Changing } l_{\max } & 35\end{array}$

$\begin{array}{lll}\text { 4.2.1 Extensive study at } L=20 & 36\end{array}$

$\begin{array}{lll}4.2 .2 & \text { Results at } L=10 & 37\end{array}$

4.2.3 Including a large number of modes at $L=30 \quad 38$

$\begin{array}{ll}4.3 \text { Changing } x_{\text {min }} & 40\end{array}$

$\begin{array}{lll}\text { 4.3.1 } & \text { Extensive study at } L=20 & 40\end{array}$

4.3.2 Results at $L=10 \quad 42$

4.4 Small interval length 43

4.4.1 $L=6 \quad 44$

$\begin{array}{lll}4.4 .2 L=4 & 46\end{array}$

$\begin{array}{lll}4.4 .3 L & =3.15 & 46\end{array}$

4.5 Comparing different interval lengths 47

4.6 Relations among different expectation modes $\left\langle T_{n}\right\rangle \quad 50$

$\begin{array}{lll}4.6 .1 & T(x) \text { in position space } & 52\end{array}$

4.6.2 Correlations 53

$\begin{array}{lll}4.7 & \text { Varying the dilaton gradient } & 54\end{array}$ 
4.7.2 $L=10 \quad 55$

$\begin{array}{lll}\text { 4.7.3 Interpretation } & 56\end{array}$

4.8 Adding trivial parts to the action 58

4.9 Scheme $1 \quad 60$

$\begin{array}{ll}4.10 \text { Scheme } 2 & 62\end{array}$

5 Discussion $\quad 62$

\section{Introduction}

String theory is currently the most promising candidate for the unification of all forces. Unfortunately, it is neither clear what string theory is nor even how to define it. The most common "definition" of string theory found in the literature uses scattering amplitudes that are obtained from world-sheet perturbation theory. However, this perturbative expansion cannot be considered as defining a theory, since the series obtained is most probably an asymptotic one, i.e. it has a vanishing radius of convergence. This state of affairs is very similar to the one in field theory, where the Feynman diagrams themselves cannot be considered as a definition of a theory, but the field theory action, from which they are derived, does define a theory. One could hope that something similar could be achieved for string theory, which would be defined as a field theory of first quantized strings. ${ }^{1}$ The world-sheet expressions for the scattering amplitudes would then be derived from the field theory action using standard perturbation theory methods. This approach towards the definition of string theory goes under the name of string field theory.

Furthermore, string field theory should, at least in principle, be good not only for defining string theory, but also for studying string theory when the world-sheet tools are less adequate. This is completely analogous to the case of standard field theory, when one cannot rely on the standard perturbative approach at strong coupling, high temperature or high density. Of course, some of the most interesting questions one can pose relate to such regimes. In string theory one could hope that string field theory would be useful for the study of many questions, among which we can find:

- The identification of phases of string theory at large coupling and temperature, the phase transitions of the theory and their type.

- The examination of consistency and stability of string theory compactified to different dimensions and of more general, e.g. non-geometric string theory backgrounds.

- The study of solitons, in particular D-branes, time-dependent solutions, and other classical objects.

\footnotetext{
${ }^{1}$ See e.g. [1] for an introduction to string theory and the reviews [2-4] for an introduction to string field theory.
} 
- The study of various quantum effects, such as the scale-dependence of masses and couplings, which are not protected by supersymmetry.

- A particularly ambitious task would be the study of (portions of) the string theory landscape. A particular example could be the understanding of the landscape that is related to changes of the open string background.

- The study of known string theory dualities and the identification of new ones.

While other approaches towards the non-perturbative definition of string theory also exist, string field theory is very natural in principle and construction of such theories was attempted already in the first days of string theory $[5,6]$. We now have several such formulations. Among these formulations, the more promising ones are those in which the theory is covariant and universal. ${ }^{2}$ Such formulations were introduced for the bosonic open string [7], for the bosonic closed string [8] and to some extent also for the open superstring [9-15] and the heterotic string [16-19]. Interesting new ideas regarding closed superstring field theory were presented in [20-22].

Initially, string field theories were put to the test by demonstrating that they lead to the same scattering amplitudes as the world-sheet theory, i.e. one attempted to demonstrate that a proper single covering of the relevant moduli space is achieved and that the perturbative expansion for the amplitudes is correctly reproduced [23, 24]. For quite some time such perturbative studies formed the main focus for research in the field. This state of affairs changed following the realization that Sen's conjectures $[25,26]$ can be studied using string field theory, i.e. that a field theoretical approach is a most adequate one for studying non-perturbative classical solutions, in particular solutions describing the condensation of the open string tachyon. Following the first attempts to address these questions in the cases of the bosonic string [27, 28] and the superstring [29], the interest of the community drifted towards the study of such classical solutions. This led to a large body of work, which culminated with the construction of the first non-trivial analytical solution to string field theoretical equations of motion by Schnabl [30] (see also [31-33]). The new tools that were developed for the construction of this tachyon vacuum solution were further used for the construction of other analytical solutions, including the construction of simpler tachyon vacuum solutions [34], similar superstring field theory solutions [35-38] and to solutions describing marginal deformations [39-47], as well as to much further advance in the field.

Despite all this progress, a non-perturbative quantum mechanical study of string field theory was never performed. Such a study could be useful for addressing the important question of distinguishing "bare" string field theories from "effective" ones [48]. The latter ones being theories that, while capable of reproducing the correct scattering amplitudes, do not make sense as quantum theories at the non-perturbative level, since there is no way to regularize or re-sum their perturbation series. A related but simpler problem that might

\footnotetext{
2 "Universality" here refers to the property of having the same functional definition regardless of the background. This is "almost" as good as background independence. Universal formulations usually depend on the BRST world-sheet quantization of the string, e.g. in the bosonic case they depend on the $b c$-ghosts in addition to the usual space-time degrees of freedom.
} 
also benefit from such a study is that of the gauge invariance and gauge fixing of string field theories: while it was demonstrated that in a specific gauge Witten's open bosonic string field theory reproduces the correct covering of moduli space, the quantum master equation of this theory, which would ensure gauge invariance at the quantum level, is singular [49]. The situation with many other open string field theories seems to be similar. Many other issues that cannot currently be examined, such as the fate of the closed string tachyon in open string field theories could also be examined.

Another important motivation for such a study is that it could enable us to address the big challenges that we listed in the beginning of this introduction. Thus, such a study could be of use to the general string theory and high energy community, as it would significantly extend the usefulness of string field theory to the general research in the field. This could be particularly important, as string field theoretical research tends much to concentrate around string field theory itself.

However, the quantum non-perturbative study of string field theory is an enormous endeavour. In this work we attempt a first small step towards this goal. We consider the simplest possible string field theory, namely Witten's bosonic open string field theory and examine the possibility of studying this theory using lattice field theoretical methods. Our aim is to provide a proof of concept for a lattice approach to string field theory by identifying the many obstacles such an approach would have, suggesting various ways to deal with these difficulties, and examining these issues "experimentally" by lattice simulations of a particularly simple setup.

The motivation for a lattice study is the complexity of the theories that we are interested in. Even for regular field theories there is not much that can be said analytically about the quantum non-perturbative regime without, e.g. supersymmetry. Furthermore, even in the latter cases there are many aspects of the theory that cannot be addressed analytically. Given the limitations of analytical studies, it is only natural to consider a numerical approach that is adequate for the study of non-perturbative physics of field theories. Among the various possibilities, the lattice approach [50-57] is probably the most established and the most useful one.

The choice of Witten's theory among the various possibilities is also easily motivated, as it is the simplest and the most well understood among the universal string field theories. Unlike the closed string field theories it relies on a single product that can be explicitly expressed in terms of known coefficients describing the coupling of various fields. Moreover, studying the bosonic theory enables us to avoid the various complications related to properly choosing picture numbers of string fields, from which superstring field theories suffer.

As was already mentioned, there are several difficulties with the proposed approach. Let us mention here a couple of obvious ones, and postpone the discussion on the other ones to latter sections. Witten's theory is cubic, implying that the action is unbounded from below. While this is usually attributed to the unphysical nature of many of the component fields, it is bound to lead to problems for a lattice simulation. We attempt to resolve this problem using analytical continuation from a setup, to be defined in what follows, in which the cubical terms are purely imaginary. We thus trade the instability by a convergent oscillatory behaviour. 
Another complication of Witten's theory comes from the fact that the bosonic theory in the critical dimension, as well as in any other dimension above two is presumably not well defined, due to the presence of the closed string tachyonic instability. Moreover, running lattice simulations in the critical $d=26$ dimensions seems hopeless from a computational perspective. A possible way to overcome both these problems is to study linear dilaton backgrounds with $d \leq 2$. In this paper we focus mainly on the simplest one among all these models, namely the $d=1$ case. While the $d \leq 2$ theories are not really "stringy" ones, it should be possible to generalize the (universal) string field theoretical language used here to the more involved cases.

The rest of this paper is organised as follows: in the remainder of this section we introduce the reader to the basics of Witten's string field theory and to the formulation of string theory in a linear dilaton background. In particular we define the "level" of a string field and briefly introduce the notion of level truncation. We also introduce four "schemes", which we study in latter sections, for addressing the gauge symmetry of string field theory. In section 2 we further dwell upon the main tool used in the numerical study of string field theory, namely level truncation, and explain how to use it in the current context for the various schemes. We also remind of the reality condition obeyed by the string field and perform an analytical evaluation for the case of a single mode. Then, in section 3 we explain how to put the string field theory expressions on a lattice using the analytical continuation mentioned above. We introduce observables whose dependence on various parameters can be studied, and discuss some difficulties related to simulations involving a complex action. The results of the lattice simulations are given in section 4. Dependence of the observables on several parameters is studied and further understanding of numerical and computational challenges in this approach is achieved. Some concluding remarks are offered in section 5 .

\subsection{String field theory}

String field theory is a second quantization approach to string theory. The classical string field is identified with the quantum Fock space of the first quantized (world-sheet) string theory. The world-sheet theory is a two dimensional conformal field theory (CFT). Thus, its Fock space is infinite dimensional. Hence, the string field is an infinite sum of regular (component) fields. The string field is assumed to be real. The reality condition for the string field translates to reality conditions on the component fields, to be described in 2.3.

The action of the string field is,

$$
S \equiv S_{2}+S_{3}=-\int\left(\frac{1}{2 \alpha^{\prime}} \Psi \star Q \Psi+\frac{g_{o}}{3} \Psi \star \Psi \star \Psi\right),
$$

where $\Psi$ is the string field and the star product, the integral, and $Q$ are defined below. The constant $\alpha^{\prime}$, related to the string tension, defines the string length, a natural length scale for the string,

$$
l_{s} \equiv \sqrt{\alpha^{\prime}},
$$

and $g_{o}$ is the open string coupling constant. A rescaling of $\Psi$ by $g_{o}$ would result in a global prefactor of $g_{o}^{-2}$ in front of the action. Note, however, that the way we define it here, $g_{o}$ is 
a dimensionful parameter. Hence, we cannot expect to obtain canonical normalizations for the component fields both in the way the action is written here and after dividing by $g_{o}$. Jumping ahead to the equations that will follow upon using level truncation we see that in the current form of the action, canonical dimension for the scalar tachyon field is obtained in (2.10). Then, we infer from (2.19) that $g_{o}$ has a mass dimension of $\frac{6-d}{2}$. Thus, we leave the action in the form (1.1).

In order to make sense out of the action (1.1), the entities that appear in it should first be defined. The bi-linear star product takes two string fields and gives back a single string field. It has the geometric interpretation of gluing the right half of the first string with the left half of the second string. Hence, it is a non-commutative, associative product. The introduction of the star product turns the space of string fields into an algebra. The integral symbol represents "integration over the space of string fields". It is performed by gluing of the left half of a single string to its right half, followed by the evaluation of the CFT expectation value of the resulting configuration. The kinetic term is produced using the operator $Q$, which is the BRST charge of the world-sheet theory. It is given by a contour integral of a current $J$ around the state $\Psi$ in the CFT. An important property of $Q$ is that it is an odd derivation with respect to the other two operations,

$$
\begin{aligned}
\int Q \Psi & =0 \quad \forall \Psi, \\
Q\left(\Psi_{1} \star \Psi_{2}\right) & =\left(Q \Psi_{1}\right) \star \Psi_{2}+(-)^{\Psi_{1}} \Psi_{1} \star\left(Q \Psi_{2}\right) .
\end{aligned}
$$

Here $(-)^{\Psi_{1}}$ represents the parity of the string field $\Psi_{1}$. The physical string field is odd and leads to a minus sign in the definition above, while the string field that plays the role of a gauge parameter is even.

The fact that the string field includes an infinite amount of component fields is a subtlety that any numerical method should address. A common way to deal with the infinite amount of fields is to truncate the string field to a finite sum by considering only fields whose "level" is below some value $l_{1}$ and terms in the action integral, which are below some $l_{2}[58,59]$. This is referred to as a truncation to level $\left(l_{1}, l_{2}\right)$. The level of a field is defined to be its conformal weight plus a constant that sets the zero-momentum lowest level state to $l=0$. Since the Virasoro operator $L_{0}$, which reads the conformal weight of a state, serves as the (gauge fixed) kinetic term for the string field, the level $l_{0}$ is essentially the on-shell mass of the string excitation considered. Hence, level truncation has the natural physical interpretation of considering only low-mass states.

The level is invariant under the action of $Q$. However, the star product mixes different levels: the star product of string fields that were truncated to a given level results in expressions that are not truncated to this level. Hence, after the evaluation of the action in terms of component fields, the action should also be truncated. As $l_{1,2}$ are sent to infinity one expects to obtain the result of using the full string field. There is no proof that this should work and subtleties might well arise. However, in the past it always did work. The inclusion of a kinetic term implies $l_{2} \geq 2 l_{1}$ while the cubicity of the action implies $l_{2} \leq 3 l_{1}$. In practice one always works either in the $(l, 2 l)$ (which is simpler - has far fewer terms) or the (more "physical") $(l, 3 l)$ level-truncation. 
The conformal dimension of a field depends on its momentum. Most past papers considered only the zero momentum sector. Those who did consider non-zero momentum either considered the double limit of a truncation in which the zero-momentum level and the momentum were considered separately, or took the more physical choice of considering the total conformal weight as a single level parameter. In both cases, the only allowed momentum was along a compactified space-like direction. Hence, this momentum was quantized and its contribution to the level was always positive, which is sensible from the perspective of level truncation. This is not quite the case that we consider. We do not have compactified directions and we consider the most general space-time dependence of the fields. However, the use of a lattice implies that we have to Wick-rotate the time direction and to evaluate the action on a finite range of space-time, with some arbitrary (Neumann/Dirichlet) boundary conditions. These two restrictions turn the use of the more physical level truncation into a sensible choice, which we adopt. Not only would that free us from considering double limits, but it would also simplify and make more accurate the consideration of the string-field-theory-inherent non-localities. Thus, we define the total level of a string field to be

$$
l=l_{0}+l_{p},
$$

where $l_{0}$ is defined by the mass of the specific excitation and $l_{p}$ includes the contribution of the momentum to the conformal weight.

Another important issue is that of the space of string fields, a proper definition of which is still lacking. Currently it is not even clear which mathematical concepts are needed in order to properly define it. However, the general problems with the definition of this space should not emerge in the context of level truncation. On the other hand, we should decide whether the space of string fields should be restricted to string fields of a given (first-quantization) ghost number and whether dependence on the ghost zero mode should be allowed in its definition.

The ghost system that we refer to here is the $b c$ system used to fix the conformal gauge symmetry on the world-sheet. This symmetry is generated by the energy momentum tensor $T(z)$ which is an even object of conformal dimension $h=2$. Thus, it is fixed by a system of two conjugate odd bosons, $b$ whose conformal dimension is also $h=2$ and $c$ with $h=-1$. The ghost number is defined as the number of $c$ insertions minus the number of $b$ insertions. These first-quantized ghosts manifest themselves in the second quantized formulation by declaring that the string field $\Psi$ is a functional not only of the space-time $X^{\mu}$ variables, but also of the $b c$ system. In terms of modes these conformal fields can be expanded $\mathrm{as}^{3}$

$$
b(z)=\sum_{n=-\infty}^{\infty} \frac{b_{n}}{z^{n+2}}, \quad c(z)=\sum_{n=-\infty}^{\infty} \frac{c_{n}}{z^{n-1}} .
$$

The fact that these fields are canonically conjugate implies ${ }^{4}$

$$
\left[b_{n}, c_{m}\right]=\delta_{n,-m}
$$

\footnotetext{
${ }^{3}$ Our conventions and world-sheet analysis follow Polchinski's textbook [1].

${ }^{4}$ In this paper the brackets represent the graded commutator, which for the current case is an anticommutator.
} 
The world-sheet quantum space is defined in terms of vertex operators, which are restricted to carry ghost number one. The classical string field $\Psi$ generalizes the space of vertex operators and should therefore also be restricted to carry ghost number one. However, a proper treatment of the gauge invariance of the classical action (1.1), can modify this restriction. The gauge transformation that leaves the action invariant is

$$
\delta \Psi=Q \Lambda+\alpha^{\prime} g_{o}(\Psi \star \Lambda-\Lambda \star \Psi) .
$$

A common way to classically fix the gauge is to impose the Siegel gauge, which is a string field theoretical extension of the Feynman gauge for the vector component field. This gauge choice is enforced by requiring that the $b$-ghost zero mode annihilates the string field,

$$
b_{0} \Psi=0
$$

The Fock vacuum is annihilated by $b_{0}$. Hence, the Siegel gauge can also be defined as the space of states built from the vacuum without using $c_{0}$. A quantum treatment of the gauge symmetry should take into account the fact that the gauge symmetry is redundant and "uses the equations of motion". The most natural framework for addressing such a system covariantly is the field-antifield BV formalism. This formalism was applied to Witten's string field theory in [60-62]. The result is very elegant: prior to gauge fixing the action should be replaced by a "master action", which is identical in form to the classical action (1.1), except that the string field $\Psi$ should no longer be constrained to carry ghost number one. ${ }^{5}$ One can gauge fix the master action to obtain the Siegel gauge in the space of string fields with unrestricted ghost number. A potential subtlety with the master action is that according to the general BV formalism it should obey the "quantum master equation". However, it is still not clear if this is the case and it is only certain that it obeys the "classical master equation".

While gauge fixing is necessary in perturbation theory, this is not always the case in a lattice approach, in which the infinite gauge orbits are replaced by finite ones. Hence, we can consider the following four options for our space of string fields, to which we refer in the following as schemes:

1. Classical string fields without gauge fixing, i.e. $\Psi$ carries ghost number one but has no ghost zero-mode restrictions.

2. Classical gauge-fixed string fields. This is probably inconsistent, since there is no justification for gauge fixing without a proper treatment of the gauge symmetry.

3. BV string field without gauge fixing. Here, all ghost numbers are considered and the string field is allowed a $c_{0}$ dependence.

4. Gauge-fixed BV string field, i.e. $\Psi$ carries all possible ghost numbers but is $c_{0}$-independent.

\footnotetext{
${ }^{5}$ The string field should still be an odd object. Hence, the component fields at even ghost numbers have to be odd. This is also consistent with the general parity assignments of the BV/BRST formalisms.
} 
One of the important advantages of the lattice approach to field theory is that it provides a regulator that does not break gauge symmetry. In our case, on the other hand, the lattice does break the gauge symmetry, since the star product mixes all levels. Hence, one should expect that the gauge fixed schemes, in which the gauge symmetry was already taken care of, would be better behaved. While we will experiment with all the four schemes, we will see that, as we expect, scheme 4 seems to be the most promising one.

\subsection{CFT and the linear dilaton at $d \leq 2$}

The first step towards the explicit construction of the string field theory is the choice of the background CFT. In string theory the background always includes the $b c$ ghost system, which we already described. Other than the ghost system the world-sheet theory depends also on a matter system, which can be any CFT, as long as its central charge is $c_{m}=26$. This value for the central charge is needed in order to cancel the central charge of the ghost system which is $c_{\mathrm{gh}}=-26$. The vanishing of the total central charge is necessary for avoiding conformal anomalies.

In this paper, we work with a one dimensional linear dilaton theory. But let us for now consider the more general case of a linear dilaton in $d \leq 2$ dimensions. ${ }^{6}$ This case includes theories with $1<d<2$, where we identify the dimension $d$ with the central charge of the non-linear-dilaton part of the matter sector plus one. This identification stems from the fact, on which we elaborate below, that the linear dilaton can be realized by a single non-homogenous direction, while standard space-time directions correspond to one unit of central charge. The $1<d<2$ theories can be realized using non-minimal models, but we shall refrain in this work from going into details about these systems. For the two dimensional case note that while we Wick-rotate for enabling the lattice simulations, there is still a distinction between the two dimensions, due to the linear dilaton background. Also note that the dilaton gradient, $V^{\mu}$ to be defined below, is of the order of the string scale,

$$
m_{s} \equiv l_{s}^{-1}=\left(\alpha^{\prime}\right)^{-\frac{1}{2}}
$$

The string scale is usually identified (presumably up to a constant of order one or around it) with the Planck mass. Hence, these spaces differ substantially from standard space-times. We will further have to set two other scales, namely the lattice spacing and the lattice size. In order to obtain a proper description of the physics, the first one should be sent to zero and the second one should be sent to infinity, in $l_{s}$ units, in order to approach the continuum limit.

\footnotetext{
${ }^{6}$ In the two dimensional case, the world-sheet degrees of freedom are the "tachyon" field and the "discrete states", which are physical only for specific momenta. Scattering amplitudes of both tachyons and discrete states are known $[63,64]$. They were considered from the string field theory perspective in [65-67]. Other relevant papers include the identification of the ground ring/ $W_{\infty}$ structure [68], the introduction of ZZ [69] and FZZT D-branes [70, 71], open/closed duality and relation to the supersymmetric theories [72] and the decay of ZZ-branes [73].
} 
In a theory of open strings, the matter fields can be expanded as, ${ }^{7}$

$$
X^{\mu}(z, \bar{z})=x^{\mu}-i \alpha^{\prime} p^{\mu} \log |z|^{2}+i \sqrt{\frac{\alpha^{\prime}}{2}} \sum_{n=1}^{\infty}\left(\frac{a_{n}^{\mu}}{\sqrt{n}}\left(z^{-n}+\bar{z}^{-n}\right)-\frac{a_{n}^{\dagger \mu}}{\sqrt{n}}\left(z^{n}+\bar{z}^{n}\right)\right),
$$

where $\mu=1,2$ in the $2 d$ case and takes a single value and hence can be omitted in the $1 d$ case. Taking the derivative with respect to $z$ gives

$$
i \partial X^{\mu}(z)=\frac{\alpha^{\prime} p^{\mu}}{z}+\sqrt{\frac{\alpha^{\prime}}{2}} \sum_{n=1}^{\infty} \sqrt{n}\left(a_{n}^{\mu} z^{-n-1}+a_{n}^{\dagger \mu} z^{n-1}\right) .
$$

These fields have the following operator product expansion (OPE)

$$
X^{\mu}(z, \bar{z}) X^{\nu}(w, \bar{w}) \sim-\frac{\alpha^{\prime}}{2} \eta^{\mu \nu} \log |z-w|^{2},
$$

which translate into the standard commutation relations among the infinite set of creation and annihilation operators,

$$
\left[a_{n}^{\mu}, a_{m}^{\dagger \nu}\right]=\eta^{\mu \nu} \delta_{m, n}
$$

We assume a flat (space-time) metric,

$$
\begin{array}{rlrl}
\eta^{\mu \nu} & =\operatorname{diag}(1,1) & d & =2, \\
\eta & =1 & d & =1 .
\end{array}
$$

Momentum states are represented as usual using the operators $e^{i k \cdot X}$. The OPE (1.13) implies that these exponents suffer from short distance singularities and should be normalordered. We do not write the normal ordering symbol and assume that all fields that we consider are implicitly normal ordered. In the standard flat background with constant dilaton, the normal-ordered exponents carry well-defined conformal weights,

$$
(h, \bar{h})=\left(\frac{\alpha^{\prime} k^{2}}{4}, \frac{\alpha^{\prime} k^{2}}{4}\right) .
$$

Since we consider open strings, our fields are given by insertions on the boundary of the CFT theory, which we identify with the real axis,

$$
\Im(z)=0 \quad \Longleftrightarrow \quad z=\bar{z},
$$

and the CFT is defined on the upper half plane. One usually extends the CFT to the whole complex plane using the doubling trick, according to which $z$-dependence above the real line is identified with $\bar{z}$-dependence below it. Still, the real line is special, e.g. conformal fields may suffer from extra singularities when approaching it, originating from collisions of $z$ and $\bar{z}$. In particular, momentum states should now be normal-ordered according to the boundary-normal-ordering. Then, they carry a well defined single conformal weight

$$
h\left(e^{i k \cdot X}\right)=\alpha^{\prime} k^{2} .
$$

\footnotetext{
${ }^{7}$ In the literature it is common to find the variables $\alpha_{n}^{\mu} \equiv \sqrt{|n|} a_{n}^{\mu}$.
} 
Another confusion that can arise from the presence of the boundary is the relation between the derivative with respect to $z$ and that with respect to the boundary parameter. We will try to avoid this issue by always considering only the $z$ variable.

In order to fully describe the CFT one has to define the energy-momentum tensor,

$$
T(z)=T^{g}(z)+T^{m}(z),
$$

where the superscripts stand for "ghost" and "matter". The ghost part of the energy momentum tensor is fixed,

$$
T^{g}(z)=-\partial b c(z)-2 b \partial c(z),
$$

but the matter part is theory-dependent. In our case it equals,

$$
T^{m}(z)=-\frac{1}{\alpha^{\prime}} \partial X^{\mu} \partial X_{\mu}+V^{\mu} \partial^{2} X_{\mu} .
$$

The second "improvement" term encodes the linear dilaton nature of the background. This term changes the central charge to a new value,

$$
c=d+6 \alpha^{\prime} V^{2} .
$$

In order to obtain the correct value of 26 for the central charge, one has to impose

$$
V^{2}=\frac{26-d}{6 \alpha^{\prime}} .
$$

In particular, the cases we are interested in are,

$$
\begin{aligned}
& V^{\mu}=(0, V), \quad V=-\frac{2}{\sqrt{\alpha^{\prime}}} \quad d=2, \\
& V=-\sqrt{\frac{25}{6 \alpha^{\prime}}} \quad d=1
\end{aligned}
$$

where the minus sign is just a convention.

In a linear-dilaton background the following modifications occur as compared to a standard flat-space background $[74,75]$ :

- The momentum-conservation delta function is modified in order to reflect the breakdown of translational invariance,

$$
\delta^{d}\left(\sum_{n} k_{n}^{\mu}\right) \longrightarrow \delta^{d}\left(\sum_{n} k_{n}^{\mu}+i V^{\mu}\right) .
$$

Here, the delta function is formally defined by,

$$
\delta^{d}\left(\sum_{n} k_{n}^{\mu}+i V^{\mu}\right) \equiv \frac{1}{(2 \pi)^{d}} \int d^{d} x e^{-i x \cdot \sum k_{n}+x \cdot V} .
$$


- The field $X$, aligned with the linear dilaton direction, is no longer a (logarithmic) conformal tensor,

$$
X^{\mu}(z, \bar{z}) \rightarrow f \circ X^{\mu}=X^{\mu}(f(z), \bar{f}(\bar{z}))+\frac{\alpha^{\prime}}{2} V^{\mu} \log \left|f^{\prime}(z)\right|^{2} .
$$

The momentum operators built from $X^{\mu}$ remain conformal tensors. However, their conformal weights change,

$$
h\left(e^{i k \cdot X}\right)=\alpha^{\prime}\left(k^{2}+i k \cdot V\right) .
$$

- The change in $T^{m}$ induces a change in $Q$, since the following identity holds in general

$$
J(z)=c T^{m}(z)+b c \partial c(z)+\frac{3}{2} \partial^{2} c(z)
$$

where $J(z)$ is the BRST current, which should be integrated to obtain $Q$.

The introduction of a linear dilaton introduces complications also from the lattice perspective. Specifically, the fact that the target space is no longer homogenous implies that it would not be enough to consider the length $L$ of the lattice, as a single length parameter. Instead, we would have to study the dependence on $x_{\min }$ and $x_{\max }$, the minimum and maximum points of the spatial direction ${ }^{8} X$, or alternatively on $x_{\min }$ and the lattice interval

$$
L \equiv x_{\max }-x_{\min } .
$$

\section{Level truncation}

In this section we implement level truncation in order to describe various aspects, problems and resolutions of our program from the string field theory perspective. We evaluate most expressions in an arbitrary dimension and concentrate on the $d=1$ case at the end. Later on, in section 3, we discuss the computational lattice perspective. Here we start by evaluating the action for $l_{0}=0$ in 2.1. Next we discuss the way by which higher levels can be added in 2.2 and explain how to impose the reality condition in 2.3. Then, we describe explicitly the first case of a higher level, namely $l_{0}=1$, in 2.4. In order to go to higher levels one has to define a systematic way for the evaluation of the very many terms that are present in the action. We sketch a method that can be used for an automatization of the evaluation of the action in 2.5 and utilize it in 2.6 for the evaluation of the action of scheme 3 at $l_{0}=1$. We further explain there that there is a problem with this action that prevents us from using scheme 3 together with level truncation, after which we truncate the scheme 3 action to a scheme 1 action in 2.7. We end this section by an analytical study of the simplest possible case, that of a single mode, in 2.8 , followed by a discussion of one more potential obstacle towards using our methods, in 2.9 .

\footnotetext{
${ }^{8}$ Here, it is assumed that we work in $d=1$. For $d>1, x_{\min }$ and $x_{\max }$ are the minimum and maximum values in the direction of the linear dilaton.
} 


\subsection{Truncation to zero $l_{0}$-level}

We consider classical string fields that are built upon the vacuum of the first quantized theory,

$$
|\Omega\rangle \equiv c(0)|0\rangle=c_{1}|0\rangle,
$$

where $|0\rangle$ is the $\operatorname{SL}(2)$ invariant vacuum. ${ }^{9}$ The vacuum $|\Omega\rangle$ is odd and carries ghost number one, as is proper for a classical string field. General string field configurations are built from this vacuum by acting on it with the operators $b_{-n}$ with $n>0, c_{-n}$ with $n \geq 0$ and $a_{n}^{\mu}$ with $n>0$. Note that the $b_{-n}$ and $c_{-n}$ ghost operators are odd, so each one of them can act at most once, while the $a_{n}^{\mu}$ are even and so each one of them can act indefinitely. In order to retain the restriction to ghost number one, which is needed for schemes 1 and 2 , the number of $b$ and $c$ insertions must be the same. For the other two schemes there is no such restriction. However, if the total number of $b$ and $c$ ghost insertions is odd, the relevant component field must also have odd parity in order not to change the parity of the string field.

The momentum of the state is quantized when we work with $0<X<L$. Assuming Dirichlet boundary conditions, the momentum dependence would come from $\sin \left(\frac{\pi k \cdot X}{L}\right)$, with $k$ an integer. We can now define the level as the sum of the indices of all the operators plus a contribution from the momentum, e.g. the level of $c_{-3} b_{-2}\left(a_{4}^{\dagger 1}\right)^{2}|\Omega, p\rangle$ is $l=l_{0}+l_{p}$, with $l_{0}=3+2+4+4=13$. However, (1.30) implies that the sine factor is not an eigenvalue of the level operator. Not only that, but the eigenvalues of the two distinct exponents composing the sine are complex. We resolve this problem below.

At the lowest (zero) $l_{0}$-level the string field contains only two component fields. We impose the reality condition on the string field. This implies that the following two component fields are real,

$$
\Psi_{0}=\int d^{d} p\left(T(p) e^{i p \cdot X}+\mathcal{T}(p) e^{i p \cdot X} c_{0}\right)|\Omega\rangle .
$$

The first of these fields is the "tachyon" $T$ (not to be confused with the energy-momentum tensor). It carries no $c_{0}$ insertion and has ghost number one and so contributes in all four schemes. The second field $\mathcal{T}$ is odd. It carries ghost number two and contains the $c$ zero mode in its definition. Hence, it contributes only in scheme number three. In any case, it cannot contribute to the action without the presence of string fields of ghost number less than one. Thus, it does not contribute at all at zero $l_{0}$-level and is set for now to zero. Direct calculation shows that,

$$
Q \Psi_{0}=\int d^{d} p T(p) \alpha^{\prime}\left(\left(p+\frac{i V}{2}\right)^{2}+m_{0}^{2}\right) \partial c c e^{i p \cdot X}|\Omega\rangle,
$$

where we defined,

$$
m_{0}^{2} \equiv \frac{V^{2}}{4}-\frac{1}{\alpha^{\prime}}
$$

\footnotetext{
${ }^{9}$ The conformal symmetry is generated by the Virasoro operators $L_{n}, n \in \mathbb{Z}$. The vacuum $|0\rangle$ is invariant under an SL(2) subalgebra of the Virasoro algebra generated by $L_{0}$ and $L_{ \pm 1}$. This subalgebra becomes useful for us already at $l_{0}=1(2.40)$. The other Virasoro operators are useful at higher levels.
} 
We see that the constant term inside the parentheses vanishes only for the value of $V(1.24)$ at $d=2$. This constant fixes the mass of this mode. Hence, we see that the tachyon becomes massless exactly in two dimensions, while for $d=1$ it is massive.

The kinetic term of the tachyon reads,

$$
S_{2}=-\frac{1}{2 \alpha^{\prime}} \int \Psi_{0} Q \Psi_{0}=-\frac{1}{2 \alpha^{\prime}}\left\langle\left(I \circ \Psi_{0}\right)(0) Q \Psi_{0}(0)\right\rangle,
$$

where the expectation value is evaluated in the upper half plane and $I$ is the conformal transformation,

$$
I(z)=-\frac{1}{z}
$$

For the evaluation of (2.5) we have to regularize, $z \rightarrow \epsilon$ and continue as in [76]. The expectation value factorizes into matter and ghost parts. The ghost part gives,

$$
\left\langle c\left(-\frac{1}{\epsilon}\right) c^{\prime} c(0)\right\rangle=\frac{1}{\epsilon^{2}},
$$

while for the matter part we have to evaluate

$$
\left\langle e^{i p \cdot X}\left(-\frac{1}{\epsilon}\right) e^{i q \cdot X}(0)\right\rangle=\frac{(2 \pi)^{d} \delta^{d}(p+q+i V)}{\left(\frac{1}{\epsilon}\right)^{-2 \alpha^{\prime} p \cdot q}} .
$$

Using this result for (2.2) and (2.3) leaves us with integration over the momenta, as well as with a factor coming from the conformal transformation,

$$
\left(\frac{d I}{d z}\right)^{h}=\left(\frac{1}{\epsilon^{2}}\right)^{\alpha^{\prime}\left(p^{2}+i p \cdot V\right)-1} .
$$

Using the delta function one sees that all $\epsilon$-dependent factors cancel out, regardless of the specific background and the final result is,

$$
\begin{aligned}
S_{2} & =-\frac{1}{2} \int d^{d} p d^{d} q(2 \pi)^{d} \delta^{d}(p+q+i V) T(p) T(q)\left(\left(p+\frac{i V}{2}\right)^{2}+m_{0}^{2}\right) \\
& =-\frac{1}{2} \int d^{d} p d^{d} q d^{d} x e^{-i x \cdot(p+q+i V)} T(p) T(q)\left(\left(\frac{p-q}{2}\right)^{2}+m_{0}^{2}\right) \\
& =-\frac{1}{2} \int d^{d} x e^{V \cdot x}\left(\frac{\nabla T(x) \cdot \nabla T(x)-T(x) \nabla^{2} T(x)}{2}+m_{0}^{2} T(x)^{2}\right),
\end{aligned}
$$

where we abuse the notation by using the same symbol for the Fourier conjugate fields $T(p)$ and $T(x)$.

We now have to evaluate the cubic term,

$$
S_{3}=-\frac{g_{o}}{3} \int \Psi_{0} \star \Psi_{0} \star \Psi_{0}=-\frac{g_{o}}{3} \int d^{d} p d^{d} q d^{d} k\left\langle\left(f_{-1} \circ \Psi_{0}\right)\left(f_{0} \circ \Psi_{0}\right)\left(f_{1} \circ \Psi_{0}\right)\right\rangle .
$$

The three conformal transformations are obtained by sending the upper half plane to the unit disk using,

$$
w=\frac{1+i z}{1-i z}
$$


then rescaling $w$ and relocating it to the three points of the "rotated Mercedes-Benz logo",

$$
w \rightarrow e^{\frac{2 \pi i n}{3}} w^{\frac{2}{3}}
$$

and finally sending it back to the upper half plane using the inverse of (2.12),

$$
z=i \frac{1-w}{1+w} .
$$

The only relevant information about these conformal transformations is,

$$
f_{0}(0)=0, \quad f_{ \pm 1}(0)= \pm \sqrt{3}, \quad f_{0}^{\prime}(0)=\frac{2}{3}, \quad f_{ \pm 1}^{\prime}(0)=\frac{8}{3} .
$$

Now, the ghost part contributes,

$$
\langle c(-\sqrt{3}) c(0) c(\sqrt{3})\rangle=2 \cdot 3^{\frac{3}{2}},
$$

the matter part is,

$$
\left\langle e^{i p \cdot X}(-\sqrt{3}) e^{i k \cdot X}(0) e^{i q \cdot X}(\sqrt{3})\right\rangle=\frac{(2 \pi)^{d} \delta^{d}(p+k+q+i V)}{3^{-\alpha^{\prime} p \cdot k} \cdot 3^{-\alpha^{\prime} k \cdot q} \cdot 12^{-\alpha^{\prime} p \cdot q}},
$$

and the conformal weights contribute,

$$
\begin{aligned}
& \left(\frac{d f_{-1}}{d z}\right)^{h_{-1}}\left(\frac{d f_{0}}{d z}\right)^{h_{0}}\left(\frac{d f_{1}}{d z}\right)^{h_{1}} \\
& =\left(\frac{8}{3}\right)^{\alpha^{\prime}\left(p^{2}+i p \cdot V\right)-1}\left(\frac{2}{3}\right)^{\alpha^{\prime}\left(k^{2}+i k \cdot V\right)-1}\left(\frac{8}{3}\right)^{\alpha^{\prime}\left(q^{2}+i q \cdot V\right)-1}
\end{aligned}
$$

All in all we get,

$$
\begin{aligned}
S_{3} & =-g_{o} \int d^{d} p d^{d} q d^{d} k \frac{(2 \pi)^{d} \delta^{d}(p+k+q+i V)}{3} T(p) T(k) T(q) K^{3-\alpha^{\prime}\left(p^{2}+k^{2}+q^{2}+V^{2}\right)} \\
& =-\frac{g_{o} K^{3-\alpha^{\prime} V^{2}}}{3} \int d^{d} x e^{V \cdot x} \tilde{T}(x)^{3} .
\end{aligned}
$$

Here (as usual) we defined,

$$
K \equiv \frac{3 \sqrt{3}}{4},
$$

and used the delta function in the first equality. In the second equality we moved to $x$-space, as in (2.10) and defined (as usual) the tilded variables,

$$
\tilde{T}(x)=K^{\alpha^{\prime} \nabla^{2}} T(x) .
$$

Similar relations are to be understood for other tilded variables in what follows. The prefactor $K^{3-\alpha^{\prime} V^{2}}$ can be absorbed into a redefinition of the coupling constant. Note also the linear dilaton factor $e^{V \cdot x}$, which is common to the quadratic and cubic terms. A rescaling of $T$ by this factor leads to a space-dependent effective coupling,

$$
g_{o}^{\mathrm{eff}} \sim e^{-\frac{V \cdot x}{2}} .
$$


This coupling goes from zero to infinity along the linear dilaton direction, which implies that the pre-factor $g_{o} K^{3-\alpha^{\prime} V^{2}}$ can be set to unity by an appropriate choice of the origin. Note, that this effective coupling constant is still dimensionful. It is possible to multiply it by a proper power of $\alpha^{\prime}$ in order to obtain a dimensionless coupling constant.

Let us now perform the advocated field redefinition,

$$
T(x)=e^{-\frac{V \cdot x}{2}} \tau(x) \Longleftrightarrow T(p)=\tau\left(p+i \frac{V}{2}\right) .
$$

The kinetic term takes now the standard form,

$$
S_{2}=-\frac{1}{2} \int d^{d} x\left(m_{0}^{2} \tau^{2}+(\nabla \tau)^{2}\right)
$$

This field redefinition is defined pointwise. Hence, the Jacobian is just a number and can be ignored. The interaction term transforms under the above field redefinitions into,

$$
S_{3}=-\frac{g_{o} K^{3\left(1-\frac{\alpha^{\prime} V^{2}}{4}\right)}}{3} \int d^{d} x e^{-\frac{V \cdot x}{2}} \tilde{\tau}(x)^{3} .
$$

It is easiest to obtain this expression in momentum space, where one has to use the delta function and deform the contour of integration. Note that the resulting interaction term is both space-dependent and non-local. In $p$-space the spatial-dependence and non-locality reverse their roles.

The spatial dependence of the coupling constant implies that we cannot use periodic boundary conditions on the lattice, since it would glue a strong coupling region with a weak coupling region, which is unphysical. Instead we can use, as mentioned above, Dirichlet or Neumann boundary conditions. We choose the former and evaluate the action in a box $x_{\min }^{\mu}<x^{\mu}<x_{\max }^{\mu}$, with $x_{\max }^{\mu}-x_{\min }^{\mu}=L^{\mu}$. Specifying now to $d=1$, we can expand,

$$
\tau(x)=\sqrt{\frac{2}{L}} \sum_{n=1}^{\infty} \tau_{n} \sin \left(\frac{\pi n\left(x-x_{\min }\right)}{L}\right) .
$$

We can now recognize one more advantage of $\tau$ over $T$. We mentioned above that the expansion of $T$ in terms of sine modes does not involve conformal eigenmodes and its expansion in exponents leads to complex eigenvalues. Contrary to that, one can see that the expansion in sine modes of $\tau$ is well defined and real. Furthermore, we shall see in section 2.3 that working with the $\tau$ variable is essential also in order to obey the string field reality condition.

Another issue that we would like to mention is that of the variational principle. While we do not derive here equations of motion, since we concentrate on the action itself, it could still be interesting to examine their derivation and their form in the case at hand. String field theory includes an infinite number of derivatives. It is known that there might be subtleties with the definition of the variational principle in such theories [77, 78]. One could wonder whether our choice of boundary conditions is consistent with a variational 
principle. The quadratic term is the standard one and so is its variation. The variation of the cubic term leads to

$$
\delta S_{3}=-\frac{g_{o} K^{3\left(1-\frac{\alpha^{\prime} V^{2}}{4}\right)}}{3} \int d^{d} x e^{-\frac{V \cdot x}{2}} \tilde{\tau}(x)^{2} \delta \tilde{\tau}(x) .
$$

It seems that in order to obtain an equation of motion we need to change the $\delta \tilde{\tau}(x)$ term into a $\delta \tau(x)$ term, i.e. to integrate by parts the $K^{\alpha^{\prime} \nabla^{2}}$ factor acting on $\delta \tau(x)$, such that it would act on the $\tilde{\tau}^{2}(x)$ factor and on the dilaton factor $e^{-\frac{V \cdot x}{2}}$. Such an integration by parts would lead to boundary terms that include the variation of various higher order derivatives of $\tau(x)$. These expressions should not a-priori vanish. Moreover, setting all these infinitely many terms to zero could lead to very strict functional restrictions on $\tau(x)$. However, we can take another approach. Given the expansion (2.26) we obtain,

$$
\tilde{\tau}(x)=\sqrt{\frac{2}{L}} \sum_{n=1}^{\infty} K^{-\alpha^{\prime}\left(\frac{\pi n}{L}\right)^{2}} \tau_{n} \sin \left(\frac{\pi n\left(x-x_{\min }\right)}{L}\right) .
$$

When expressed in this form it seems that $\tilde{\tau}(x)$ and $\delta \tilde{\tau}(x)$ vanish at the boundaries. Nonetheless, this assertion relies on some convergence properties of the expansion, which might not be well justified. This issue is related to the discussion in [77, 78] and more generally to the problem of properly defining the space of string fields. Attempting to analyse it would take us too far away. Hence, we do not dwell on these questions further.

We can now assign a level to the modes in the expansion above,

$$
l\left(\tau_{n}\right)=\alpha^{\prime}\left(\frac{\pi n}{L}\right)^{2} .
$$

We have to include all levels that are smaller than some $l<1$. The restriction $l<1$ comes from the fact that it was assumed that we include only the tachyon field. For $l \geq 1$ higher $l_{0}$-modes might also contribute. ${ }^{10}$ The physical origin of this restriction is that if we decide to probe lower than string-scale size modes, we should also include the higher modes, which are also of this size.

An $l$ that allows for $N$ modes is equivalent to working on an $N$-site lattice. However, the non-locality and space-dependence of the action simplify if we perform the analysis directly in terms of the modes. The free part of the action is now,

$$
S_{2}=-\frac{1}{2} \sum_{n=1}^{N}\left(\frac{1}{24 \alpha^{\prime}}+\left(\frac{\pi n}{L}\right)^{2}\right) \tau_{n}^{2} .
$$

Assuming that we work in the $(l, 3 l)$ scheme (that is, if all interaction terms are to be included), the interaction term reads,

$$
\begin{aligned}
S_{3}= & -\frac{g_{o} K^{3\left(1-\frac{\alpha^{\prime} V^{2}}{4}\right)}}{3} \sum_{n_{1,2,3}=1}^{N} K^{-\alpha^{\prime}\left(\frac{\pi}{L}\right)^{2}\left(n_{1}^{2}+n_{2}^{2}+n_{3}^{2}\right)} \tau_{n_{1}} \tau_{n_{2}} \tau_{n_{3}} f_{n_{1}, n_{2}, n_{3}}, \\
f_{n_{1}, n_{2}, n_{3}} \equiv & \left(\frac{2}{L}\right)^{\frac{3}{2}} \int_{x_{\min }}^{x_{\max }} d x e^{-\frac{V x}{2}} . \\
& \cdot \sin \left(\frac{\pi n_{1}\left(x-x_{\min }\right)}{L}\right) \sin \left(\frac{\pi n_{2}\left(x-x_{\min }\right)}{L}\right) \sin \left(\frac{\pi n_{3}\left(x-x_{\min }\right)}{L}\right) .
\end{aligned}
$$

\footnotetext{
${ }^{10}$ For a Dirichlet expansion these modes actually contribute starting at some $l>1$.
} 
We can substitute $-\frac{V x}{2}=\sqrt{\frac{25}{24 \alpha^{\prime}}} x$. We left the $V$ dependence for enabling the evaluation of this expression with unphysical values of $V$. We can now evaluate this action on the lattice. Note, that our Wick-rotation was performed in such a way that we have to consider,

$$
Z=\int\left(\prod_{n} d \tau_{n}\right) e^{S} .
$$

\subsubsection{Moving the non-locality to the quadratic term}

In previous studies of level-truncated string field theory it was suggested to move the nonlocality from the cubic term to the quadratic term. The motivation was the simplification of the (relatively more complicated) interaction term. Furthermore, as it is moved to the quadratic term, the non-locality becomes completely diagonal, which results in somewhat simplified expressions.

At the lowest $l_{0}$ level, the action is still given by the sum of (2.24) and (2.25), only now the fundamental field, which should be expanded in modes is $\tilde{\tau}$, while $\tau$ is defined in terms of it as

$$
\tau=K^{\alpha^{\prime} p^{2}} \tilde{\tau}
$$

For simplicity of notations, we drop the tilde from now on, with the understanding that the correct variables are used.

Expanding in the modes for the tachyon field, the action is modified to

$$
\begin{aligned}
& S_{2}=-\frac{1}{2} \sum_{n=1}^{N} K^{-2 \alpha^{\prime}\left(\frac{\pi n}{L}\right)^{2}}\left(\frac{1}{24 \alpha^{\prime}}+\left(\frac{\pi n}{L}\right)^{2}\right) \tau_{n}^{2}, \\
& S_{3}=-\frac{g_{o} K^{3\left(1-\frac{\alpha^{\prime} V^{2}}{4}\right)}}{3} \sum_{n_{1,2,3}=1}^{N} \tau_{n_{1}} \tau_{n_{2}} \tau_{n_{3}} f_{n_{1}, n_{2}, n_{3}},
\end{aligned}
$$

where the definition of $f_{n_{1}, n_{2}, n_{3}}$ did not change.

As higher level fields are added, one can similarly decide whether the better representation is the one in which the untilded fields are the fundamental ones, or the one with the tilded fields. The needed manipulations are completely analogous to what is done here.

\subsection{Higher levels}

Evaluating conformal transformations can be tedious, especially at higher levels, where the coefficient fields are not represented by primary conformal fields. A way to simplify calculations was actually devised even before the CFT formulation of [76]. In this formulation the action is given by,

$$
S=-\frac{1}{2 \alpha^{\prime}}{ }_{12}\left\langle V_{2}|| \Psi\right\rangle_{1}|Q \Psi\rangle_{2}-\frac{g_{o}}{3}{ }_{123}\left\langle V_{3}|| \Psi\right\rangle_{1}|\Psi\rangle_{2}|\Psi\rangle_{3},
$$

where the subscripts represent an index of a copy of the Hilbert space. The two-vertex $V_{2}$ and three-vertex $V_{3}$ live in the spaces $H^{2}$ and $H^{3}$ respectively. They are squeezed states and their form for flat background was found in [79-84]. The modification of these works 
to a linear dilaton background is relatively simple, due to the similarity with the bosonized ghost sector, studied in these papers. Explicit expressions for $d=2$ were given in [66]. Both factorize into matter and ghost sectors (the matter sector further factorizes into $d$ independent sectors).

The two-vertex is explicitly given by,

$$
\begin{aligned}
{ }_{12}\left\langle V_{2}^{m}\right|= & \int d^{d} p d^{d} q_{1}\langle p|{ }_{2}\langle q| \delta^{d}(p+q+i V) . \\
& \cdot \exp \left(\sum_{n=1}^{\infty}(-1)^{n+1}\left(a_{n}^{\mu}\right)^{1}\left(a_{n}^{\mu}\right)^{2}\right) \\
{ }_{12}\left\langle V_{2}^{g}\right|= & { }_{12}\langle\Omega|\left(c_{0}^{1}+c_{0}^{2}\right) \exp \left(\sum_{n=1}^{\infty}(-1)^{n}\left(b_{n}^{1} c_{n}^{2}+b_{n}^{2} c_{n}^{1}\right)\right) .
\end{aligned}
$$

The superscripts $m$ and $g$ in these expressions stand for "matter" and "ghost". The superscripts 1 and 2 over the oscillators represent the two spaces.

In order to evaluate the kinetic term we also need to write down the oscillator form of $Q$,

$$
Q=\sum_{n \in \mathbb{Z}} c_{n}\left(L_{-n}^{m}-\delta_{n, 0}\right)+\sum_{m, n \in \mathbb{Z}} \frac{m-n}{2}: c_{m} c_{n} b_{-m-n}:
$$

where we indicated that the second term is normal ordered. The matter Virasoro operators $L_{n}^{m}$ appearing in (2.39) are obtained from expending the energy momentum tensor $T^{m}$ (1.22). Explicitly, the relevant ones at $l_{0}=1$ are,

$$
\begin{aligned}
L_{0} & =\sum_{n=1}^{\infty} n a_{n}^{\dagger} a_{n}+\alpha^{\prime}\left(p^{2}+i p V\right), \\
L_{-1} & =\sqrt{2 \alpha^{\prime}} p a_{1}^{\dagger}+\sum_{n=1}^{\infty} \sqrt{n(n+1)} a_{n+1}^{\dagger} a_{n}, \\
L_{1} & =\sqrt{2 \alpha^{\prime}}(p+i V) a_{1}+\sum_{n=1}^{\infty} \sqrt{n(n+1)} a_{n}^{\dagger} a_{n+1} .
\end{aligned}
$$

For the evaluation of the cubic term we need to know the three-vertex, which is unfortunately more complicated than the two-vertex.

$$
\begin{aligned}
{ }_{123}\left\langle V_{3}^{m}\right|= & \mathcal{N} \int d^{d} p_{1} d^{d} p_{2} d^{d} p_{3}{ }_{1}\left\langle\left. p_{1}\right|_{2}\left\langle p_{2}\right|{ }_{3}\left\langle p_{3}\right| \delta^{d}\left(p_{1}+p_{2}+p_{3}+i V\right)\right. \\
& \cdot \exp \left(-\sum_{r, s=1}^{3}\left(\sum_{n, m=1}^{\infty} \frac{1}{2} a_{n}^{r} V_{n m}^{r s} a_{m}^{s}+\sum_{n=1}^{\infty} a_{n}^{r} V_{n 0}^{r s} p_{s}+\frac{1}{2} p_{r} V_{00}^{r s} p_{s}\right)\right) \\
{ }_{123}\left\langle V_{3}^{g}\right|= & { }_{123}\langle\Omega| c_{0}^{3} c_{0}^{2} c_{0}^{1} \exp \left(\sum_{r, s=1}^{3} \sum_{m=0}^{\infty} \sum_{n=1}^{\infty} b_{m}^{r} X_{m n}^{r s} c_{n}^{s}\right)
\end{aligned}
$$

Here, we suppressed the index $\mu$, on which the oscillators and some of the coefficients depend, for clarity. The $V_{n m}^{r s}$ and $X_{m n}^{r s}$ coefficients are independent of the linear dilaton. 
They are found, e.g. in [3],

$$
\begin{aligned}
V_{n m}^{r s} & =-\frac{1}{\sqrt{n m}} \oint \frac{d w}{2 \pi i} \oint \frac{d z}{2 \pi i} \frac{1}{z^{m} w^{n}} \frac{f_{r}^{\prime}(z) f_{s}^{\prime}(w)}{\left(f_{r}(z)-f_{s}(w)\right)^{2}} \\
X_{m n}^{r s} & =\oint \frac{d w}{2 \pi i} \oint \frac{d z}{2 \pi i} \frac{1}{z^{n-1} w^{m+2}} \frac{f_{s}^{\prime}(z)^{2}}{f_{r}^{\prime}(w)\left(f_{s}(z)-f_{r}(w)\right)} \prod_{I=1}^{3} \frac{f_{r}(w)-f_{I}(0)}{f_{s}(z)-f_{I}(0)}
\end{aligned}
$$

where $f_{r}$ are the conformal transformations defining the three-vertex (2.12)-(2.15). The normalization factor $\mathcal{N}$ and the momentum dependence can be read by comparing to the expressions obtained for the tachyon using CFT methods. The result is

$$
\begin{aligned}
\mathcal{N} & =K^{3-\alpha^{\prime} V^{2}}, \\
V_{00}^{r s} & =2 \alpha^{\prime} \log K \delta^{r s} .
\end{aligned}
$$

For evaluating $V_{n 0}^{r s}$ we again compare the expressions obtained using oscillator methods and CFT methods. The oscillator representation is,

$$
{ }_{123}\left\langle V_{3}^{m}\left|a_{n}^{\dagger 1}\right| p_{1}\right\rangle\left|p_{2}\right\rangle\left|p_{3}\right\rangle=-\sum_{s=1}^{3} V_{n 0}^{1 s} p_{s}\langle\text { tachyon }\rangle,
$$

where the value of the expression without the $a^{\dagger}$ insertion, which equals the expectation value for three tachyon interaction is written as $\langle$ tachyon $\rangle$. On the CFT side we obtain,

$$
\begin{aligned}
{ }_{123} & \left\langle V_{3}^{m}\left|a_{n}^{\dagger r}\right| p_{1}\right\rangle\left|p_{2}\right\rangle\left|p_{3}\right\rangle \\
& =\oint \frac{d z}{2 \pi i} \sqrt{\frac{2}{\alpha^{\prime}}} \frac{1}{\sqrt{n} z^{n}}\left\langle f_{1} \circ(i \partial X)(z) f_{1} \circ e^{i p_{1} X}(0) f_{2} \circ e^{i p_{2} X}(0) f_{3} \circ e^{i p_{3} X}(0)\right\rangle \\
& =\oint \frac{d z}{2 \pi i} \sqrt{\frac{2}{\alpha^{\prime}}} \frac{\alpha^{\prime}}{\sqrt{n} z^{n}}\left(\sum_{s=1}^{3} \frac{f_{1}^{\prime}(z) p_{s}}{f_{1}(z)-f_{s}(0)}+\frac{i V}{2} \frac{f_{1}^{\prime \prime}(z)}{f_{1}^{\prime}(z)}\right)\langle\text { tachyon }\rangle \\
& =\oint \frac{d z}{2 \pi i z^{n}} \sqrt{\frac{2 \alpha^{\prime}}{n}} \sum_{s=1}^{3} p_{s}\left(\frac{f_{1}^{\prime}(z)}{f_{1}(z)-f_{s}(0)}-\frac{f_{1}^{\prime \prime}(z)}{2 f_{1}^{\prime}(z)}\right)\langle\text { tachyon }\rangle .
\end{aligned}
$$

Here, we used the CFT definition of the expression and (1.12) in the first equality. Then, we used the non-tensor transformation rule (1.29) in the second equality and the anomalous momentum conservation in the last equality. We can now infer,

$$
V_{n 0}^{1 s}=-\oint \frac{d z}{2 \pi i z^{n}} \sqrt{\frac{2 \alpha^{\prime}}{n}}\left(\frac{f_{1}^{\prime}(z)}{f_{1}(z)-f_{s}(0)}-\frac{f_{1}^{\prime \prime}(z)}{2 f_{1}^{\prime}(z)}\right) .
$$

Note, that there is no lose of generality from choosing $r=1$, due to the cyclicity property of the three-vertex,

$$
V^{r s}=V^{(r+n)(s+n)} \quad \forall n,
$$

where the indices are added modulo 3. Also note, that the expression we obtained does not agree with the literature even in the limit $V \rightarrow 0$ (which is a trivial limit, since the 
final expression is $V$-independent). The reason is that without a linear dilaton $V_{n 0}^{r s}$ is only defined up to $V_{n 0}^{r s} \rightarrow V_{n 0}^{r s}+K_{n}^{s}$, for arbitrary constants $K_{n}^{s}$, due to the non-anomalous momentum conservation. This freedom is used, e.g. in $[85,86]$ in order to set $\sum_{s} V_{n 0}^{r s}=0$. If we do not wish to have a term proportional to $i V a^{\dagger}$ in the definition of the three-vertex, then we have no redefinition freedom and we are forced to use (2.48). One can verify that this result indeed makes sense, by noticing that, unlike other expressions for the threevertex, it is SL(2) invariant. We are almost ready now to address higher levels. The only issue that should still be clarified is the form of the reality condition, to which we turn next.

\subsection{The reality condition}

Let us recall the reality condition of the string field. This condition states that the string field is left invariant under the combined action of two involutions, Hermitian conjugation $\mathcal{O} \rightarrow \mathcal{O}^{\dagger}$ and $\mathrm{BPZ}$ conjugation $\mathcal{O} \rightarrow \mathcal{O}^{b}$. The former is the more familiar one. It sends $|0\rangle$ to $\langle 0|, \mathcal{O}_{n}$ to $\mathcal{O}_{-n}$, where $\mathcal{O}$ stands for either $a, b$ or $c$, while reversing the order of operators. It also induces complex conjugation. BPZ conjugation is performed by the action of the two-vertex $\left\langle V_{2}\right|$ (2.38). It also sends $|0\rangle$ to $\langle 0|$. However, it does not induce complex conjugation, nor does it change the order of operators. It also acts differently on the various operators,

$$
a_{n}^{b}=(-1)^{n+1} a_{-n}, \quad c_{n}^{b}=(-1)^{n+1} c_{-n}, \quad b_{n}^{b}=(-1)^{n} b_{-n} .
$$

The different signs originate from the odd conformal dimension of $\partial X(z)$ and $c(z)$ versus the even one of $b(z)$. Combining the two involutions leaves us with $(-1)^{\# a_{\text {even }}+\# c_{\text {even }}+\# b_{\text {odd }}}$ times the original operators inversely ordered. It is important to note that the coefficient fields also change their order relative to the other expressions. This is important when the Grassmann odd coefficient fields of even-ghost-number string fields are considered. The coefficient fields are also complex conjugated. We prefer to work with coefficient fields which are defined to be real. Thus, matching the signs translates into the choice of putting an extra $i$ factor in front of some of the coefficients. Note, that we do not have to separate the $c_{1}$ factor from the vacuum $|\Omega\rangle$, since it does not contribute a sign and also commutes with the rest of the operators, which are Grassmann even when coefficient fields are included.

We write the action in terms of momentum modes. The rules for settling the reality of the component fields, when applied to the explicit momentum dependence lead to the (almost) standard reality in momentum space,

$$
\hat{\phi}(p)=\hat{\phi}(-p-i V)^{*},
$$

where $\hat{\phi}$ is an arbitrary component field and $p$ is the momentum. To get from this expression a genuine standard reality condition we have to impose the same transformation that we imposed in (2.23),

$$
\hat{\phi}(p)=\phi\left(p+i \frac{V}{2}\right) .
$$

With this definition the reality condition takes the familiar form,

$$
\phi(p)=\phi(-p)^{*} \Longleftrightarrow \phi(x)=\phi(x)^{*} .
$$


We would like to work from now on only with the real fields. This can be achieved by redefining $Q$, the matter two-vertex $\left\langle V_{2}^{m}\right|$ (2.38a) and the matter three-vertex $\left\langle V_{3}^{m}\right|$ (2.41a) in a way that compensates for the transformation (2.52). The redefinition of $\left|V_{2}\right\rangle$ is nothing but the replacement,

$$
\delta^{d}(p+q+i V) \rightarrow \delta^{d}(p+q),
$$

in (2.38a). The redefined $Q$ is the same as the old one, only with the matter Virasoro operators redefined from (2.40) to the more symmetric form,

$$
\begin{aligned}
L_{0} & =\sum_{n=1}^{\infty} n a_{n}^{\dagger} a_{n}+\alpha^{\prime}\left(p^{2}+\frac{V^{2}}{4}\right), \\
L_{-1} & =\sqrt{2 \alpha^{\prime}}\left(p-i \frac{V}{2}\right) a_{1}^{\dagger}+\sum_{n=1}^{\infty} \sqrt{n(n+1)} a_{n+1}^{\dagger} a_{n}, \\
L_{1} & =\sqrt{2 \alpha^{\prime}}\left(p+i \frac{V}{2}\right) a_{1}+\sum_{n=1}^{\infty} \sqrt{n(n+1)} a_{n}^{\dagger} a_{n+1},
\end{aligned}
$$

and similarly for the other modes. We see that not only the string fields, but also the Virasoro modes obey now the standard reality condition,

$$
L_{n}^{\dagger}=L_{-n} .
$$

It is straightforward to see that with the new definition one recovers (2.24).

Transforming the cubic interaction according to (2.52) is nothing but the replacement of $p_{r}$ by $p_{r}-\frac{i V}{2}$ everywhere in the three vertex. This results in,

$$
\begin{aligned}
\delta^{d}\left(p_{1}+p_{2}+p_{3}+i V\right) & \rightarrow \delta^{d}\left(p_{1}+p_{2}+p_{3}-\frac{i V}{2}\right), \\
\mathcal{N} & \rightarrow K^{3\left(1-\frac{\alpha^{\prime} V^{2}}{4}\right)} \\
V_{n 0}^{1 s} & \rightarrow V_{n 0}^{1 s}-\sum_{r=1}^{3} V_{n 0}^{1 r} .
\end{aligned}
$$

\subsection{Truncation of the action to $l_{0}=1$ in scheme 4}

We now have all the ingredients needed for defining the $l_{0}=1$ action, which we evaluate for a general dimension $d$. The $l_{0}=1$ component of the string field can be written in terms of six real component fields,

$$
\begin{aligned}
\Psi_{1}=\int d^{d} p( & A_{\mu}(p) a_{1}^{\mu \dagger}+B(p) b_{-1}+i C(p) c_{-1}+ \\
& \left.\left(\mathcal{A}_{\mu}(p) a_{1}^{\mu \dagger}+i \mathcal{B}(p) b_{-1}+\mathcal{C}(p) c_{-1}\right) c_{0}\right) e^{i p \cdot X}|\Omega\rangle .
\end{aligned}
$$

Of the new six fields, the second line includes the ones which are outside the Siegel gauge. These fields do not contribute to our schemes 2 and 4 . The only fields with ghost number one are the "photon" $A$ and the auxiliary field $\mathcal{B}$. These are the fields that contribute to 
scheme number 1 . Of these, only the photon contributes to scheme 2. Scheme 4 carries all the fields of the first line, while scheme 3 carries not only all the new fields, but also the field $\mathcal{T}$, from $l_{0}=0$, which did not contribute to the action previously. It is important to remember that the fields $B, C, \mathcal{T}$ and $\mathcal{A}$ are Grassmann odd fields.

We now want to evaluate the kinetic term of the new fields. Since we assume that the fields in $\Psi_{1}$ are real, we should work with the modified $\left\langle V_{2}\right|(2.54)$ and $L_{n}$ (2.55). At this level the BRST charge $Q$ is truncated to

$$
Q=c_{0}\left(L_{0}^{m}-1\right)+c_{1} L_{-1}^{m}+c_{-1} L_{1}^{m}-b_{-1} c_{0} c_{1}-c_{-1} c_{0} b_{1}+2 c_{-1} b_{0} c_{1} .
$$

Assume for now that we work with scheme 4. Then, we have the even fields $T$ and $A$ and the odd fields $B$ and $C$. Note, that due to our treatment of the reality condition, $T$ now is what we called $\tau$ in section 2.1. The form of the BRST charge $Q$ can now be further simplified by disregarding all terms that do not include $c_{0}$,

$$
Q=c_{0}\left(L_{0}^{m}-1\right)-b_{-1} c_{0} c_{1}-c_{-1} c_{0} b_{1} .
$$

Direct evaluation now gives,

$$
S_{2}=-\int d^{d} x\left(\frac{m_{0}^{2} T^{2}+(\nabla T)^{2}}{2}+\frac{m_{1}^{2} A^{2}+\left(\partial_{\nu} A_{\mu}\right)^{2}}{2}+i\left(m_{1}^{2} B C+\nabla B \cdot \nabla C\right)\right) .
$$

Here, we used the generalization of (2.4),

$$
m_{l_{0}}^{2} \equiv \frac{V^{2}}{4}+\frac{l_{0}-1}{\alpha^{\prime}}
$$

for $l_{0}=1$. Note, that the kinetic term of the vector takes the standard form of a vector in the Feynman gauge.

We now turn to evaluating the cubic terms. Ghost number conservation dictates that the only possible interactions include $T^{3}, A T^{2}, A^{2} T, A^{3}, T B C$ and $A B C$. We have to evaluate all these terms. To that end we need the coefficients $V_{10}^{r s}, V_{11}^{r s}$ and $X_{11}^{r s}$. Using (2.48) we obtain,

$$
V_{10}^{11}=0, \quad V_{10}^{12}=\sqrt{\frac{8 \alpha^{\prime}}{27}}, \quad V_{10}^{13}=-\sqrt{\frac{8 \alpha^{\prime}}{27}} .
$$

These values should have been modified according to (2.59). However, they do not change, since they sum up to zero. In fact, this is the case for all odd values of $n$. For terms at least quadratic in $A$ we also have to use (2.42) for evaluating,

$$
V_{11}^{12}=V_{11}^{13}=-\frac{16}{27}
$$

while for the terms involving the ghost fields we need (2.43),

$$
X_{11}^{12}=X_{11}^{21}=-\frac{8}{27} .
$$

The evaluation of the $T^{3}$ term is straightforward and leads to the result already obtained (2.25),

$$
S_{3}^{1}=-\frac{g_{o} \mathcal{N}}{3} \int d^{d} x \tilde{T}^{3} e^{-\frac{V \cdot x}{2}} .
$$


Here and in what follows we leave the $x$ argument (of $\left.\tilde{T}(x)^{3}\right)$ implicit. Next, we get the $T^{2} A$ term,

$$
\begin{aligned}
S_{3}^{2} & =-\frac{g_{o}}{3} 3\left\langle V_{3}\right| \int d^{d} p_{1} d^{d} p_{2} d^{d} p_{3}\left(a_{1}^{\dagger} A\left(p_{1}\right)\left|p_{1}, \Omega\right\rangle\right)\left(T\left(p_{2}\right)\left|p_{2}, \Omega\right\rangle\right)\left(T\left(p_{3}\right)\left|p_{3}, \Omega\right\rangle\right) \\
& =-g_{o} \mathcal{N} \int d^{3 d} p \delta\left(\sum p_{i}-\frac{i V}{2}\right) \tilde{A}\left(p_{1}\right) \tilde{T}\left(p_{2}\right) \tilde{T}\left(p_{3}\right)\left(-V_{10}^{11} p_{1}-V_{10}^{12} p_{2}-V_{10}^{13} p_{3}\right) \\
& =0,
\end{aligned}
$$

where in the last equality we used the fact that we obtain in the integrand an expression which is anti-symmetric with respect to $p_{2} \leftrightarrow p_{3}$. The $T A^{2}$ term, for which we have to write the space-time indices explicitly, is

$$
\begin{aligned}
S_{3}^{3}= & -\frac{g_{o}}{3} 3\left\langle V_{3}\right| \int d^{3 d}\left(a_{1}^{\mu \dagger} A_{\mu}\left(p_{1}\right)\left|p_{1}, \Omega\right\rangle\right)\left(a_{1}^{\nu \dagger} A_{\nu}\left(p_{2}\right)\left|p_{2}, \Omega\right\rangle\right)\left(T\left(p_{3}\right)\left|p_{3}, \Omega\right\rangle\right) \\
= & -g_{o} \mathcal{N} \int d^{3 d} p \delta\left(\sum p_{i}-\frac{i V}{2}\right) \tilde{A}_{\mu}\left(p_{1}\right) \tilde{A}_{\nu}\left(p_{2}\right) \tilde{T}\left(p_{3}\right)\left(-V_{11}^{12} \eta^{\mu \nu}+\right. \\
& \left.\left(-V_{10}^{11} p_{1}-V_{10}^{12} p_{2}-V_{10}^{13} p_{3}\right)^{\mu}\left(-V_{10}^{11} p_{2}-V_{10}^{12} p_{3}-V_{10}^{13} p_{1}\right)^{\nu}\right) \\
= & -g_{o} \mathcal{N} \int d^{3 d} p \delta\left(\sum p_{i}-\frac{i V}{2}\right) \tilde{A}_{\mu}\left(p_{1}\right) \tilde{A}_{\nu}\left(p_{2}\right) \tilde{T}\left(p_{3}\right) . \\
& \cdot \frac{8}{27}\left(\alpha^{\prime}\left(p_{3}^{\mu} p_{1}^{\nu}+p_{3}^{\nu} p_{2}^{\mu}-\left(p_{3}^{\mu} p_{3}^{\nu}+p_{1}^{\mu} p_{2}^{\nu}\right)\right)+2 \eta^{\mu \nu}\right) \\
= & -\frac{8 g_{o} \mathcal{N}}{27} \int d^{d} x\left(2 \tilde{A}^{\mu} \tilde{A}_{\mu} \tilde{T}+\alpha^{\prime}\left(\tilde{A}_{\mu} \tilde{A}_{\nu} \partial^{\mu} \partial^{\nu} \tilde{T}+\partial^{\mu} \tilde{A}_{\nu} \partial^{\nu} \tilde{A}_{\mu} \tilde{T}-2 \tilde{A}_{\nu} \partial^{\nu} \tilde{A}_{\mu} \partial^{\mu} \tilde{T}\right)\right) e^{-\frac{V \cdot x}{2}} .
\end{aligned}
$$

Then, we evaluate the $A^{3}$ term,

$$
\begin{aligned}
S_{3}^{4}= & -\frac{g_{o}}{3}\left\langle V_{3}\right| \int d^{3 d}\left(a_{1}^{\dagger} A\left(p_{1}\right)\left|p_{1}, \Omega\right\rangle\right)\left(a_{1}^{\dagger} A\left(p_{2}\right)\left|p_{2}, \Omega\right\rangle\right)\left(a_{1}^{\dagger} A\left(p_{3}\right)\left|p_{3}, \Omega\right\rangle\right) \\
= & -\frac{g_{o} \mathcal{N}}{3} \int d^{3 d} p \delta\left(\sum p_{i}-\frac{i V}{2}\right)\left(V_{11}^{12} \sum_{r, s} V_{10}^{r s} p_{s}+\sum_{r, s, t}\left(V_{10}^{1 r} p_{r}\right)\left(V_{10}^{2 s} p_{s}\right)\left(V_{10}^{3 t} p_{t}\right)\right) . \\
& \cdot \tilde{A}\left(p_{1}\right) \tilde{A}\left(p_{2}\right) \tilde{A}\left(p_{3}\right) \\
= & 0 .
\end{aligned}
$$

Here, we should have paid attention to the Lorentz indices. The result, however, does not change by doing so. We can now notice that, in the expressions above, all terms with an odd number of (vector) $A$ fields vanish, as expected. Similarly, the $A B C$ term vanishes. The calculation is the same as in (2.69), except that $T$ should be replaced by $B b_{-1}+i C c_{-1}$. Hence, we are left with the evaluation of the $T B C$ term,

$$
\begin{aligned}
S_{3}^{5}= & -\frac{g_{o}}{3} 3 i\left\langle V_{3}\right| \int d^{3 d}\left(\tilde{T}\left(p_{1}\right)\left|p_{1}, \Omega\right\rangle\right)\left(\left(\tilde{B}\left(p_{2}\right) b_{-1}\left|p_{2}, \Omega\right\rangle\right)\left(\tilde{C}\left(p_{3}\right) c_{-1}\left|p_{3}, \Omega\right\rangle\right)\right. \\
& \left.+\left(\tilde{C}\left(p_{2}\right) c_{-1}\left|p_{2}, \Omega\right\rangle\right)\left(\tilde{B}\left(p_{3}\right) b_{-1}\left|p_{3}, \Omega\right\rangle\right)\right) \\
= & -\frac{16 i g_{o} \mathcal{N}}{27} \int d^{d} x \tilde{B} \tilde{C} \tilde{T} e^{-\frac{V \cdot x}{2}} .
\end{aligned}
$$

The complete action up to $l_{0}=1$ (for scheme 4) is the sum of $S_{2}(2.63)$ and $S_{3}(2.68),(2.70)$ and (2.72). 


\subsection{Automatization using conservation laws}

So far we considered scheme 4 at $l_{0}=1$. If we remain at $l_{0}=1$, but switch to scheme 3 , we already have 8 component fields. This results in over a hundred possible interaction terms. While many of those trivially vanish in light of, e.g. ghost number conservation, many others have to be explicitly evaluated. Furthermore, the number of terms grows fast as we increase the level $l_{0}$, which is essential in order to obtain reliable results. Explicit evaluation of all terms would soon become hopeless. The resolution of this difficulty is to automate the evaluation of the various coefficients that appear in the action. The quadratic terms are easily calculated. For the evaluation of the cubic terms, an efficient method should be used. As in previous works that used level-truncation, we find that the most efficient method for the evaluation of these terms is using conservation laws of the cubic vertex [87].

Conservation laws are obtained by evaluating the expectation values of currents in the geometry of the three-vertex. These currents are built from products of the conformal fields, for which we want to derive the conservation laws, by conformal tensors of functions. The weight of these conformal tensors is properly chosen in order to obtain a current, and the functions are constrained in order to prevent singularities at any point other than the punctures, including infinity. Closing such a current around the three punctures leads to a linear combination of modes of the current, while deforming the current to infinity leads to zero, as long as the functions were properly constrained. Actually, some more terms can be obtained, both at infinity and around the punctures, if the current is anomalous, as is often the case (e.g. Virasoro operators in the case of a non-zero central charge $c$, ghost current, and $\partial X$ in the case of a linear dilaton system). However, these terms are also explicitly derived in [87].

Here, we need the conservation laws for the $b$ and $c$ ghosts and for the $\partial X$ (matter) modes. The lowest order conservation laws are,

$$
\begin{aligned}
\left\langle V_{3}\right| c_{0}^{2} & =\left\langle V_{3}\right|\left(\frac{4}{3 \sqrt{3}}\left(c_{1}^{1}-c_{1}^{3}\right)+\ldots\right), \\
\left\langle V_{3}\right| c_{-1}^{2} & =\left\langle V_{3}\right|\left(\frac{1}{27}\left(8 c_{1}^{1}+8 c_{1}^{3}+11 c_{1}^{2}\right)+\ldots\right), \\
\left\langle V_{3}\right| b_{-1}^{2} & =\left\langle V_{3}\right|\left(\frac{4}{3 \sqrt{3}}\left(b_{0}^{1}-b_{0}^{3}\right)-\frac{1}{27}\left(8 b_{1}^{1}+8 b_{1}^{3}+11 b_{1}^{2}\right)+\ldots\right), \\
\left\langle V_{3}\right| a_{-1}^{2} & =\left\langle V_{3}\right|\left(\sqrt{\frac{8 \alpha^{\prime}}{27}}\left(p_{3}-p_{1}\right)+\frac{1}{27}\left(16 a_{1}^{1}+16 a_{1}^{3}-5 a_{1}^{2}\right)+\ldots\right),
\end{aligned}
$$

where again, the superscript refers to the space in which the mode is defined and the ellipses indicate higher level modes. Note, that the matter conservation law includes the momentum explicitly. In principle, the dilaton slope $V$ could also occur. However, we can always eliminate it in favour of the momenta using the anomalous momentum conservation (2.57). The result then holds in any dimension. It might differ from the familiar flat space expressions by terms proportional to $p_{1}+p_{2}+p_{3}$. 


\subsection{The problem with scheme 3}

Since conservation laws are given in the momentum representation, it is easier to write down the action in this representation. For now we consider the one-dimensional case at $l_{0}=1$ in scheme 3 . Hence, Lorentz indices, when they appear, can obtain only a single value and are therefore omitted. The quadratic term is given by

$$
\begin{aligned}
S_{2}^{(3)}=-\int d p & \frac{m_{0}^{2}+p^{2}}{2} T(p) T(-p)+\frac{m_{1}^{2}+p^{2}}{2}(A(p) A(-p)+2 i B(p) C(-p)) \\
& \left.+\frac{\mathcal{B}(p)\left(\mathcal{B}(-p)+\sqrt{\frac{\alpha^{\prime}}{2}} V A(-p)\right)}{\alpha^{\prime}}-\frac{i V}{\sqrt{2 \alpha^{\prime}}} B(p) \mathcal{A}(-p)\right) .
\end{aligned}
$$

Here, the first line is the expression that we had before and the second line includes the new fields. It is seen that all these fields are auxiliary fields, since there are no new kinetic terms. Reality of the action is a consequence of the fact that products of even fields carry real coefficients, while products of odd fields carry imaginary coefficients.

For the evaluation of the cubic terms we use the conservation rules, which reduce the general cubic term to that of the elementary tachyon vertex

$$
\left\langle V_{3}|| \Psi_{1}\right\rangle_{1}\left|\Psi_{2}\right\rangle_{2}\left|\Psi_{3}\right\rangle_{3} \propto\left\langle V_{3}|| \Omega, p_{1}\right\rangle_{1}\left|\Omega, p_{2}\right\rangle_{2}\left|\Omega, p_{3}\right\rangle_{3} .
$$

The conservation laws give the proportionality coefficients, which can be zero and are momentum-dependent. We already evaluated the fundamental (three tachyon) term,

$$
\begin{aligned}
S_{3}^{(T T T)} & =-\frac{g_{o}}{3}\left\langle V_{3}|| \Omega\right\rangle_{1}|\Omega\rangle_{2}|\Omega\rangle_{3} \\
& =-\frac{g_{o} \mathcal{N}}{3} \int d p_{1} d p_{2} d p_{3} \delta\left(\sum_{j=1}^{3} p_{j}-\frac{i V}{2}\right) K^{-\alpha^{\prime} \sum_{k=1}^{3} p_{k}^{2}} .
\end{aligned}
$$

Here, we wrote $|\Omega\rangle_{k}$ instead of $\left|p_{k}, \Omega\right\rangle_{k}$ for short. Also, recall that the coefficient $\mathcal{N}$ is given by $(2.58)$.

Even before the use of the conservation laws there are several terms that can be discarded due to ghost number. The total ghost number of any three coefficient fields should equal three. In our treatment, where we build the states over the ghost number one $|\Omega\rangle$ vacuum, it means that the total ghost number other than that of the vacua should equal zero. From the correlation between ghost number and statistics of the component fields we can also infer that odd fields either do not appear, or appear as a pair, as should be the case for obtaining an even action. That means that we would be able to continue integrating those fields out, before commencing the simulations. All in all, there are only 19 possible terms that have to be evaluated.

In the evaluation of $S_{3}$ there are six contributions to a generic coefficient, which come from the six possible orderings of the three coefficient fields involved. The properties of the three-vertex implies that these coefficients can only depend on the cyclic order of the fields. Hence, the term in the action that involves the component fields $\Psi_{1} \Psi_{2} \Psi_{3}$ is given by,

$$
-g_{o}\left\langleV _ { 3 } \left|\left(\left|\Psi_{1}\right\rangle_{1}\left|\Psi_{2}\right\rangle_{2}\left|\Psi_{3}\right\rangle_{3}+\left|\Psi_{3}\right\rangle_{1}\left|\Psi_{2}\right\rangle_{2}\left|\Psi_{1}\right\rangle_{3}\right)\right.\right. \text {. }
$$


It turns out that in several cases the two orderings produce expressions that cancel out, after relabeling the three spaces, in particular, due to the momentum dependence of the result. Another issue, which we have to notice, is that of symmetry factors, i.e. if two component fields are the same, e.g. $\Psi_{1}=\Psi_{2}$, the result should be divided by two, while in the case $\Psi_{1}=\Psi_{2}=\Psi_{3}$, the result should be divided by six. Even better (computationally) is to divide the result by one and by three respectively, but to evaluate only one of the terms in (2.80), since in these cases there is no issue of different orderings.

We are now ready to write down the full expression in terms of (2.79),

$$
\begin{aligned}
S_{3}^{(3)}= & -\frac{g_{o} \mathcal{N}}{3} \int d p_{1} d p_{2} d p_{3} \delta\left(\sum_{j=1}^{3} p_{j}-\frac{i V}{2}\right) K^{-\alpha^{\prime} \sum_{k=1}^{3} p_{k}^{2}}\left(T\left(p_{1}\right) T\left(p_{2}\right) T\left(p_{3}\right)\right. \\
& +\frac{8}{9} A\left(p_{1}\right) A\left(p_{2}\right) T\left(p_{3}\right)\left(2-\alpha^{\prime}\left(p_{3}-p_{2}\right)\left(p_{3}-p_{1}\right)\right)+\frac{16 i}{9} T\left(p_{1}\right) B\left(p_{2}\right) C\left(p_{3}\right) \\
& -\frac{16}{9} T\left(p_{1}\right) \mathcal{B}\left(p_{2}\right) \mathcal{B}\left(p_{3}\right) \\
& +\frac{16 \sqrt{2 \alpha^{\prime}}}{9} B\left(p_{1}\right)\left(\mathcal{A}\left(p_{2}\right) T\left(p_{3}\right)-A\left(p_{2}\right) \mathcal{T}\left(p_{3}\right)\right)\left(p_{3}-p_{1}\right) \\
& \left.+\frac{32 i}{9} B\left(p_{1}\right) \mathcal{B}\left(p_{2}\right) \mathcal{T}\left(p_{3}\right)\right) .
\end{aligned}
$$

Here, the first two lines are the expression that we had for scheme 4, the third line includes a new bosonic interaction and the last two lines include two new interaction terms involving odd fields.

The path integral (2.33) now contains also integration over the various new modes. In particular one expects it to contain integration over the odd variables included, namely, $B$, $C, \mathcal{T}$ and $\mathcal{A}$

$$
Z=\int\left(\prod_{j} d T_{j} d \mathcal{T}_{j}\right)\left(\prod_{n} d \mathcal{B}_{n} d B_{n} d \mathcal{C}_{n} d C_{n} d A_{n} d \mathcal{A}_{n}\right) e^{S}
$$

Here and in the rest of the paper $T_{j}$ (denoted $\tau_{n}$ above), $\mathcal{T}_{j}, A_{n}$, etc., represent the modes of the various fields. The fields appear in the measure in pairs of an even and an odd field, with the even ones written first. We need two different indices for the products since the number of modes of a given field depends on its $l_{0}$. We would also like, if possible, to integrate out the bosonic auxiliary field $\mathcal{B}$. Since at higher levels it would be quite impossible to eliminate all the auxiliary fields, it could be nice to compare the results with and without the elimination of $\mathcal{B}$. The auxiliary bosonic field $\mathcal{C}$ does not appear in the action at all.

Inspecting the action (2.77) and (2.81) we recognize that it suffers from a major problem: a Grassmann integral can be non-zero only if the integrand has a term, which is linear with respect to all the Grassmann modes. However, a term linear in all the odd fields is absent in the path integral. Since odd terms enter the various terms in the action either quadratically or not at all, the problem of saturating all the modes is that of the regularity of the (bosonic-field-dependent) matrix of coefficients of the terms quadratic with respect to the odd variables in the action. The problem then is that this matrix turns out to be singular. 
This problem occurs since level truncation does not commute with Grassmann integration. Actually, we faced this problem already at level zero, where we noticed that the field $\mathcal{T}$, which is present in (2.2) is absent from the action altogether. There, we decided to ignore this field temporarily and it indeed enters the action now. However, it is not clear which fields should we retain now and which ones should be postponed to the next level. Inspecting the action we see that the field $B$ is present in all the relevant expressions and is saturated in each term by one of the other fermionic fields, namely $\mathcal{T}, C$ and $\mathcal{A}$. This is not particularly surprising, due to the ghost number of the states that these component fields multiply. However, it is not clear which modes should we keep now. The most "natural" choice would be to keep $\mathcal{T}$, since it already "enters too late to the game". However, one could object to the idea of adding high $\mathcal{T}$ modes before adding the first $C$ modes, since it would make our cutoff $l_{0}$-dependent instead of $l$-dependent. Furthermore, since the modes of $\mathcal{T}$ and $B$ enter the level truncation at different cut-off values, we would generally have a different number of such modes and it would be impossible to saturate the Grassmann integral.

One could worry that such problems could occur also for scheme 4, which also includes odd modes. This is not the case. The source of the problem we face here is the fact that the fields $\mathcal{T}$ and $\mathcal{A}$ are auxiliary and hence do not have kinetic terms. The kinetic terms provide regular parts for the matrix. Hence, the integral over the odd fields is regular for scheme 4, except perhaps for some specific values for the bosonic fields.

An additional potential difficulty with scheme 3, is that it is likely to inherit from scheme 1 the problem, to be described in 4.9, of a nearly-massless mode leading to large instabilities. In light of all that we do not dwell further into scheme 3.

\subsection{The action in scheme 1}

We also would like to check scheme 1 , in which we only keep the fields $T, A$ and $\mathcal{B}$. The action is just the truncation of the scheme- 3 action to include only these fields. The quadratic part of the action is

$$
\begin{aligned}
S_{2}^{(1)}=-\int d p & \frac{m_{0}^{2}+p^{2}}{2} T(p) T(-p)+\frac{m_{1}^{2}+p^{2}}{2} A(p) A(-p) \\
+ & \left.\frac{\mathcal{B}(p)\left(\mathcal{B}(-p)+\sqrt{\frac{\alpha^{\prime}}{2}} V A(-p)\right)}{\alpha^{\prime}}\right)
\end{aligned}
$$

and the cubic part is

$$
\begin{aligned}
S_{3}^{(1)}= & -\frac{g_{o} \mathcal{N}}{3} \int d p_{1} d p_{2} d p_{3} \delta\left(\sum_{j=1}^{3} p_{j}-\frac{i V}{2}\right) K^{-\alpha^{\prime} \sum_{k=1}^{3} p_{k}^{2}}\left(T\left(p_{1}\right) T\left(p_{2}\right) T\left(p_{3}\right)\right. \\
& +\frac{8}{9} A\left(p_{1}\right) A\left(p_{2}\right) T\left(p_{3}\right)\left(2-\alpha^{\prime}\left(p_{3}-p_{2}\right)\left(p_{3}-p_{1}\right)\right) \\
& \left.-\frac{16}{9} T\left(p_{1}\right) \mathcal{B}\left(p_{2}\right) \mathcal{B}\left(p_{3}\right)\right) .
\end{aligned}
$$

The explicit integration of the $\mathcal{B}$ field should be much easier in this scheme as compared to schemes 3 and 4 . 


\subsection{Analytical study of the lowest mode}

Before we attempt a numerical study of the case with many modes, we would like to examine analytically the simplest possibility of retaining a single mode. Hopefully, we could get some feeling about what should be expected from this simple example. The lowest lying mode would be the first mode of the tachyon field. Its level depends on the length $L$ of the range which we consider for $X$ and it is given by

$$
T(x)=\sqrt{\frac{2}{L}} \sin \left(\frac{\pi n\left(x-x_{\min }\right)}{L}\right) T,
$$

where $T$ is the only variable in the theory. The action is

$$
S=-\frac{1}{2}\left(\frac{1}{24 \alpha^{\prime}}+\left(\frac{\pi}{L}\right)^{2}\right) T^{2}-\frac{\tilde{g}_{o} f}{3} K^{-\alpha^{\prime}\left(\frac{\pi}{L}\right)^{2}} T^{3},
$$

where we absorbed some constants into the coupling constant and the single coupling constant of the theory is found to be

$$
f=\left(\frac{2}{L}\right)^{\frac{3}{2}} \int_{-\frac{L}{2}}^{\frac{L}{2}} d x e^{-\frac{V x}{2}} \sin ^{3}\left(\frac{\pi\left(x-\frac{L}{2}\right)}{L}\right)=\left(\frac{2}{L}\right)^{\frac{3}{2}} \frac{192 \pi^{3} L \cosh \left(\frac{L V}{4}\right)}{L^{4} V^{4}+40 \pi^{2} L^{2} V^{2}+144 \pi^{4}} .
$$

Here for simplicity we take the range of integration to be symmetric with respect to the origin. It is easy to see that, as one should expect, $f$ approaches infinity as $L \rightarrow \infty$.

Performing the advocated analytical continuation $T \rightarrow e^{i \pi / 6} T$ (see section 3 for details) the action becomes

$$
S=-\frac{e^{\frac{i \pi}{3}}}{2}\left(\frac{1}{24 \alpha^{\prime}}+\left(\frac{\pi}{L}\right)^{2}\right) T^{2}-\frac{i \tilde{g}_{o} f}{3} K^{-\alpha^{\prime}\left(\frac{\pi}{L}\right)^{2}} T^{3} .
$$

The simplicity of this expression makes it possible to evaluate the partition function analytically. Write,

$$
S=-a(L) T^{2}-i b(L, V) T^{3} .
$$

Then, the partition function is given by,

$$
\begin{aligned}
Z & =\int_{-\infty}^{\infty} d T e^{S}=\int_{-\infty}^{\infty} d T e^{-a T^{2}-i b T^{3}}=b^{-\frac{1}{3}} \int_{-\infty}^{\infty} d T e^{-a b^{-\frac{2}{3}} T^{2}-i T^{3}} \\
& =\frac{2 \pi e^{\frac{2 a^{3}}{27 b^{2}}}}{(3 b)^{\frac{1}{3}}} \operatorname{Ai}\left(\frac{a^{2}}{(3 b)^{4 / 3}}\right),
\end{aligned}
$$

where $\mathrm{Ai}$ is the Airy function. We know that the integral converges, since our $a$ has a positive real part. However, the result is not real, as expected. Nonetheless, when we take the limit $L \rightarrow \infty$, the partition function approaches a real value. In this limit (setting $\alpha^{\prime}=1$ ) we have (regardless of the value of $V$ ),

$$
a \rightarrow \frac{e^{\frac{i \pi}{3}}}{48}, \quad b \rightarrow \infty,
$$

where the approach of $b$ to infinity is along the positive real line. The factor in front of the Airy function is real and approaches zero as $L \rightarrow \infty$. The Airy function, on the other hand, is complex. However, it has a real limit,

$$
\operatorname{Ai}\left(\frac{a^{2}}{(3 b)^{4 / 3}}\right) \rightarrow \frac{1}{3^{\frac{2}{3}} \Gamma\left(\frac{2}{3}\right)} \text {. }
$$


In principle, we were not supposed to expect a real limit here, since we are truncating to the lowest single mode. Nonetheless, it is encouraging to see that the wild oscillations conspire to produce a real value already at this stage. Also, we see that reality is really obtained only as we take the limit $L \rightarrow \infty$. Thus, comparing finite values does not necessarily make sense.

Using (2.90), we can study the dependence of various expectation values as a function of $a$ and $b$. One can obtain different values for these coefficients in many ways, by using symmetric as well as non-symmetric limits for $x_{\min }$ and $x_{\max }$. The limit $b \rightarrow 0$ gives a free theory, in which $\langle S\rangle=-\frac{1}{2}$, while $\langle T\rangle$ approaches zero from the direction of $e^{\frac{7 i \pi}{6}}$ and $\left\langle T^{2}\right\rangle$ approaches zero from the direction $e^{-\frac{i \pi}{3}}$. Conversely, in the mentioned above limit $b \rightarrow \infty$, we find that $\langle S\rangle=-\frac{1}{3}$, while $\langle T\rangle$ approaches zero from the direction $e^{-\frac{i \pi}{6}}$ and $\left\langle T^{2}\right\rangle$ approaches zero from the direction $e^{\frac{i \pi}{3}}$. We will compare these results to the lattice simulation of section $4 .{ }^{11}$

Another important remark regarding the fact that the normalization factor approaches zero: on the one hand, the normalization factor should be renormalized as we change our parameters. Thus, from this perspective, there is nothing here to discuss. On the other hand, keeping only the lowest level amounts to truncating more and more modes as $L$ approaches infinity. This is not a natural limit and we took it only for the purpose of verifying that we can reproduce on the lattice the analytical expression that we obtain using it. The natural limit that we would have to consider is taking $L$ to infinity while keeping $l$ fixed. This leads to more and more modes, up to infinity at the limit. This is the physical limit. However, the increase in the parameters, as well as the introduction of an ever growing number of modes, imply that a non-trivial renormalization would be needed.

\subsection{Adding trivial terms to the action}

Another problem that a lattice simulation in a linear dilaton background faces comes from the possible presence in the action of trivial terms. By that we mean the presence in the definition of the cubic interaction of terms that vanish due to the anomalous momentum conservation. Such terms can be added to the definition of the vertex also in the standard case of a constant dilaton. In any case they take the form of conformal fields inserted at the three interaction points times a momentum dependent function of the form

$$
F\left(p_{1}, p_{2}, p_{3}\right)=p_{1}^{n_{1}} p_{2}^{n_{2}} p_{3}^{n_{3}}\left(p_{1}+p_{2}+p_{3}-\frac{i V}{2}\right) \quad n_{1,2,3} \geq 0 .
$$

The expression in the brackets is identical to the argument of the momentum conservation delta function and thus leads to zero contribution of these terms, which can, therefore, be added to the definition of the interaction at will. In previous works use was made of such terms in order to simplify the form of the interaction in various contexts, e.g. in [86].

While the ambiguity in these terms is usually harmless and could even be useful, in our case new complications emerge. The momentum conservation is broken by the introduction

\footnotetext{
${ }^{11}$ Note, that in section 4 we use a slightly different convention, in which the factor of $e^{\frac{i \pi}{6}}$ explicitly multiplies the fields. This leads to different constant phases as compared to what we did here.
} 
of the lattice. Thus, while the introduction of these terms does not change the action before the introduction of the lattice, it does influence the results when a lattice is used. A simple idea for a resolution would be to avoid these terms altogether. However, it is not clear how to distinguish the "genuine" interaction from the trivial terms. The definition of the action is really ambiguous. This is somewhat similar to the case of a gauge symmetry: there is no canonical way to gauge fix. The analogue of gauge fixing in our case is the decision of which is the correct form of the action. However, on the lattice different "gauge fixings" lead to different results. One would like to be able to show that as the lattice cutoff is removed, the results tend to the same values regardless of the "gauge choice". Unfortunately, this seems to be quite unlikely, since the coefficients of the trivial terms can be arbitrary and more and more such terms pop up as the level is being increased. One could try to fix the ambiguity by demanding that the form of the interaction be "as simple as possible". While this statement makes sense at low levels, it becomes ambiguous at higher levels. Another possibility for a "gauge fixing" is to avoid the appearance of $V$ in the action other than in the exponent. While this option does not necessarily lead to the simplest possible expressions, as we have already seen in our $l_{0}=1$ example above, the expressions are unambiguous and are formally independent of the dimension $d$. Unfortunately, it is not clear that the expressions obtained in this way are more correct than those of any other "gauge choice".

It is important to stress that the problem could have been avoided had we been working in a constant dilaton background. In such a case we would have chosen to work with periodic boundary conditions that do not make sense in the case at hand. Then, momentum conservation would not have been broken by the lattice. Moreover, the presence of the linear dilaton leads to yet another problem due to the anomalous form of the conservation law. The issue is not so much the fact that the sum of momenta is non-zero, as with the fact that it is imaginary. This is not a problem before the introduction of the lattice, since it only results in the exponential term in coordinate space. However, with the introduction of the lattice actual imaginary terms pop-up. These terms produce further problems: as we mentioned above, the action being cubic is not bounded from below, a problem that we resolve using a change of the contour of integration followed by an analytical continuation. This procedure turns the real cubic terms to purely imaginary terms, which results in convergence of the expressions. However, the imaginary terms become real now, which brings us back to the starting point, in which no numerical analysis is possible. One could hope that the ambiguity in the form of the interaction term can be used in order to set to zero the imaginary part of the interaction. We examine the consequences of adding trivial parts to the action in section 4.8 .

\section{Lattice setup}

We now want to use lattice simulations to calculate observables. The degrees of freedom are the fields found above using level truncation, up to some maximum total level $l_{\max }$, not necessarily an integer. Explicitly, (1.5) can be written as

$$
l=l_{0}+\alpha^{\prime} p^{2} .
$$


For our sine-expansion, $p=\frac{\pi n}{L}$, and since we have only evaluated the level truncation up to $l_{0}=1$ we must choose $l<2$. The number of modes for the $l_{0}=0$ fields is then

$$
n_{0}=\left\lfloor\frac{L}{\pi} \sqrt{\left.\frac{l_{\max }}{\alpha^{\prime}}\right\rfloor}\right.
$$

and if $l_{\max }>1$ the number of modes for the $l_{0}=1$ fields is

$$
n_{1}=\left\lfloor\frac{L}{\pi} \sqrt{\frac{l_{\max }-1}{\alpha^{\prime}}}\right\rfloor .
$$

Thus given a lattice size $L$ and a choice of 'scheme', our degrees of freedom will be a finite number of modes of one or more fields. We can read off the action from the appropriate expressions above, e.g. for scheme 4 and $l<1$ we would need the terms $(2.30)$ and (2.31). We remind that the weight of a configuration in the path integral is $e^{S}$ rather than $e^{-S}$ due to the way we Wick-rotated. In addition to the various 'schemes' described above, we have also carried out additional runs where we have removed the level-1 fields from the action. This is to try to assess whether the higher level fields are helping to tame the instabilities.

Looking at the action we see an immediate problem: the action has a cubic instability. To proceed, we consider the integral over each mode as a complex integral, and deform the integration contour to be a straight line at an angle $\gamma$ to the real axis. If we choose $\gamma=\pi / 6$, the cubic part of the action becomes pure imaginary and so the action is no longer unstable. In principle we could have chosen different phases, i.e. $\gamma= \pm \pi / 6$ for different components of the string field. However, we refrain from doing so in order to treat the string field as a uniform physical entity. This is in accord with our strategy of using a single expression for the level (3.1), instead of considering separately $l_{0}$ and the momentum.

However, taking the modes to be complex introduces another problem; the action also becomes complex and so cannot be interpreted as a weight for a Markov chain. Instead we simulate in the phase-quenched ensemble and reweight. That is, we split $e^{S}$ into an amplitude and a phase:

$$
e^{S}=\left|e^{S}\right| e^{i \theta},
$$

and calculate the expectation value of an observable $\mathcal{O}$ using the identity

$$
\begin{aligned}
\langle\mathcal{O}\rangle & =\frac{\int \mathcal{O}\left|e^{S}\right| e^{i \theta}}{\int\left|e^{S}\right| e^{i \theta}} \\
& =\frac{\left\langle\mathcal{O} e^{i \theta}\right\rangle_{\mathrm{PQ}}}{\left\langle e^{i \theta}\right\rangle_{\mathrm{PQ}}}
\end{aligned}
$$

where the label PQ means the expectation value is evaluated in the phase-quenched ensemble, i.e. with the weight $\left|e^{S}\right|$. This is a real, positive weight, so it can be used in a Monte Carlo simulation.

We generate configurations in the phase-quenched ensemble using a Metropolis algorithm, chosen since it is simple to implement and to alter for the variety of different field contents and actions we are concerned with. In the cases where we have Grassmann-odd 
fields we include their contribution by calculating the fermion determinant directly. This would be expensive for a large number of modes (the cost scales as $n_{1}^{3}$ ) but is reasonable for the small number of modes in our simulations (we have at most $n_{1}=9$ ). In any case since the action is non-local the cost of evaluating it scales as $n_{0,1}^{3}$ even for the bosonic part.

Due to the phase-quenching, our errors increase as the imaginary part of the action increases, i.e. as we move to larger $x$. To some extent it is possible to compensate for this by increasing the number of configurations in our simulations, but the number of configurations required increases exponentially with $x$ so eventually this becomes impossible. The practical effect of this is that it gives an upper limit on the values of $x$ at which we can simulate; it will be very difficult to go much beyond this in future work.

There is no general method known to avoid the exponentially large cost associated with complex actions. In some specific cases the complex Langevin method (see [88] for a recent review), which does not have an exponential cost, can be used to bypass this 'sign problem'. The complex Langevin method is not a panacea, however; in some cases it converges to the wrong limit [89]. We attempted to bypass the sign problem by implementing the complex Langevin method for our system. We found results in agreement with the conventional Monte Carlo simulations at weak coupling, but disagreement at strong coupling, indicating that the complex Langevin method was converging to the wrong limit. Thus we did not pursue this method further.

As discussed in section 2.1.1, the action can be reformulated so that the quadratic terms are non-local while the cubic terms are local. The two formulations are equivalent and therefore should give identical results. Confirming that this is the case is a useful additional check of the correctness of our code. We have carried out this check for several sets of parameters and indeed found good agreement. The run time and statistical errors are similar for both formulations, so there is no particular benefit from using either case. We have chosen to use the formulation with the non-locality in the cubic term, and all our results below are for that case.

\subsection{Observables}

The observables we measure are the action $\langle S\rangle$, and the expectation values of the Grassmann even fields and their squares. The specific field content is dictated by the choice of scheme and level. For example, for scheme 4 , we measure $\left\langle T_{n}\right\rangle$ and $\left\langle T_{n}^{2}\right\rangle$ for all $l_{\max }$, and also $\left\langle A_{n}\right\rangle$ and $\left\langle A_{n}^{2}\right\rangle$ if $l_{\max }>1$. Here the subscript $n$ refers to the mode number, and we measure all modes present. We find that the $\left\langle A_{n}\right\rangle$ are always consistent with zero, in some cases with very small errors, of order $10^{-4}$. This is because $A$ only appears quadratically in the action - there are no terms linear or cubic in $A$. Hence we will not discuss $\left\langle A_{n}\right\rangle$ further.

One issue to be considered is whether or not to include the logarithm of the fermion determinant in the action when Grassmann-odd fields are present. (Here we refer to the action considered as an observable, not to the action used for the update algorithm, where of course the fermion determinant must be included.) The statistical weight used when Grassmann-odd fields are present is

$$
\operatorname{det} M e^{S_{B}}
$$


where $\operatorname{det} M$ is the fermion determinant and $S_{B}$ is the bosonic part of the action. This can be rewritten as

$$
e^{\ln \operatorname{det} M+S_{B}}
$$

The question is whether to take $S_{B}$ or $\left(\ln \operatorname{det} M+S_{B}\right)$ as the action. Neither of these is obviously more physical than the other, but in the weak-coupling limit, $S_{B}$ will simply be $-\frac{1}{2}$ per bosonic degree of freedom, whereas $\left(\ln \operatorname{det} M+S_{B}\right)$ will contain additional $L$-dependent terms coming from $\operatorname{det} M$. Because of this we have chosen to use $S_{B}$ as the action observable.

\subsection{Independence of the analytical continuation on the rotation angle}

As described above, we define the theory by an analytical continuation of the integration contour, which is implemented by a rigid rotation in the complex plane. So far we considered this rotation to be by an angle of $\frac{\pi}{6}$, which is exactly what is needed in order to make the cubic part of the action purely imaginary. We define $\gamma$ as the angle of rotation, i.e. $\gamma=0$ is the original theory and $\gamma=\frac{\pi}{6}$ is the angle that is needed for our analytical continuation.

Taking $\gamma=\frac{\pi}{6}$ has a large numerical cost, since then the action has a large imaginary part. Because of this, we use $\gamma=0$ when this is possible, i.e. when the cubic term is small. In some cases we found that it is possible to use intermediate values of $\gamma$ when the cubic term is not too large; this is worth it because even a small decrease in $\gamma$ from $\frac{\pi}{6}$ gives a large saving in computational cost.

When the instability is small it is possible to compare results for different values of $\gamma$ in the range $0 \leq \gamma \leq \frac{\pi}{6}$ in order to establish $\gamma$-independence. We have done this for several sets of parameters and obtained good agreement. For example, at $\alpha^{\prime}=1, V=-\sqrt{\frac{25}{6 \alpha^{\prime}}}$, $L=20, x_{\min }=-20$, and $l_{\max }=1.6$, we obtained

$$
\begin{array}{ll}
\left\langle T_{1}\right\rangle=-0.379(27)-0.140(18) i & \gamma=\frac{7 \pi}{48} \\
\left\langle T_{1}\right\rangle=-0.376(3)-0.146(4) i & \gamma=\frac{15 \pi}{96} \\
\left\langle T_{1}\right\rangle=-0.357(22)-0.164(19) i & \gamma=\frac{\pi}{6} .
\end{array}
$$

These results are clearly in good agreement. In this case, we found out that the metastability at $\gamma=\frac{7 \pi}{48}$ is manifested only around $1.7 \times 10^{7}$ updates (the result above was obtained from $10^{7}$ updates), while we did not observe the metastability at $\gamma=\frac{15 \pi}{96}$. Our results suggest that as long as the metastability does not manifest itself the results are almost $\gamma$-independent.

\section{$3.3 \quad \alpha^{\prime}$-independence}

$\alpha^{\prime}$, or equivalently $l_{s}(1.2)$, or $m_{s}(1.10)$, sets the scale for our simulations - for example the physical box size is $l_{s} L$. A useful check on the code is that it gives the same results for different $\alpha^{\prime}$ when all physical quantities (box size, $l_{\max }, g_{o}, \ldots$ ) are the same. ${ }^{12} \mathrm{We}$

\footnotetext{
${ }^{12} \mathrm{It}$ is important to remember that, as described in $1.1, g_{o}$ has dimension $\frac{5}{2}$.
} 
have carried out this check explicitly for the case $\alpha^{\prime}=1, L=20, x_{\min }=-20, l_{\max }=0.9$ and rescaled versions thereof, indeed obtaining identical results. Apart from this test, all the simulations have been carried out with $\alpha^{\prime}=1$; thus the lattice units are equivalent to string units.

\subsection{Estimate of statistical errors}

It is important to have reliable estimates of the statistical errors on our results, which we estimate using the jackknife method. This should provide accurate estimates, provided that there are no large auto-correlation times in the data, i.e. provided that configurations with large separation of lattice times are uncorrelated.

In a typical simulation we evaluate about $10^{9}$ updates, which we split into about 100 bins for analysis. The jackknife analysis is supposed to work well provided these bins are uncorrelated. Thus, the question is whether configurations $10^{7}$ updates apart are correlated. Since we have only about 20 degrees of freedom or less, it would be very surprising if this would have been the case.

As an additional check we have analysed how accurate the error estimates are. First, we generated high statistics data $\left(2 \times 10^{10}\right.$ configurations) for a particular set of parameters $\left(\alpha^{\prime}=1, V=-\sqrt{\frac{25}{6 \alpha^{\prime}}}, L=20, x_{\min }=-16, l_{\max }=0.9\right)$. This gave an accurate measurement of $\left\langle T_{1}\right\rangle$ with very small errors: $\left\langle T_{1}\right\rangle=-0.0749(8)-1.0317(8) i$. We then carried out ten independent low statistics runs $\left(2 \times 10^{6}\right.$ configurations each $)$ with the same parameters. Each of these gives an independent estimate of $\left\langle T_{1}\right\rangle$ with errors, and if the error estimate is correct these should all be consistent with the high-statistics result. For example, the first low-statistics run gave $\left\langle T_{1}\right\rangle=-0.123(92)-1.043(112) i$, which is indeed consistent.

Including both real and imaginary parts, this procedure gives 20 estimates with errors. 11 of these are within $1 \sigma, 19$ are within $2 \sigma$, and all are within $3 \sigma$ of the high-statistics result. This is completely consistent with the errors being estimated correctly, and indeed shows that any bias in the errors must be quite small.

\section{Results}

We focus our simulations on the issue of whether the theory becomes stable (or at least less unstable) in the limit where $l_{\max }$ and/or $L$ go to infinity. Recall, that we interpret stability as the vanishing of the imaginary parts of the various expectation values. We have no reason to expect that those will vanish already at the low level we work with here, but we would like to observe a tendency of decreasing the imaginary parts as compared to the real parts at least of some of the observables. In principle we would also like to take $x_{\max }$ to infinity, but as explained above this will not be possible and we will have to be content with taking it as far into the strong coupling region as we can. We expect that a sign of stability will be that the imaginary parts of observables go to zero, or at least decrease. Of course, it may be that this will work better for some observables than others. In particular, we might expect that it will work best for the lowest modes, which should be less affected by the missing higher level modes and fields. 
The results below are mainly for scheme 4, where we have the most detailed results, organised roughly into sections dealing with the effect of varying a single parameter (e.g. $L$, $V, \ldots)$ at a time. This is followed by briefer overviews of our results for the other schemes.

\subsection{A single mode}

A simple test of our code is to look at the case of a single mode of the field $T$, where we can compare to the analytical results of section 2.8 .

We can choose several sets of parameters that will give a single mode, for example $L=20, l_{\max }=0.05$, or $L=6, l_{\max }=0.9$, both with $\alpha^{\prime}=1$ and $V=-\sqrt{\frac{25}{6 \alpha^{\prime}}}$. These should give identical results, apart from an overall scale and shift in $x$ due to the different values of $a$ and $b$. We have found that this is indeed the case.

Another check is whether we obtain the correct limiting values. The case $\langle S\rangle=-\frac{1}{2}$ for $x_{\min } \rightarrow-\infty$ is easy to check: for example, for $L=6$ and $l_{\max }=0.9$ we obtain

$$
\begin{array}{ll}
S=-0.534(2)-0.0068(19) i ; & x_{\min }=-6 \\
S=-0.509(5)+0.0001(2) i ; & x_{\min }=-6.5,
\end{array}
$$

which is already very close.

The case $x_{\min } \rightarrow \infty$ is harder since the simulations become expensive in this limit. However, it is still doable, and for the same parameters we get

$$
\begin{array}{ll}
S=-0.334(2)-0.005(2) i ; & x_{\min }=-2 \\
S=-0.333(2)+0.001(2) i ; & x_{\min }=-1 .
\end{array}
$$

This is clearly going to the correct limit of $-\frac{1}{3}$.

Finally, we have looked at whether $T^{2}$ approaches the origin from the correct direction, i.e. at an angle of $\frac{2 \pi}{3}$ to the real axis. This is shown in figure 1 , again for the case $L=6$ and $l_{\max }=0.9$. We see that indeed the results appear to be going towards the right asymptotic line. All other observables approach zero as well, except for the action, which as described above goes to $-\frac{1}{3}$. (The reason the action is different is the factor of $b$ in eq. (2.89), which diverges in this limit.)

\subsection{Changing $l_{\max }$}

$l_{\max }$ is the highest level allowed for fields in the simulation. Since we only include fields up to $l_{0}=1$, it must be less than 2 . Increasing $l_{\max }$ means both allowing more fields (e.g. the $A$ field only appears for $l_{\max }>1$ ) and increasing the number of momentum modes of each field.

Since increasing $l_{\max }$ is like increasing the cutoff, we might hope that for high enough $l_{\max }$ the results will become independent of $l_{\max }$ (at least for some quantities). With this in mind we have done some scans in $l_{\max }$, keeping all other parameters fixed.

Generally we have concentrated on the observables $\langle S\rangle$ and $\left\langle T_{1}\right\rangle$ to simplify the presentation. However results for the other observables are similar. Throughout this section we fix $\alpha^{\prime}=1$ and $V=-\sqrt{\frac{25}{6 \alpha^{\prime}}}$. 


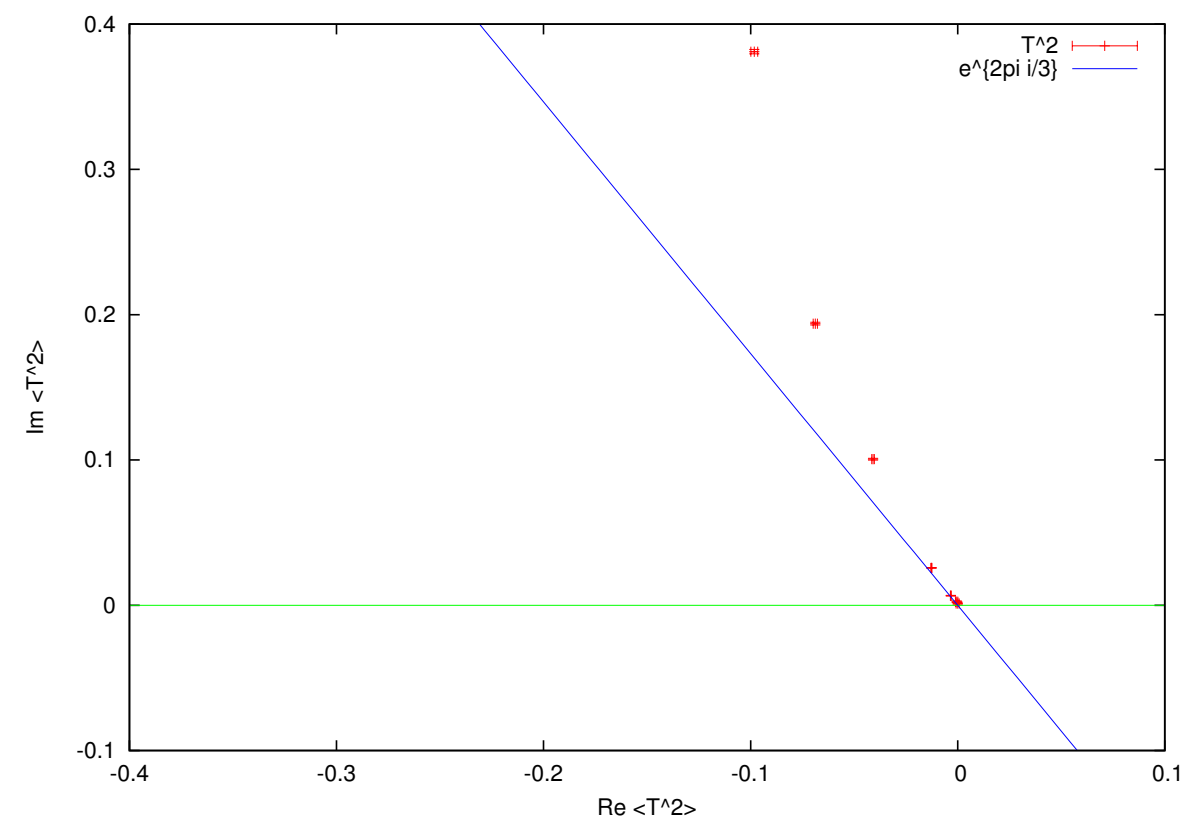

Figure 1. $\left\langle T^{2}\right\rangle$ in the complex plane for $L=6, l_{\max }=0.9$ for $x_{\min }$ in the range -3 to 2 (red), and the expected asymptotic line (blue).

\subsubsection{Extensive study at $L=20$}

We begin by describing our results for $L=20$, where we have the most extensive results. This value of $L$ is quite large, so there is a reasonable number of modes -9 for $T$ and 6 for $A$ up to $l=2$. We have done runs for every $l_{\max }$ between 0 and 2 which gives a different number of modes. We have also added runs where the level-1 fields $A, B$ and $C$ are not included in the simulation, to give some idea of the effect the level-1 fields have on the physics.

The strong coupling region begins around $x_{\min }=-20$, and we are able to get results with reasonably small errors up to $x_{\min }=-18$. We show results for the imaginary parts of the action and the $T_{1}$ mode in figures 2 and 3. Other observables show similar behaviour. The general trends we see are quite clear. First, increasing $l_{\max }$ increases $\Im\langle S\rangle$, and to a lesser extent $\Im\left\langle T_{1}\right\rangle$ as well. In particular, there is no evidence that the imaginary parts are going to zero as $l_{\max }$ is increased. There is not a great difference between the runs with and without the level-1 fields; in some cases they make the imaginary parts smaller, and in other cases larger. We also see a general trend for the imaginary parts to be larger at larger $x_{\min }$; this is not surprising as the destabilising cubic terms are becoming larger. Another point is that the results do not appear to become independent of $l_{\max }$ as it is increased, so we are not (yet?) seeing cutoff-independence.

From all of these, we also see that generally the results are smooth in $l_{\max }$. This means we can focus on a few key values of $l_{\max }$ to see the trends, which we often do from now on, especially in section 4.3. 


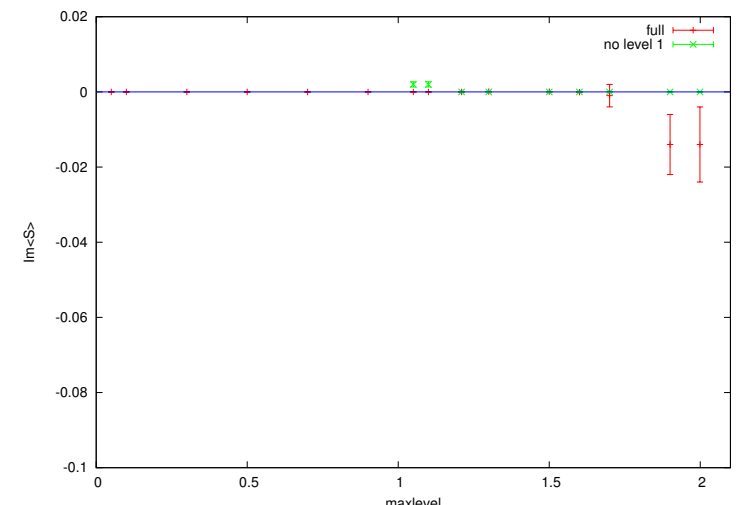

(a) $x_{\min }=-21$.

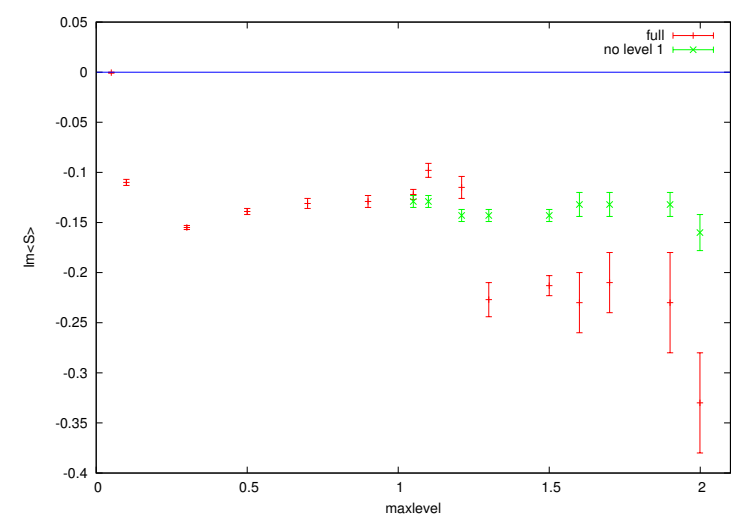

(c) $x_{\min }=-19$.

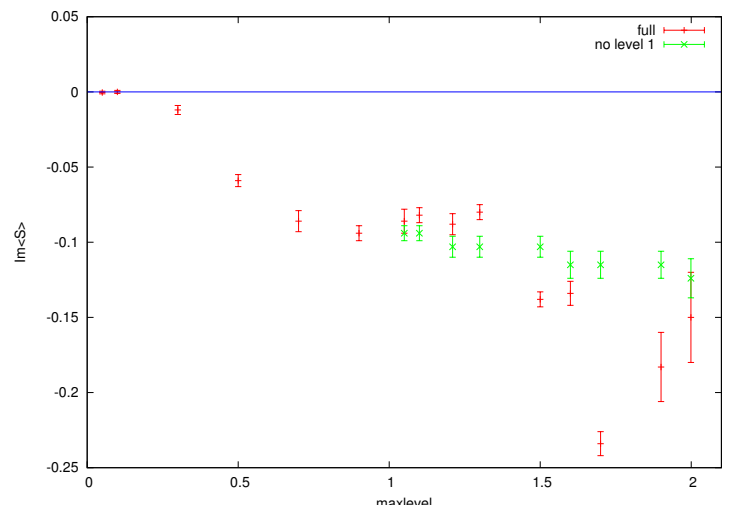

(b) $x_{\min }=-20$.

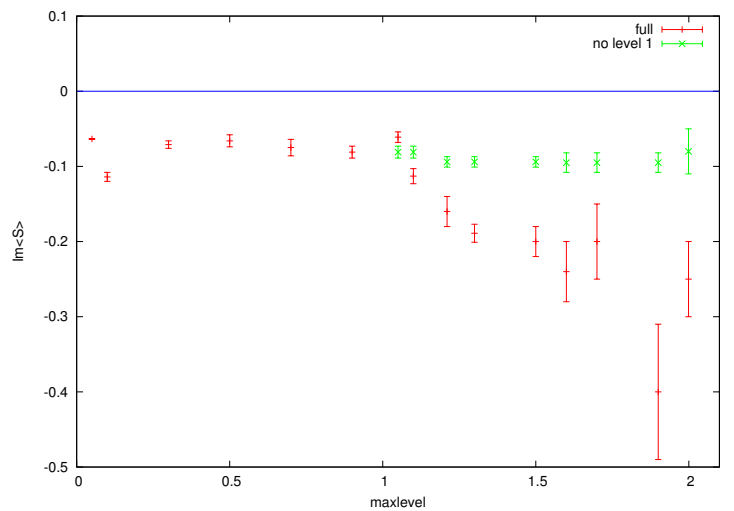

(d) $x_{\min }=-18$.

Figure 2. $\Im\langle S\rangle$ for $L=20$ for the full theory (red), and without level-1 fields (green), for $x_{\min }=-21,-20,-19,-18$.

\subsubsection{Results at $L=10$}

This is a much smaller interval, so there are fewer modes, specifically 4 for $T$ and 3 for $A$ up to $l=2$. There may potentially be a problem with having too few modes - e.g. one might imagine that one needs many modes to see "continuum" physics. On the other hand having fewer modes makes the simulations faster, so we can achieve smaller errors or go to stronger couplings. Note that because the numbers of level- 0 and level- 1 modes scale differently, there is no simple mapping between $L=10$ and $L=20$.

Again, we have carried out simulations for every $l_{\max }$ between 0 and 2 which gives a different number of modes. We have also added runs where the level-1 fields $A, B$ and $C$ are not included in the simulation, to give some idea of the effect the level-1 fields have on the physics.

The strong coupling region begins around $x_{\min }=-10$, and we are able to get results with reasonably small errors up to $x_{\min }=-8$. We show results for the imaginary parts of the action and the $T_{1}$ mode in figures 4 and 5 . Roughly speaking, we would expect results at a given $x_{\min }$ for $L=10$ to match those at $x_{\min }-10$ for $L=20$, since both will extend the same distance into the strong coupling region, so we have chosen the appropriate values of $x_{\min }$ to allow this comparison to be made with the results of section 4.2.1. In fact this turns 


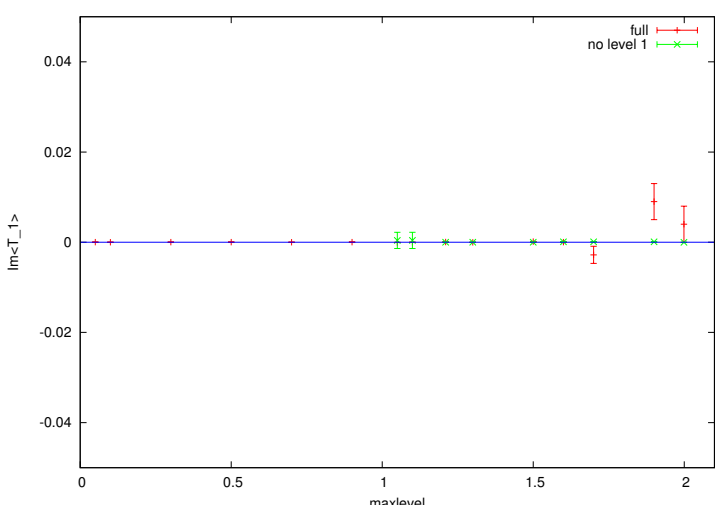

(a) $x_{\min }=-21$

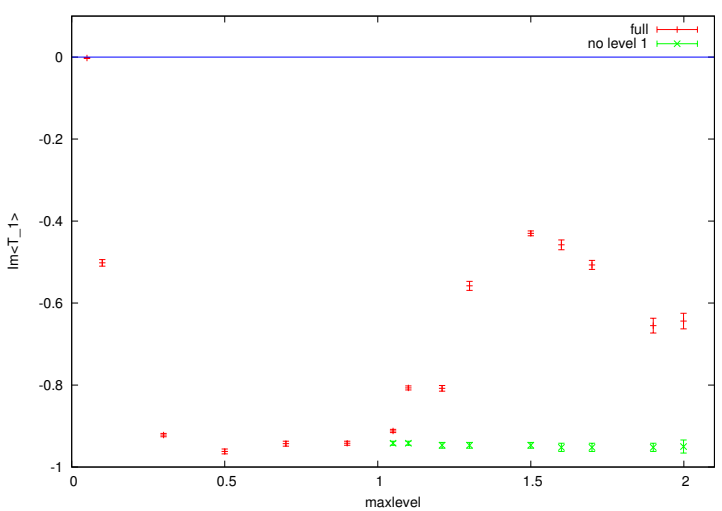

(c) $x_{\min }=-19$.

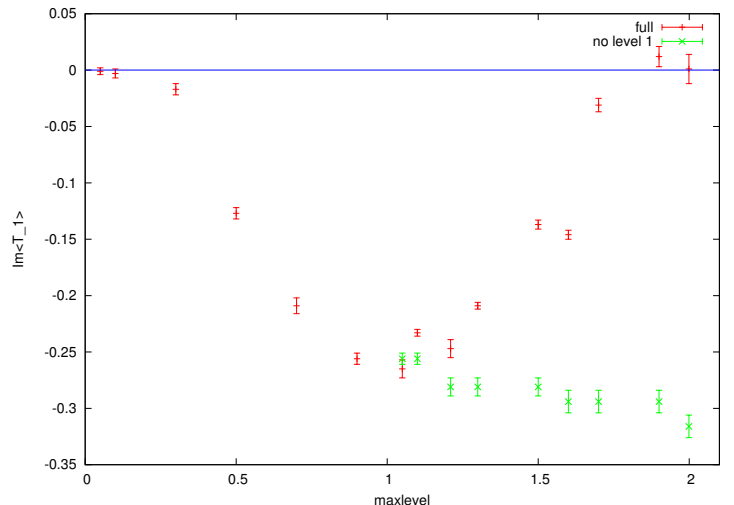

(b) $x_{\min }=-20$.

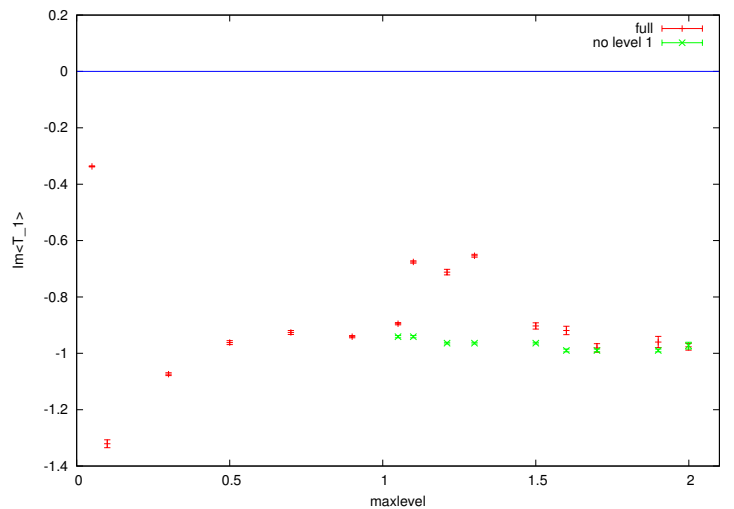

(d) $x_{\min }=-18$.

Figure 3. $\Im\left\langle T_{1}\right\rangle$ for $L=20$ for the full theory (red), and without level-1 fields (green), for $x_{\min }=-21,-20,-19,-18$.

out to be not exactly true, and we see no instability at all at $x_{\min }=-11$ (the imaginary parts are at most of order $10^{-5}$ ), so we do not show any plots for this case.

We see that the results look rather similar to those for $L=20$. One potentially interesting area in these plots is just above $l_{\max }=1$, since the density of level-1 modes is much higher here for $L=20$ than for $L=10$. However, comparing the plots, nothing interesting seems to happen in this range. Overall, it appears there is not much difference between $L=10$ and $L=20$, which at least suggests that the number of modes we are looking at is not too small.

\subsubsection{Including a large number of modes at $L=30$}

This is a large interval, with the number of modes and their density also becoming quite large - specifically we have $n_{0}=13$ and $n_{1}=9$ for $l_{\max }=2$. If there is any effect that requires many modes, it would be surprising if it did not yet appear here. However, the large number of modes also makes $L=30$ very expensive. ${ }^{13}$ The largest $x_{\min }$ we have been able to reach is $x_{\min }=-30$, corresponding to $x_{\max }=0$ and so only at the beginning of strong coupling.

\footnotetext{
${ }^{13}$ The computational cost goes very roughly as $n_{0}^{3}$.
} 


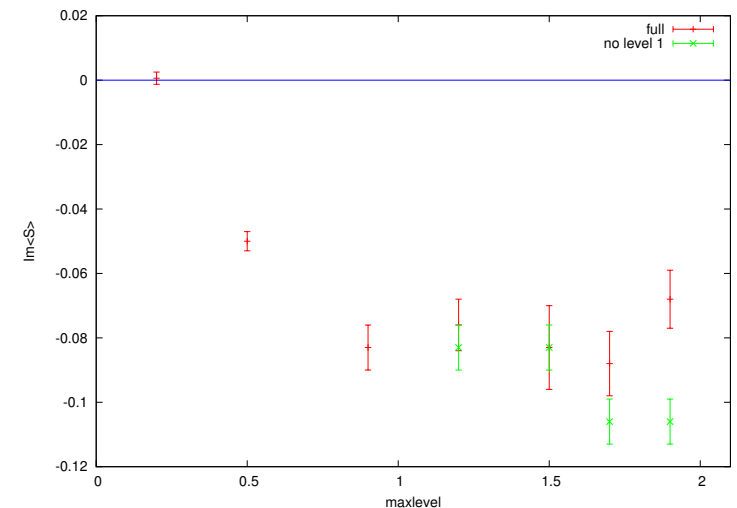

(a) $x_{\min }=-10$.

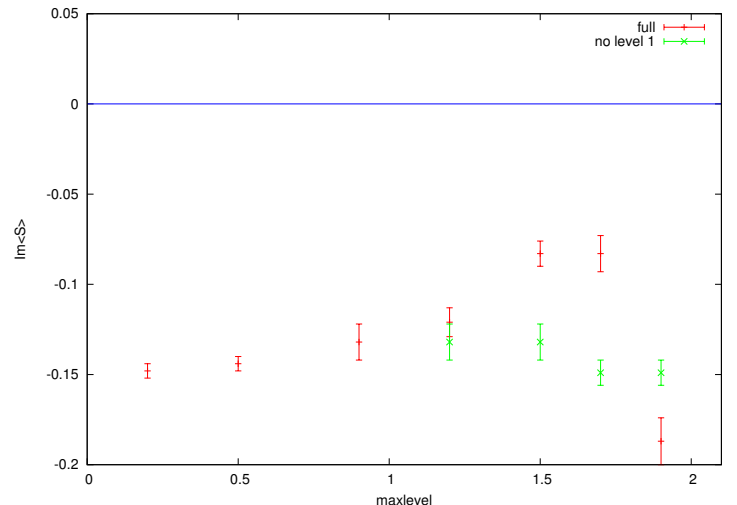

(b) $x_{\min }=-9$.

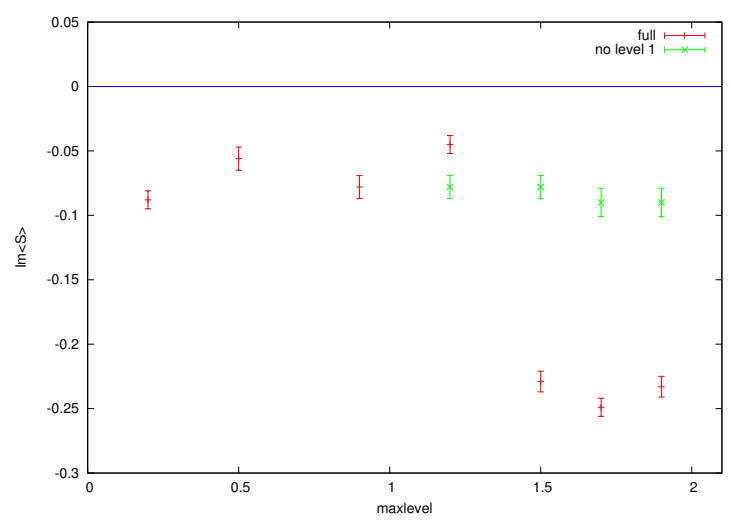

(c) $x_{\min }=-8$.

Figure 4. $\Im\langle S\rangle$ for $L=10$ for the full theory (red), and without level-1 fields (green), for $x_{\min }=-10,-9,-8$.

Again, we have results for every $l_{\max }$ between 0 and 2 which gives a different number of modes. We show results for the imaginary parts of the action and the $T_{1}$ mode in figures 6 and 7 . The overall results look rather similar, though in detail they are different; for example, $\Im\left\langle T_{1}\right\rangle$ is now positive for some values of $l_{\max }$, which was not the case for smaller $L$.

Unlike for the case of $L=20, x_{\min }=-21$, and $L=10, x_{\min }=-11$ we see that at $x_{\min }=-31$ there are rather large imaginary parts for the larger values of $l_{\max }$. Therefore it looks like increasing $L$ at constant $x_{\max }$ is destabilising. This seems a bit counterintuitive when we think about it another way: going from $L=20, x_{\min }=-21$ to $L=30$, $x_{\min }=-31$ is a change in $x_{\min }$ while keeping $x_{\max }$ fixed, so we are only adding a region of extremely weak coupling - how can this make things less stable? Probably the answer is connected to the fact that increasing $L$ means we have more modes, and thus a shift in one boundary affects the physics throughout the interval. Moreover, the non-locality of the action might also source some influence of one boundary on the rest of the interval. These effects lead to a worsening of the numerical sign problems and therefore to larger errors. 


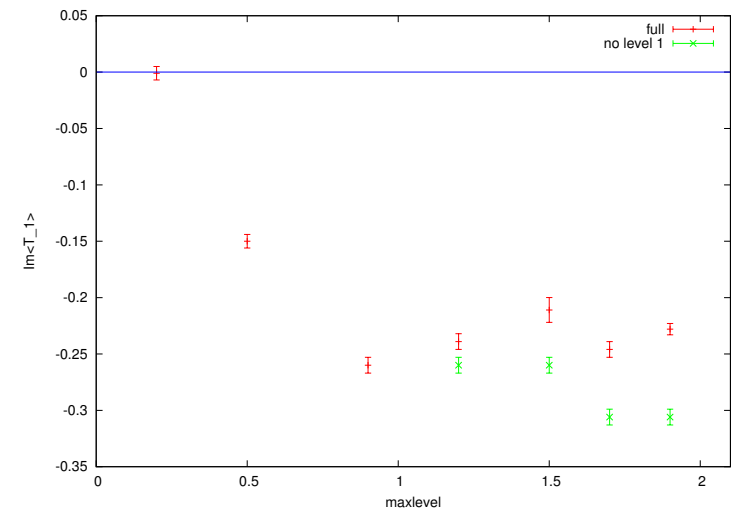

(a) $x_{\min }=-10$.

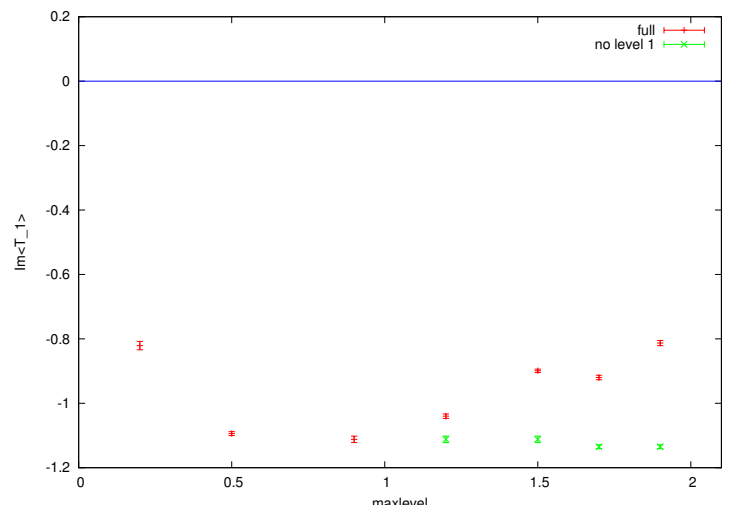

(b) $x_{\min }=-9$.

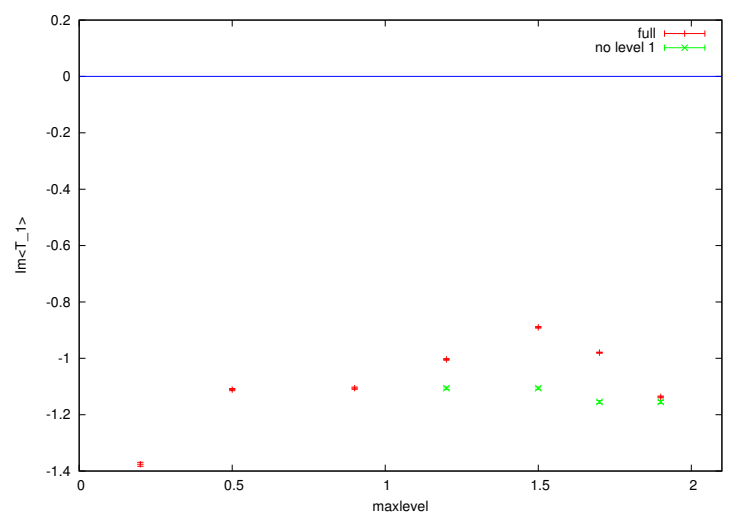

(c) $x_{\min }=-8$.

Figure 5. $\Im\left\langle T_{1}\right\rangle$ for $L=10$ for the full theory (red), and without level-1 fields (green), for $x_{\min }=-10,-9,-8$.

\subsection{Changing $x_{\text {min }}$}

$x_{\min }$ is a very important parameter as it controls the strength of the cubic terms and hence the instabilities. We expect that for small $x_{\min }$ there will be no instabilities and so the imaginary parts of observables will be zero. They will then increase, and finally, if they behave as in the case of a single mode (see section 4.1) go to zero (except for the action which should go to a finite value).

Since we have established above that the observables have a rather smooth dependence on $l_{\max }$, we have mostly concentrated on a few values of $l_{\max }$. We do not present results for $L=30$ in this section since, as discussed above, we have been unable to reach very strong couplings in this case.

\subsubsection{Extensive study at $L=20$}

We begin again with our results for $L=20$, where we have the most data. For most of this scan in $x_{\min }$, we only use the values $l_{\max }=\{0.05,0.5,0.9,1.5,1.999\}$. We also include $l_{\max }=\{1.5,1.999\}$ with level-1 fields removed. We have tried to reach the highest values 


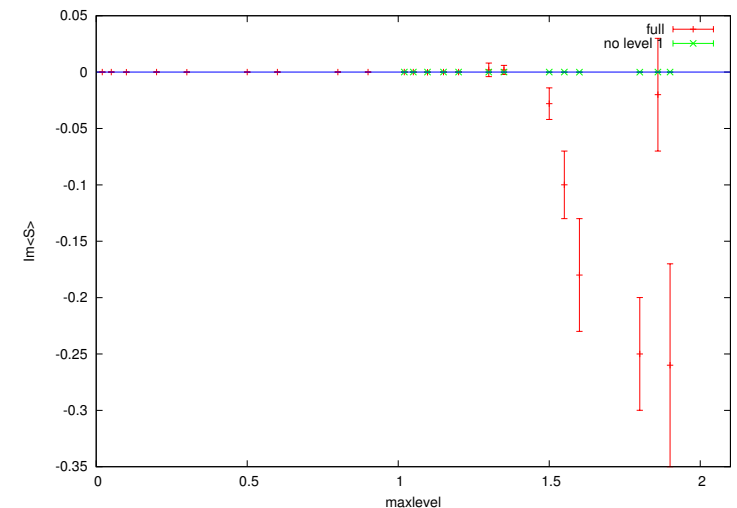

(a) $x_{\min }=-31$.

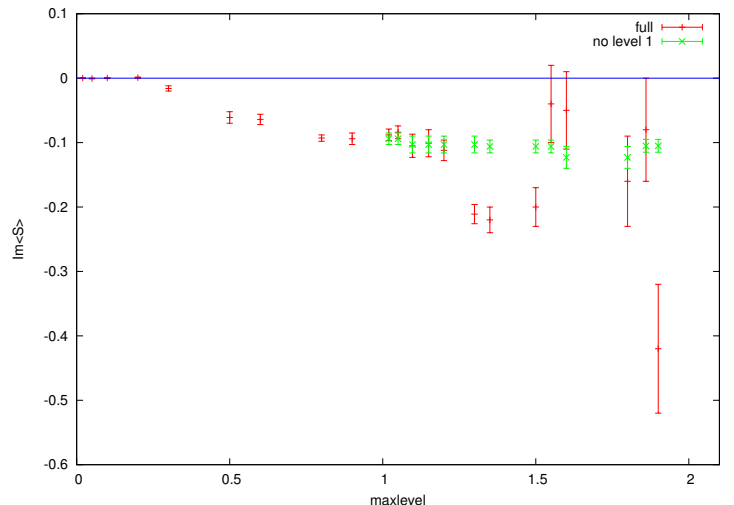

(b) $x_{\min }=-30$.

Figure 6. $\Im\langle S\rangle$ for $L=30$ for the full theory (red), and without level-1 fields (green), for $x_{\min }=-31$ and -30 .

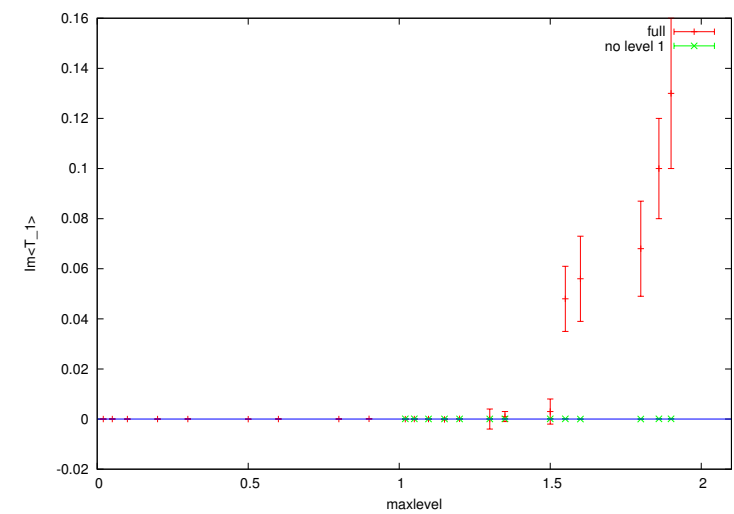

(a) $x_{\min }=-31$.

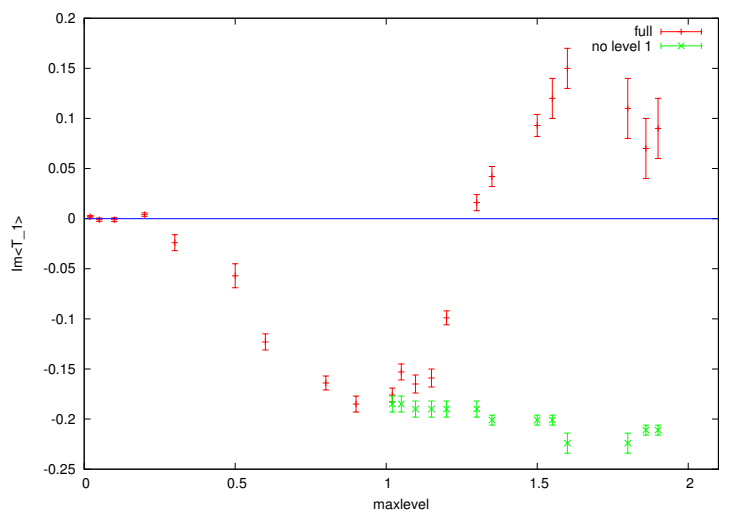

(b) $x_{\min }=-30$.

Figure 7. $\Im\left\langle T_{1}\right\rangle$ for $L=30$ for the full theory (red), and without level-1 fields (green), for $x_{\min }=-31$ and -30 .

of $x_{\text {min }}$ possible, in order to try to reach the extreme strong-coupling region where all the observables go to zero. In practice this has only been possible for the lower values of $l_{\max }$.

First we show a plot of $\langle S\rangle$, in figure 8, showing both the behaviour of the imaginary part and the trajectory in the complex plane. We see that there is a similar trend for all $l_{\max }$. This even includes the single-mode $l_{\max }=0.05$, except for a shift in $x_{\min }$. Presumably the fact that there is a single mode means that there is less instability. Actually, this is the opposite of what we would expect from the hope that "the instabilities become of zero measure" as the number of degrees of freedom increases.

In the complex plane, it appears that the action executes a qualitatively similar 'loop' for all $l_{\max }$. In all cases the imaginary part only seems to become zero again when the trajectory reaches its final point, although for the higher $l_{\max }$ the data does not go far enough to be certain of this. Note that the large offsets between the loops for the different $l_{\max }$ are simply due to the $-1 / 2$ of action per mode that we get without the cubic terms. 


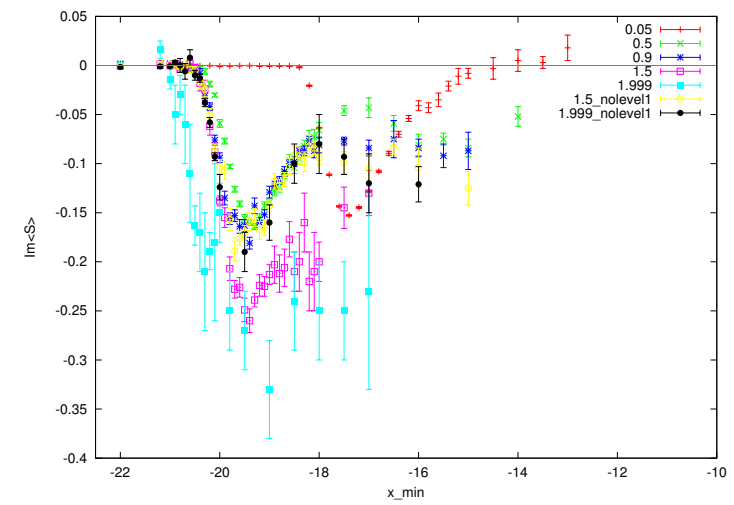

(a) $\Im\langle S\rangle$.

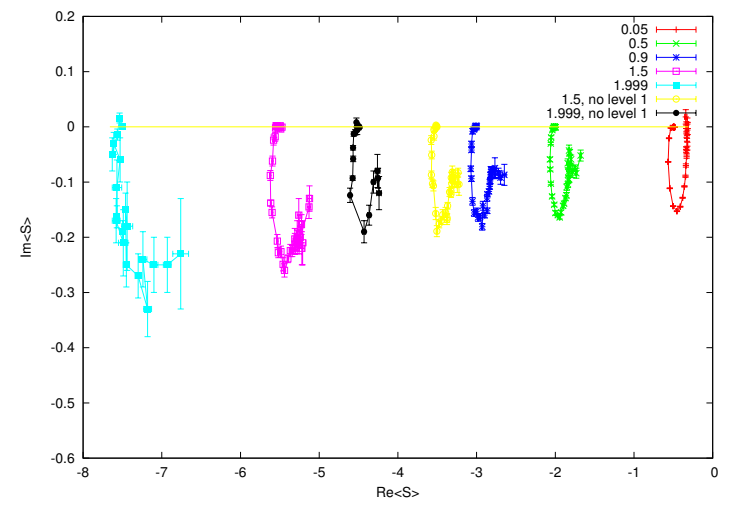

(b) Trajectory of $\langle S\rangle$ in the complex plane. In all cases increasing $x_{\text {min }}$ corresponds to anticlockwise movement along the trajectory.

Figure 8. $\langle S\rangle$ for $L=20$ as a function of $x_{\min }$ for various $l_{\max }$.

Apart from this all the $l_{\max }$ are rather similar — things appear to get neither better nor worse as more modes are added.

In some cases there appear to be cusps in the trajectories in figure 8, although this is not quite clear. If they are present they are probably related to the loops and cusps seen at small $L$ (see section 4.4 below.)

The imaginary part of $\left\langle T_{1}\right\rangle$ has a similar behaviour, plotted in figure 9 . In this case the return to zero is slower, but this is partially compensated for by the fact that the errors are smaller so we are able to go to higher $x_{\min }$. Again there is not much difference between the different values of $l_{\max }$, except for $l_{\max }=0.05$, where again the instability begins at higher $x_{\min }$. There are clear oscillations in the data; these correspond to cusps in the complex plane like those seen for the action. (These can be seen more clearly at smaller $L$, e.g. in figure 13.)

\subsubsection{Results at $L=10$}

This is quite a small interval, but has the advantage that it is possible to get to stronger coupling than in the $L=20$ case. We use the values $l_{\max }=\{0.2,0.9,1.9\}$, and also $l_{\max }=1.9$ with level-1 fields removed.

We begin with our results for $\langle S\rangle$, which are plotted in figure 10. The main features are the same as for $L=20$, but now we can follow them to stronger coupling, giving us more confidence in what happens there. It is clearer that $\langle S\rangle$ is returning to the real axis for all $l_{\text {max }}$. The cusps in the complex plane are still there, and the single-mode case (in this case $\left.l_{\max }=0.2\right)$ again becomes unstable at larger $x_{\min }$ than the others, although the difference seems smaller this time.

For $\Im\left\langle T_{1}\right\rangle$, plotted in figure 11, we can follow the behaviour almost back to $\Im\left\langle T_{1}\right\rangle=0$ for some $l_{\max }$. In general, the behaviour is similar to that for $L=20$. The oscillations are still present, for example for $l_{\max }=0.9$, though they are harder to see as we have not sampled so densely in $x_{\min }$. 


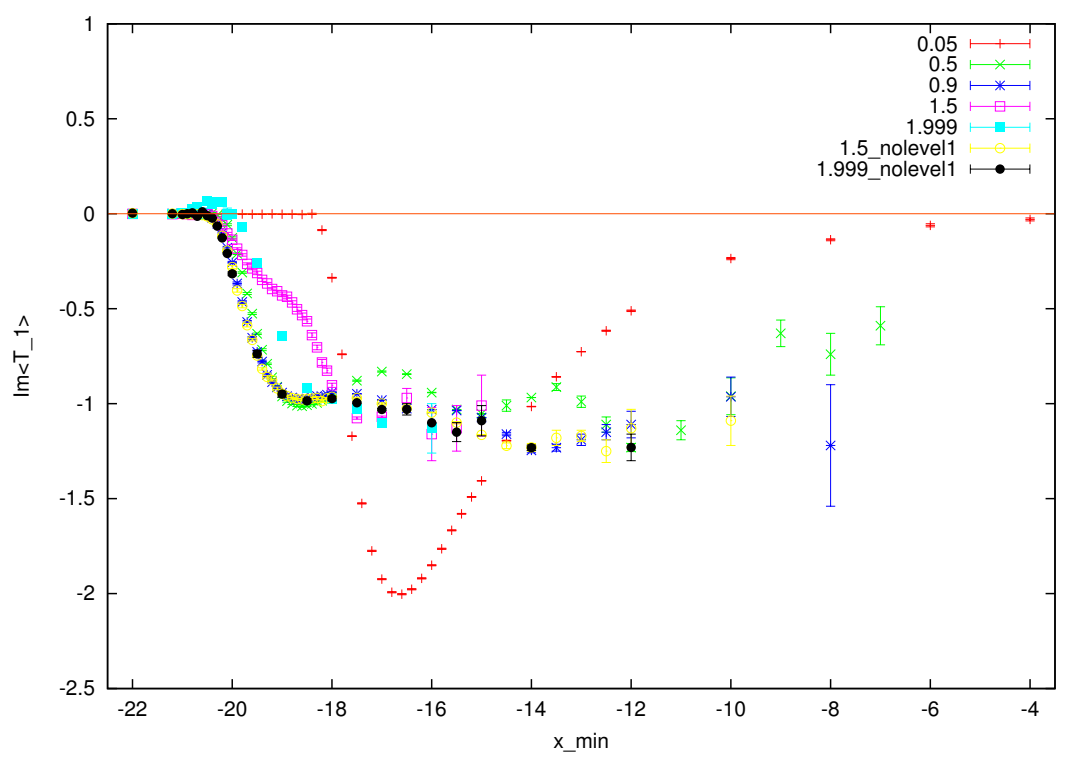

Figure 9. $\Im\left\langle T_{1}\right\rangle$ for $L=20$ as a function of $x_{\min }$ for various $l_{\max }$.

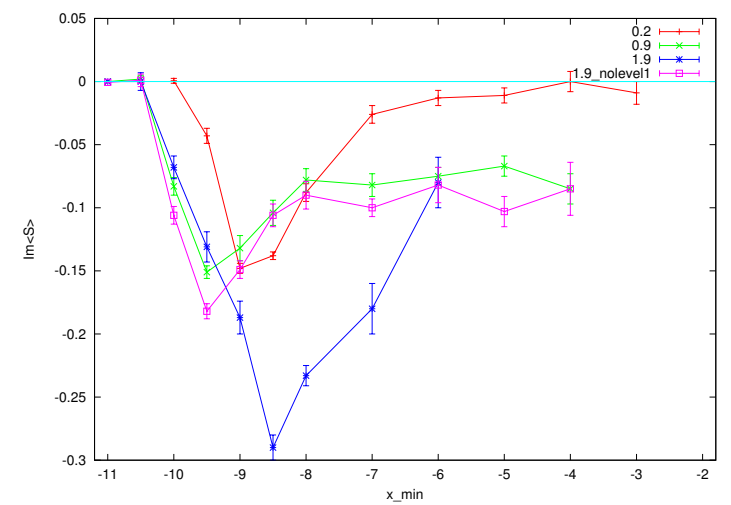

(a) $\Im\langle S\rangle$.

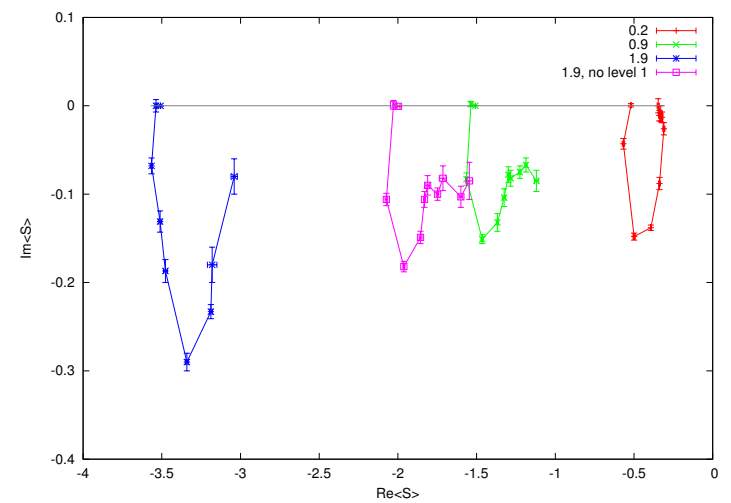

(b) Trajectory of $\langle S\rangle$ in the complex plane. In all cases increasing $x_{\min }$ corresponds to anticlockwise movement along the trajectory.

Figure 10. $\langle S\rangle$ for $L=10$ as a function of $x_{\min }$ for various $l_{\max }$.

\subsection{Small interval length}

Some of the results in the sections above are rather complicated, due to the presence of many modes. This is probably necessary to reach the large-volume and continuum limits. However, it may also be useful to look at a small number of modes to try to interpolate between the well-understood single mode case (see section 4.1) and the more complicated cases with many modes. Another advantage is that by keeping the number of modes small we can go to stronger couplings.

In this section we keep the number of modes small by taking the interval length $L$ small, specifically 6 or less. Note that the smallest value of $L$ we can take while keeping at least one mode below level 2 is $L=\pi / \sqrt{2} \approx 2.22$, and that for $L<\pi$ we have only a single mode. 


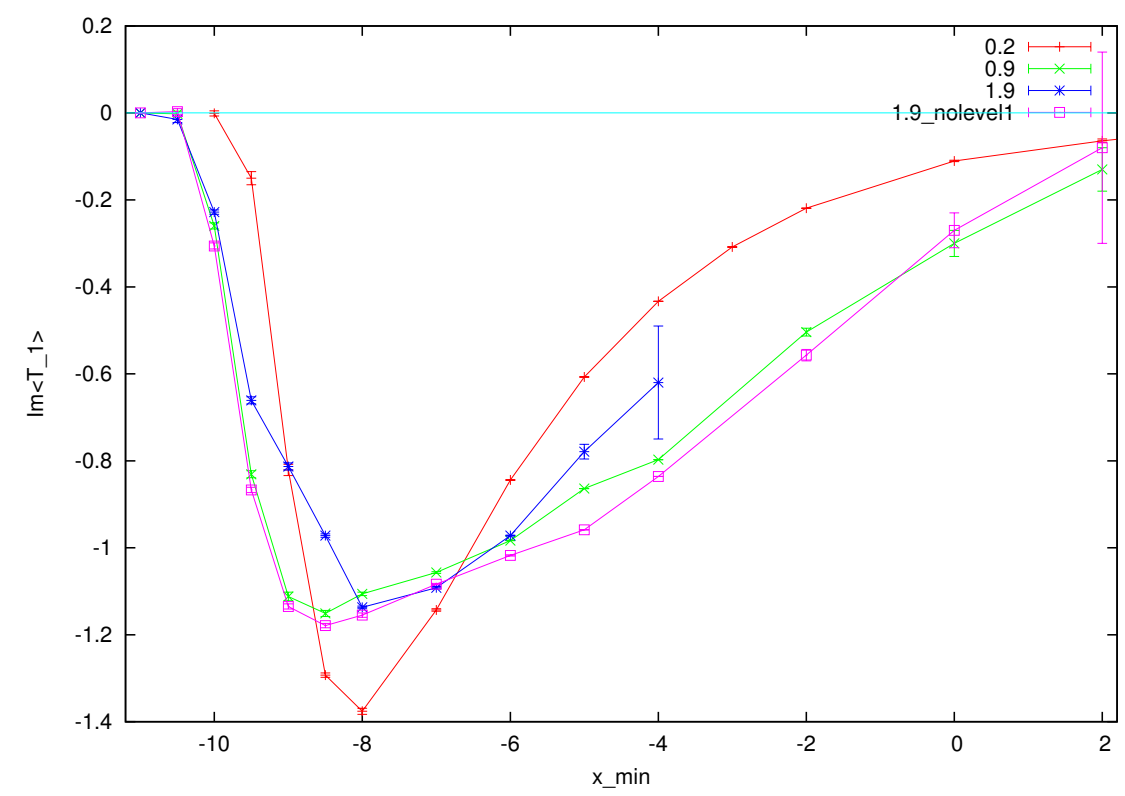

Figure 11. $\Im\left\langle T_{1}\right\rangle$ for $L=10$ as a function of $x_{\min }$ for various $l_{\max }$.

Another reason to look at small $L$ is that it may be possible that the continuum and large-volume limits are tied together, such that we need to take $L$ and $l_{\max }$ to infinity while keeping their ratio $l_{\max } / L$ fixed. Such a requirement seems natural, for example, from the perspective of T-duality. Since we are restricted to $l_{\max }<2$ we may need to take $L$ small too, to keep this ratio at least moderately large.

\subsection{1 $L=6$}

For $L=6$ the maximum number of modes for $l_{\max }$ below 2 is two for the $T$ field and one for the $A$ field. Since the number of modes is small, we can look at all of them without the plots becoming excessively complicated. We first plot $\langle S\rangle$ and $\left\langle T_{1}\right\rangle$ in figures 12 and 13 respectively.

The case $l_{\max }=0.9$ is the single-mode case, and we see this behaves as usual (see section 4.1). However the behaviour for $l_{\max }=1.2$ and in particular $l_{\max }=1.9$ is very different from the behaviour seen at larger $L$. There are very large oscillations in $\Im\langle S\rangle$, and an inflection point in $\Im\left\langle T_{1}\right\rangle$. From the right-hand panels of these figures we see that the oscillation in $\Im\langle S\rangle$ is actually a loop in the complex plane, and the inflection point in $\Im\left\langle T_{1}\right\rangle$ is actually a cusp.

The remaining observables ${ }^{14}$ are $T_{2}, T_{1}^{2}, T_{2}^{2}$, and $A_{1}^{2}$ (the superscripts are powers). These give a somewhat mixed picture: the trajectories in the complex plane look smooth for $T_{2}$ and $A_{1}^{2}$, but have cusps for $T_{1}^{2}$ and $T_{2}^{2}$. In general, they become cuspier as $l_{\text {max }}$ increases.

We do not understand what the cause of these loops and cusps is. One odd feature is that the cusps do not appear in all variables at the same $x_{\min }$. For example, for $l_{\max }=1.9$,

\footnotetext{
${ }^{14}$ There is also $A_{1}$, but this is always consistent with zero. We could have studied also expectation values of higher powers of the fields, but didn't do that for simplicity.
} 


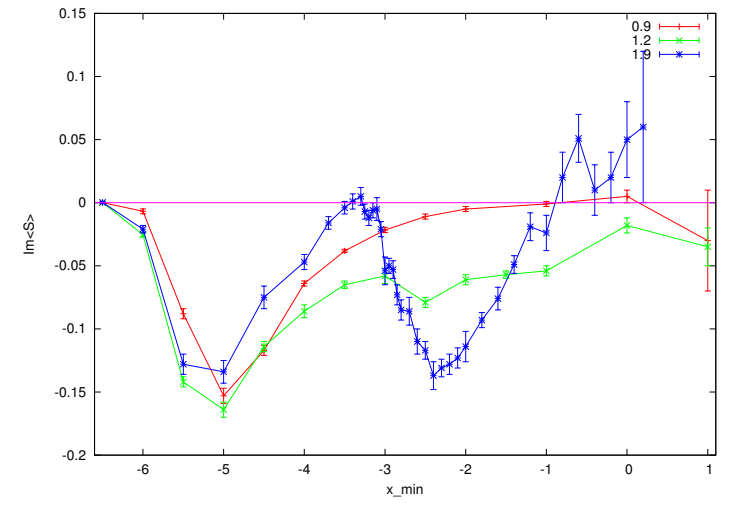

(a) $\Im\langle S\rangle$.

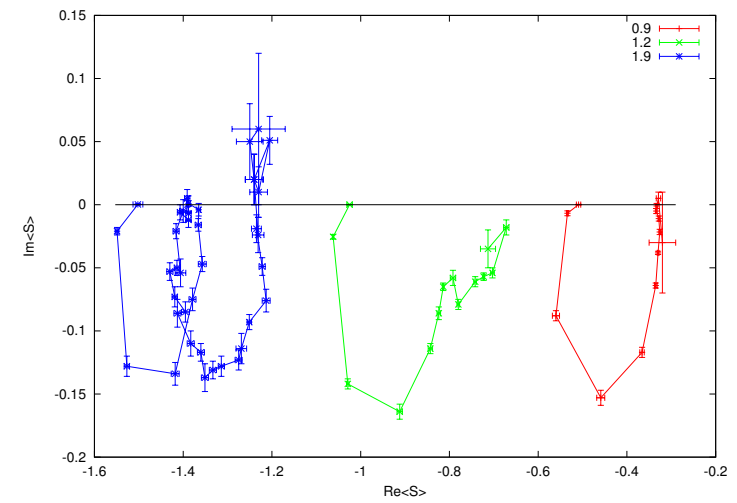

(b) Trajectory of $\langle S\rangle$ in the complex plane. In all cases increasing $x_{\text {min }}$ corresponds to anticlockwise movement along the trajectory.

Figure 12. $\langle S\rangle$ for $L=6$ as a function of $x_{\min }$ for various $l_{\max }$.

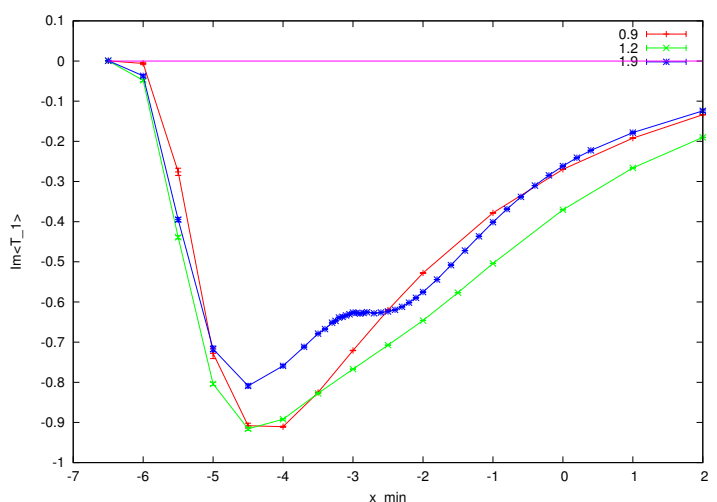

(a) $\Im\left\langle T_{1}\right\rangle$.

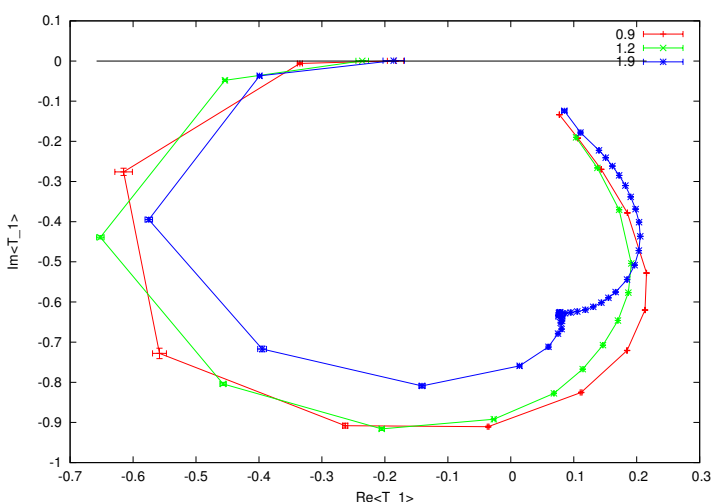

(b) Trajectory of $\left\langle T_{1}\right\rangle$ in the complex plane. In all cases increasing $x_{\min }$ corresponds to anticlockwise movement along the trajectory.

Figure 13. $\left\langle T_{1}\right\rangle$ for $L=6$ as a function of $x_{\min }$ for various $l_{\max }$.

the cusp in $T_{1}$ is around $x_{\min }=-2.9$, and $T_{2}^{2}$ is smooth in this region, and the cusp in $T_{2}^{2}$ is around $x_{\min }=-4.0$, where $T_{1}$ is smooth. This possibly seems to indicate that they do not have a single cause.

Another issue is whether these features are present at larger $L$. It is in fact quite likely that they are present, but that they are more difficult to see. This is for two reasons: firstly the large errors will obscure small features in the complex plane, and secondly it is not possible to go to as strong couplings, where the features seem to show up. In fact there are some indications for features like this at larger $L$ in some of the plots, e.g. in figure 10, and the oscillations in figure 9 .

For large $x_{\min }$ nearly all the observables approach the origin. The exception is the action, where we already saw for the case of a single mode that the action went to a finite value despite the fact that $T^{2}$ and $T^{3}$ go to zero. 
However, it is still interesting to ask how the observables approach the origin. In particular, do they approach along the real axis? If so this would be a good sign, since it would mean that as the origin is approached the ratio of imaginary part and the real part tends to zero. This is not necessary, but could be an indication that we really approach a good limit, since for expectation values that approach a non-zero real limit this ratio tends to zero regardless of the angle of approach. However, this does not seem to happen for any of the quantities we measure; they all approach the origin from a complex direction. There is also no trend of this getting better as $l_{\max }$ increases.

\subsection{2 $L=4$}

In this case the maximum number of modes is two; in comparison to $L=6$ we have lost the $T_{2}$ "tachyon" mode. Any significant differences between $L=4$ and $L=6$ could thus be due to having more than one mode of the $T$ field.

We show $\langle S\rangle$ and $\left\langle T_{1}\right\rangle$, in figures 14 and 15 respectively. We see a change in behaviour: $\Im\langle S\rangle$ becomes positive for $l_{\max }=1.9$ and large $x_{\min }$, corresponding to a large loop in the complex plane. Also $\left\langle T_{1}\right\rangle$ has a rather sharp curve, although not a cusp, for $l_{\max }=1.9$. Note that $\langle S\rangle$ has only a single loop, whereas for $L=6$ it had two; it is tempting to speculate that this is connected to the number of modes of the $T$ field in each case.

The remaining observables, that is $T_{1}^{2}$ and $A_{1}^{2}$, behave similarly to $L=6$. Taking all the observables together, the differences from $L=6$ are not very great. In particular, the sizes of the imaginary parts are similar, and there is no change in the tendency to approach the origin from a complex direction (as opposed to along the real axis). The biggest difference is probably that the cusps, loops etc. are more pronounced and more complicated for $L=6$.

\subsection{3 $L=3.15$}

Finally, we report results for $L=3.15$. This is almost the smallest interval we can have while keeping more than one mode - the second mode would go above level 2 at $L=\pi$. The modes present are in fact the same as at $L=4$; it is just their masses and the cubic terms that are different. For example, evaluating the masses explicitly, we find for $L=4$

$$
\begin{aligned}
& m_{T_{1}}=0.812 \ldots \\
& m_{A_{1}}=1.288 \ldots,
\end{aligned}
$$

and for $L=3.15$

$$
\begin{aligned}
& m_{T_{1}}=1.018 \ldots \\
& m_{A_{1}}=1.427 \ldots .
\end{aligned}
$$

So as we decrease $L$ the masses increase, while coming closer together.

We find that in general the results are rather similar to those for $L=4$. As an example we show the action in the complex plane in figure 16, where clearly the differences are rather small. We find similar results for the other observables. This suggests that the changes in the number of modes, rather than the changes in the parameters in the action, are more important. Also, this is the largest $l_{\max } / L$ we are able to reach, and nothing very helpful seems to happen. 


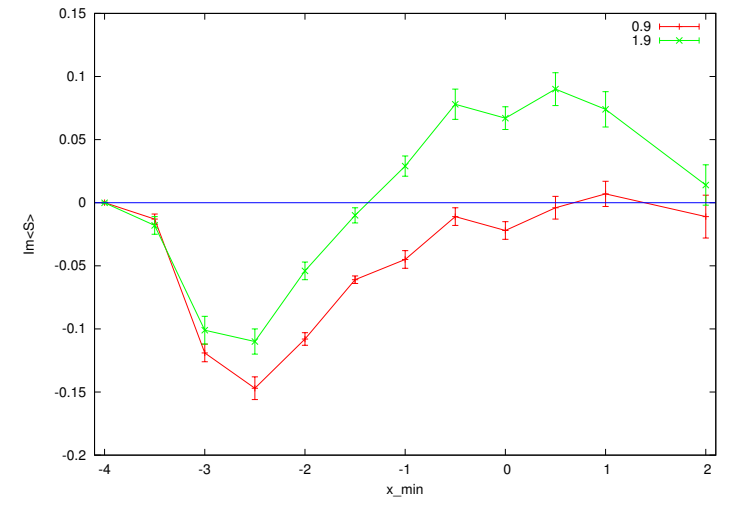

(a) $\Im\langle S\rangle$.

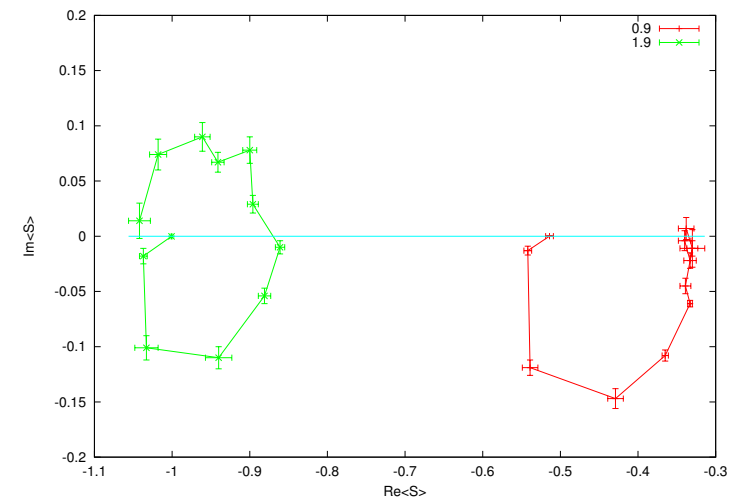

(b) Trajectory of $\langle S\rangle$ in the complex plane. In all cases increasing $x_{\text {min }}$ corresponds to anticlockwise movement along the trajectory.

Figure 14. $\langle S\rangle$ for $L=4$ as a function of $x_{\min }$ for various $l_{\max }$.

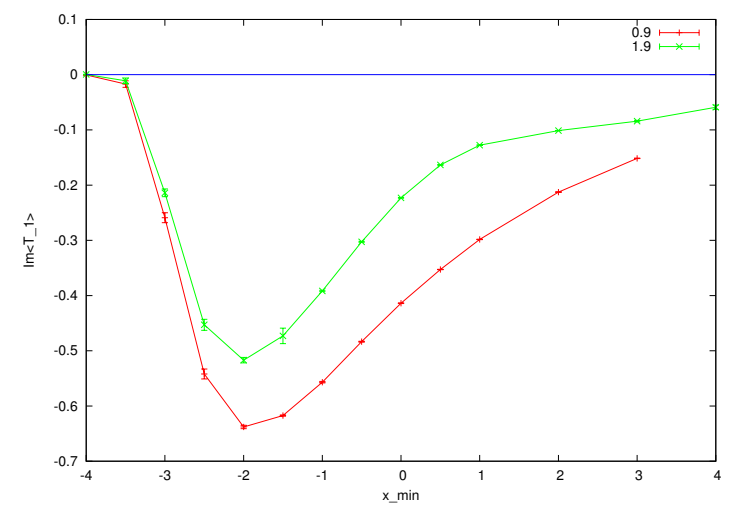

(a) $\Im\left\langle T_{1}\right\rangle$.

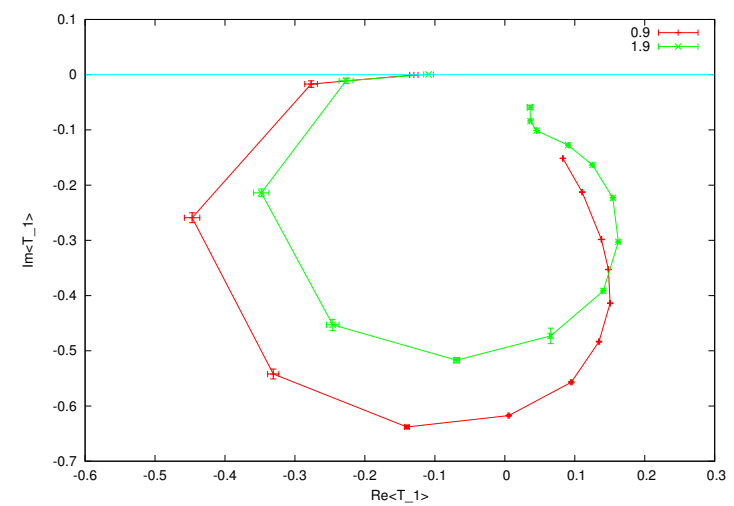

(b) Trajectory of $\left\langle T_{1}\right\rangle$ in the complex plane. In all cases increasing $x_{\min }$ corresponds to anticlockwise movement along the trajectory.

Figure 15. $\left\langle T_{1}\right\rangle$ for $L=4$ as a function of $x_{\min }$ for various $l_{\max }$.

\subsection{Comparing different interval lengths}

In this section we do not present any results not already mentioned above. Instead we show some plots comparing results at different $L$, mostly showing that there are no strong trends in $L$. In particular, there is not much sign of instabilities reducing as $L$ increases. This would be seen by either the imaginary parts of observables getting smaller, or by the approach to the origin at large $x_{\max }$ happening at a smaller angle to the real axis (or both).

When comparing different $L$, it is not always clear what are the equivalent things to compare. For example, if $L$ is doubled, should the mode $T_{1}$ be now compared to $T_{2}$, which has the same wavelength? Or should it still be compared to $T_{1}$, which is the lowest mode? We will mention issues of this sort as they become relevant below.

First we compare $\langle S\rangle$ at two different values of $l_{\max }$ for $L=6,10$ and 20, in figure 17 . We see that in both cases there is a very large shift of $\langle S\rangle$ for the different $L$. However, 


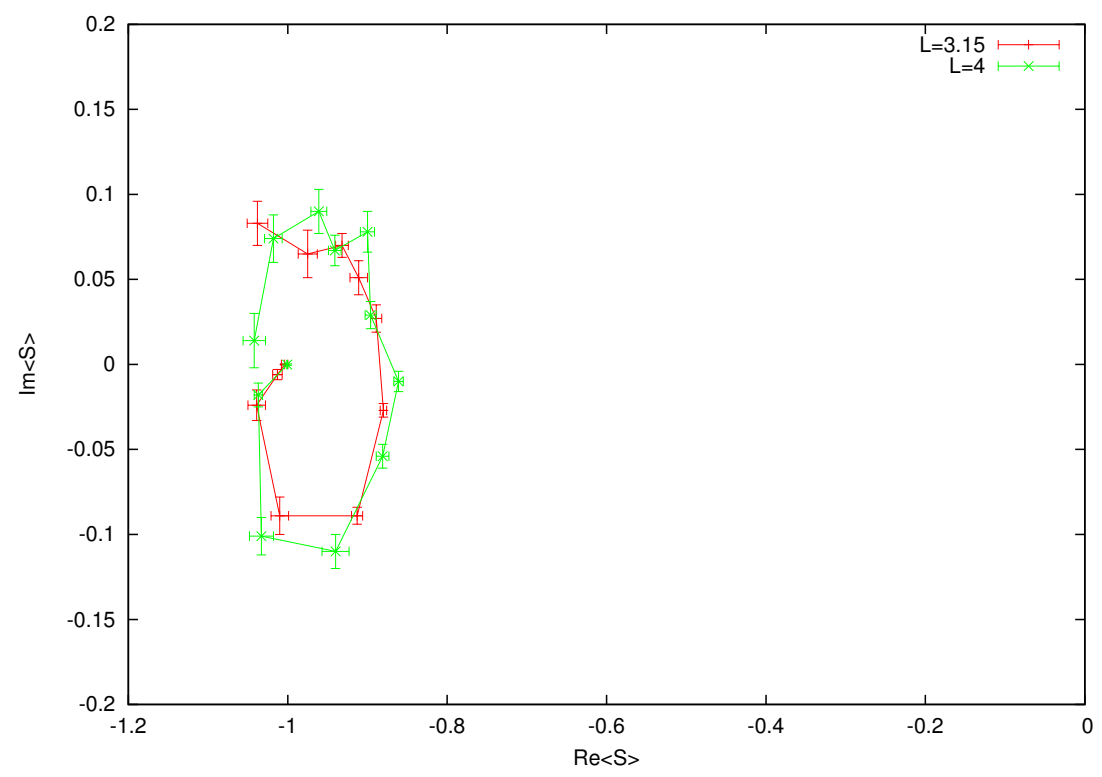

Figure 16. Trajectory of $\langle S\rangle$ in the complex plane for $L=4$ and $L=3.15$, both for $l_{\max }=1.999$. Increasing $x_{\min }$ corresponds to anticlockwise movement along the trajectory.

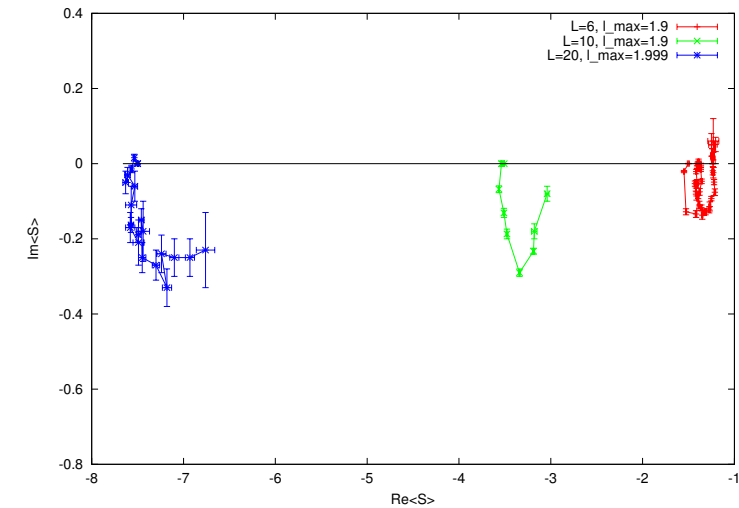

(a) $l_{\max }=1.999$.

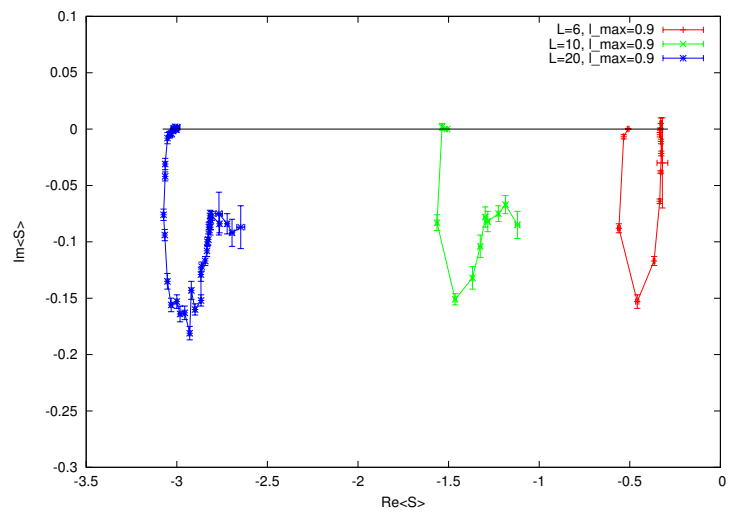

(b) $l_{\max }=0.9$.

Figure 17. Trajectory of $\langle S\rangle$ in the complex plane for $L=6$ (red), $L=10$ (green), and $L=20$ (blue). Increasing $x_{\min }$ corresponds to anticlockwise movement along the trajectory.

most of this is just due to the fact that the number of modes is increasing and the free action is $-1 / 2$ per mode. Apart from this we see a small increase in the size of $\Im\langle S\rangle$. There is also perhaps a tendency for the trajectories to become more complicated for higher $L$.

We now turn to comparisons of the field $T$. First we consider the lowest mode $T_{1}$, for which we show a plot in figure 18. The cases $L=6, l_{\max }=0.9$ and $L=10, l_{\max }=0.2$ are both for the single-mode case: the reason they differ is that the mass is smaller for the latter case. The other trajectories show what happens as $l_{\max }$ is then increased for $L=10$. We see that both the real and imaginary parts of $T_{1}$ decrease, and the trajectory also becomes more complicated. 


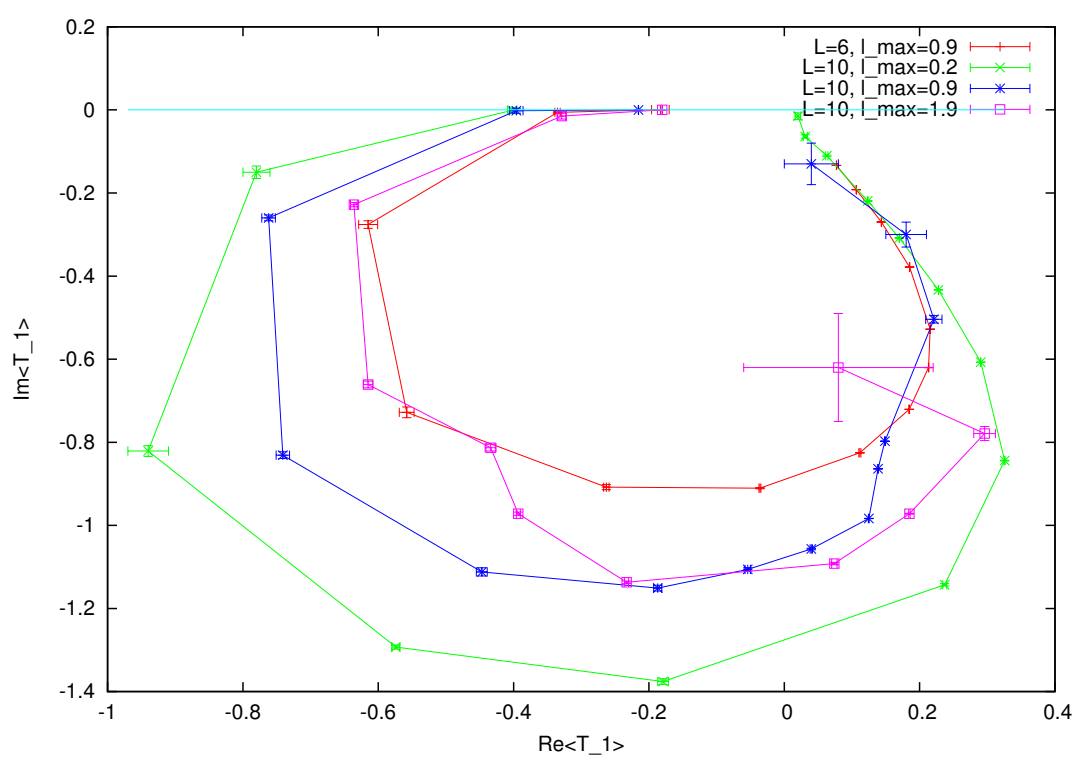

Figure 18. Trajectory of $\left\langle T_{1}\right\rangle$ in the complex plane for $L=6, l_{\max }=0.9$ (red), and for $L=10$ with $l_{\max }=0.2$ (green), $l_{\max }=0.9$ (blue), and $l_{\max }=1.9$ (magenta). Increasing $x_{\min }$ corresponds to anticlockwise movement along the trajectory.

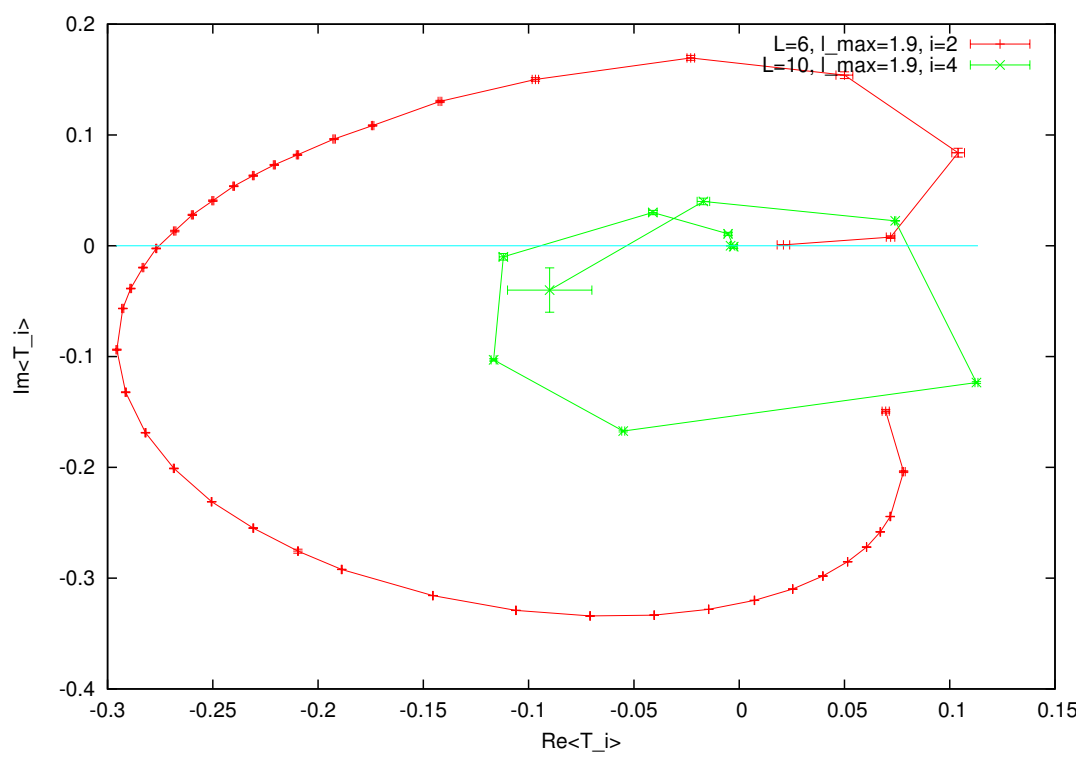

Figure 19. Trajectory of $\left\langle T_{2}\right\rangle$ in the complex plane for $L=6$ (red), and of $\left\langle T_{4}\right\rangle$ for $L=10$ (green), both for $l_{\max }=1.9$. Increasing $x_{\min }$ corresponds to anticlockwise movement along the trajectory.

Next we compare two modes with roughly the same wavelength, namely $T_{2}$ at $L=6$ and $T_{4}$ at $L=10$, both for $l_{\max }=1.9$. Their trajectories in the complex plane are plotted in figure 19. We see in this case that there is a large change in scale. Apart from this we see that whereas the $L=6$ trajectory has one loop, the trajectory for $L=10$ has at least the start of a second. 


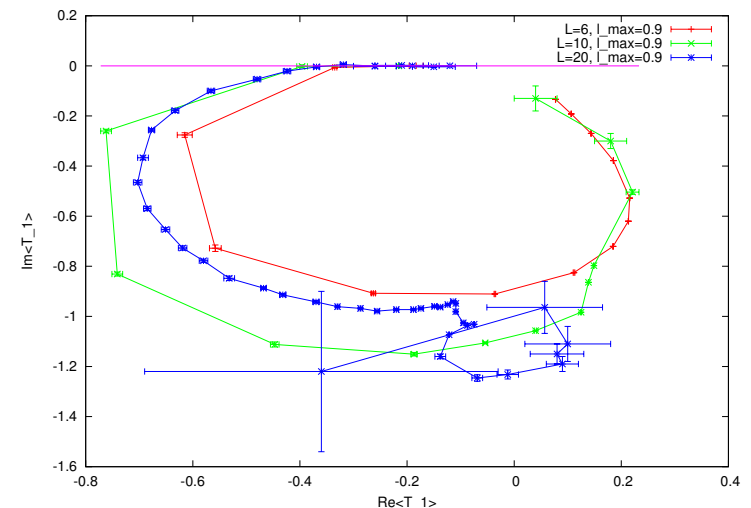

(a) $l_{\max }=0.9$.

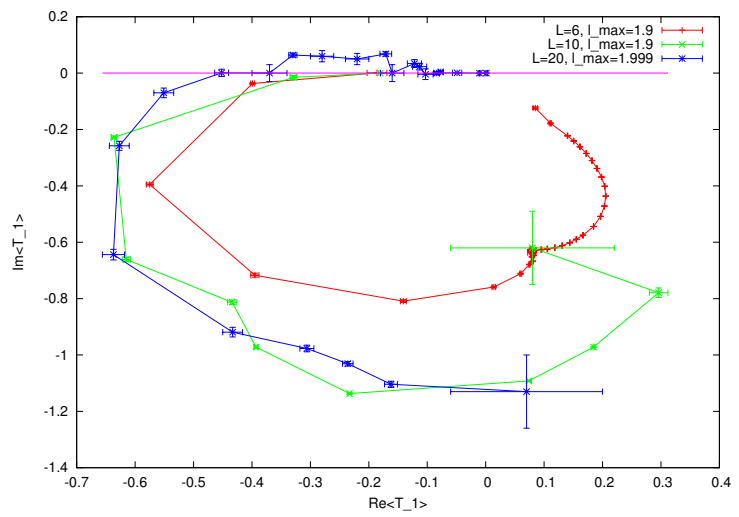

(b) $l_{\max }=1.999$.

Figure 20. Trajectory of $\left\langle T_{1}\right\rangle$ in the complex plane for $L=6$ (red), $L=10$ (green), and $L=20$ (blue). Increasing $x_{\min }$ corresponds to anticlockwise movement along the trajectory.

Keeping instead the mode number fixed, we plot $\left\langle T_{1}\right\rangle$ in figure 20 for $L=6,10$, and 20 . We see that now there is quite good matching in the magnitudes of $\left\langle T_{1}\right\rangle$. (The fact that the trajectory looks smoother for some cases is simply because we have made measurements closer together in $x_{\mathrm{min}}$.) There is no sign of the imaginary parts decreasing, or of the approach to the origin occurring at a smaller angle to the real axis. However, we once again see that trajectory becomes more complicated as $L$ increases, with e.g. two cusps and a loop being present for $L=20$ with $l_{\max }=0.9$. It also appears that the behaviour for the two values of $l_{\max }$ is similar, though unfortunately it is not possible to go to high enough $x_{\min }$ with $l_{\max }=1.999$ to see well the approach to the origin. For the same reason, we cannot really see if the trajectories are becoming more complicated.

We now turn to comparisons of the $\left\langle T_{n}^{2}\right\rangle$. As an example we plot the lowest mode $\left\langle T_{1}^{2}\right\rangle$ in figure 21. The cases $L=6, l_{\max }=0.9$ and $L=10, l_{\max }=0.2$ in this plot both have only a single mode; the large increase in $\left\langle T_{1}^{2}\right\rangle$ is due to the mass decrease. The other two trajectories then show what happens when $L$ is kept fixed and $l_{\max }$ is increased. We see that $\left\langle T_{1}^{2}\right\rangle$ decreases, which roughly means that the fluctuations of $\left\langle T_{1}\right\rangle$ decrease. Also the trajectories become more complicated, although they do not seem to be as complicated as the trajectories of the $\left\langle T_{n}\right\rangle$ we saw above.

We find similar behaviour for the other modes $\left\langle T_{n}^{2}\right\rangle$ and also for $\left\langle A_{n}^{2}\right\rangle$ : generally there are some complicated trajectories in the complex plane, with in some cases cusps or loops appearing. There is no trend for imaginary parts to get smaller or the approach to the origin to occur at a smaller angle to the real axis as we increase $L$ or $l_{\max }$.

\subsection{Relations among different expectation modes $\left\langle\boldsymbol{T}_{n}\right\rangle$}

Apart from looking at how an individual mode, say $\left\langle T_{1}\right\rangle$ moves in the complex plane as $x_{\text {min }}$ increases, we can get additional information by examining how all the modes move together. We show an example for $L=20$ for a range of $x_{\min }$ from -21 to -18 , i.e. over the transition from weak to strong coupling, with $l_{\max }=1.999$, in figure 22 . The behaviour appears to be quite complicated, with the $\left\langle T_{n}\right\rangle$ all moving off the real axis as 


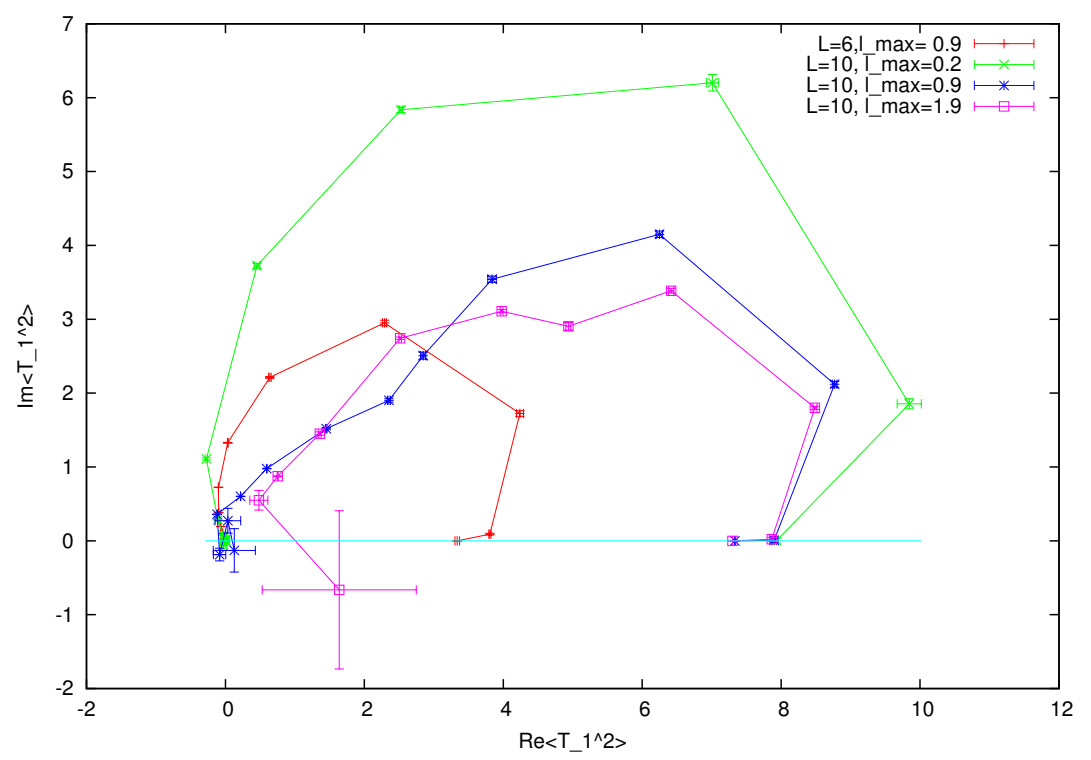

Figure 21. As figure 18, but for $\left\langle T_{1}^{2}\right\rangle$ rather than $\left\langle T_{1}\right\rangle$.

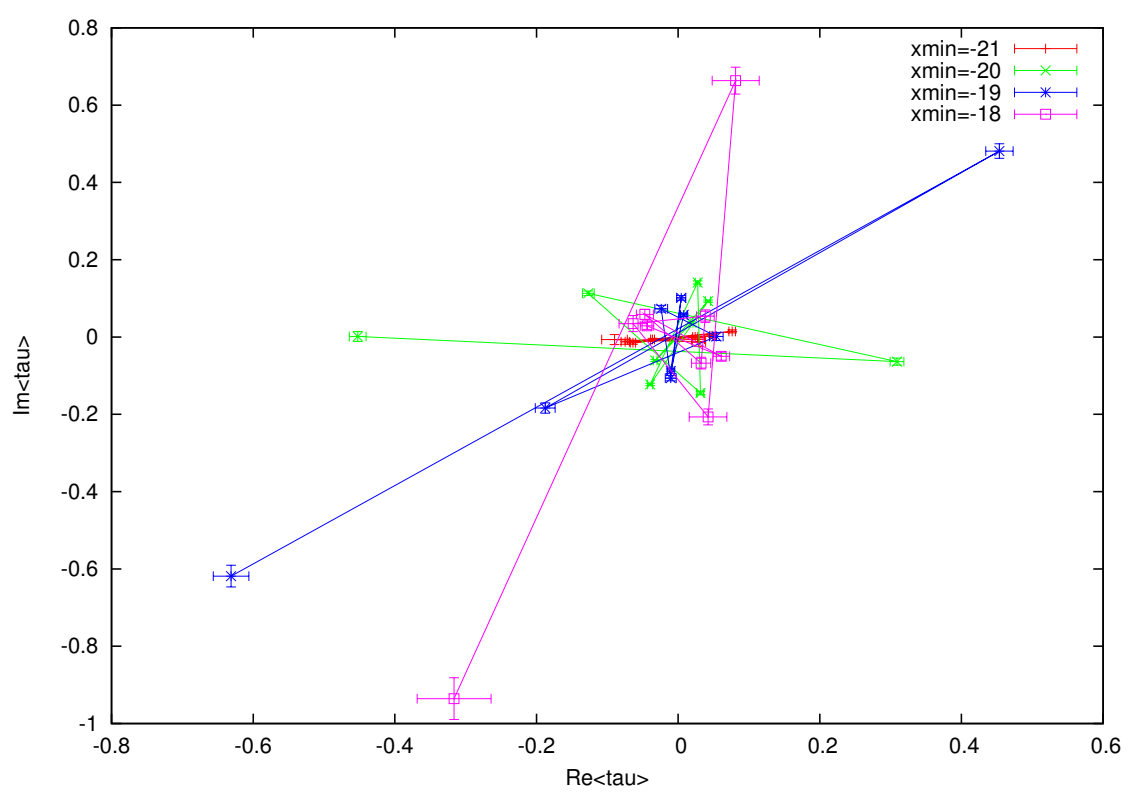

Figure 22. The $\left\langle T_{n}\right\rangle$ in the complex plane for $L=20, l_{\max }=1.999$, and various $x_{\min }$. In each case $\left\langle T_{1}\right\rangle$ is the point furthest to the left/bottom.

$x_{\text {min }}$ is increased, and then forming a complicated star-shaped pattern. We see similar behaviour for other values of the parameters.

We do not understand the full behaviour here, but some features can be understood. Firstly there is a rougly oscillatory behaviour, with $\left\langle T_{n+1}\right\rangle$ generally being on the opposite side of the origin to $\left\langle T_{n}\right\rangle$. This is presumably because the cubic terms change sign when $n$ increases by one. This in turn is because the cubic terms are proportional to integrals whose values are dominated by contributions near $x_{\max }$, and the sine-wave modes will 


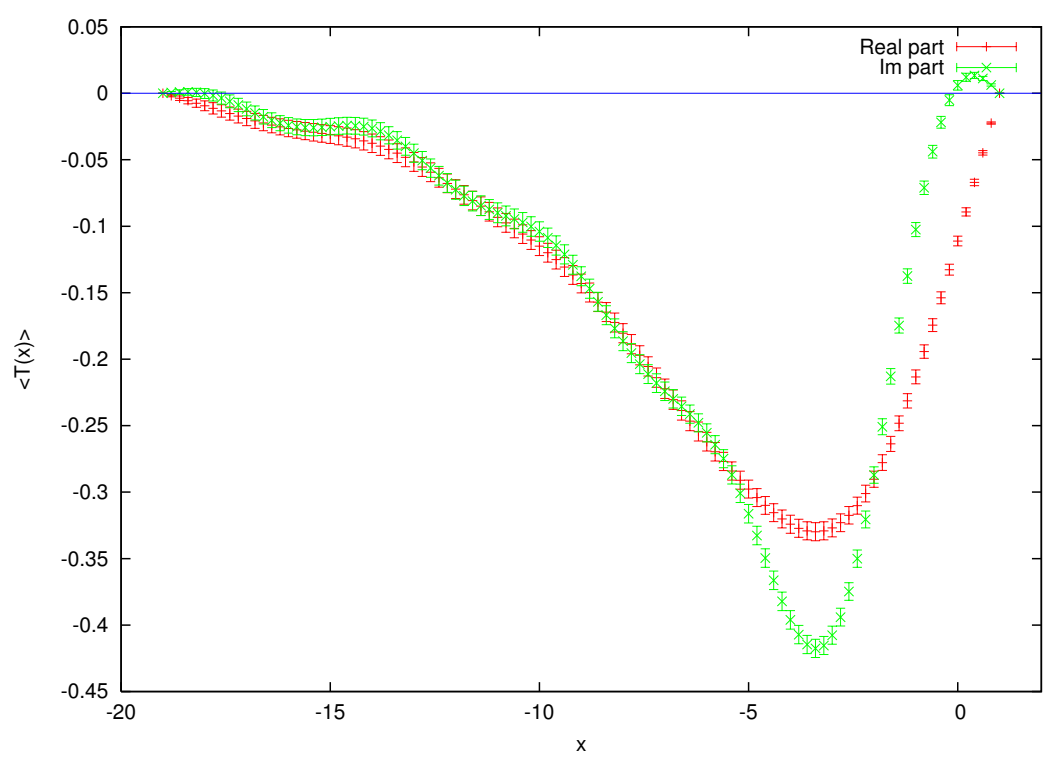

Figure 23. The real (red) and imaginary (green) parts of $\langle T(x)\rangle$ for $L=20, l_{\max }=1.999$, $x_{\min }=-19$.

change sign in this region when $n$ increases by one. Secondly there is a general decrease in magnitude as $n$ increases. This is presumably because the masses of the modes increase, constraining them to be closer to the origin.

\subsection{1 $T(x)$ in position space}

Another way to look at the behaviour of the $T_{n}$ is to recall that they are the Fourier modes of the field $T$. Thus we can translate back to position space to obtain the expectation value of $T(x)$ :

$$
\langle T(x)\rangle=\sqrt{\frac{2}{L}} \sum_{j=1}^{n_{0}}\left\langle T_{j}\right\rangle \sin \left(\frac{j \pi\left(x-x_{\min }\right)}{L}\right) .
$$

This will in general be complex of course.

The advantage of this is that the complicated behaviour of the $T_{n}$ in the complex plane can be packaged into a pair of functions, the behaviour of which may be easier to understand. We show an example in figure 23, where we plot $\langle T(x)\rangle$ for $L=20$, $l_{\max }=1.999$ and $x_{\min }=-19$, which is one of the cases plotted in figure 22.

The behaviour indeed appears simpler when plotted in this way. We see that $T(x)=0$ as both ends of the interval, as it must with our boundary conditions. Away from the boundaries it is very assymetric, with a maximum near the large- $x$ end. This is not surprising, since the cubic terms that pull $T(x)$ away from zero are largest there. Note that in this case $T$ has 9 modes, so the shortest lengthscale that can appear is roughly $L / 9 \approx 2.2$, which is comparable to the distance from the peak of $T$ to the boundary; the peak is as far to the right as it can be. Indeed, we have found that this overall shape is typical. 


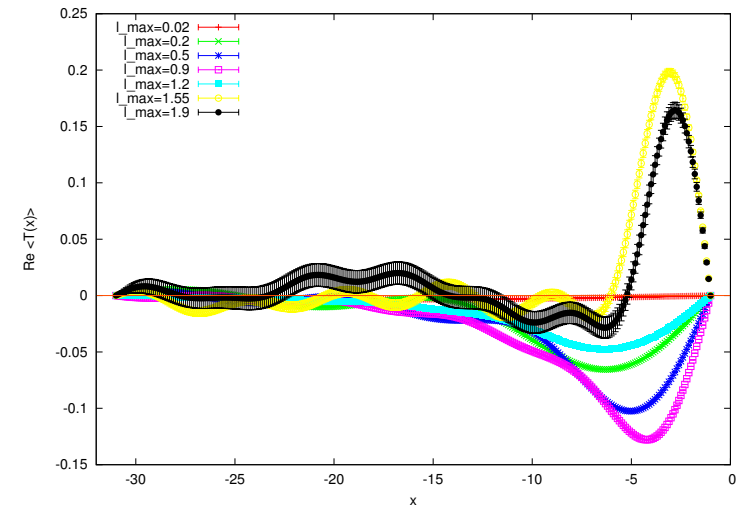

(a) Real parts.

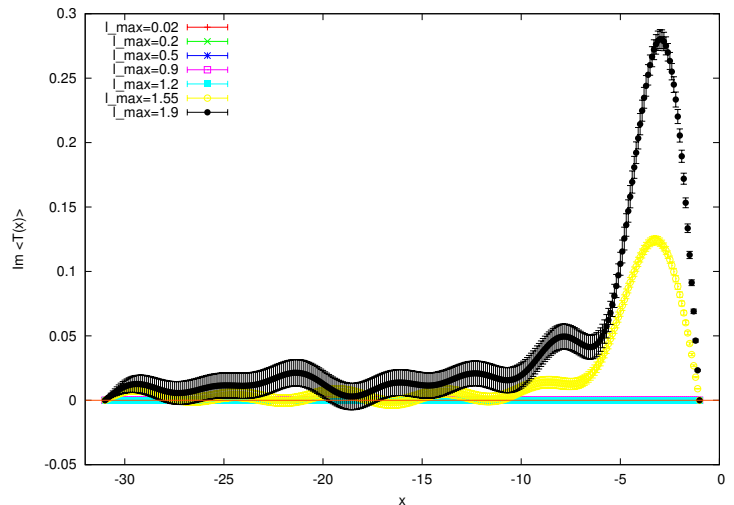

(b) Imaginary parts.

Figure 24. $\langle T(x)\rangle$ for $L=30, x_{\min }=-31$, and various values of $l_{\max }$.

This in fact raises a general issue with our simulations. The characteristic lengthscale over which we expect the fields to vary is something like $1 / V \approx 0.5$. But the finest scale we can probe is, say, half a wavelength of the highest mode. We have $n_{0}=L \sqrt{l_{\max }} / \pi$ modes on an interval of length $L$, so the highest mode has half-wavelength $\pi / \sqrt{l_{\max }}$. We can only go up to $l_{\max }=2$, giving a minimum lengthscale of $\pi / \sqrt{2} \approx 2.2$. To reach a lengthscale of 0.5 , we would need to go up to $l_{\max }=39$, probably an impossible task.

One way to look at this issue is to fix everything except $l_{\max }$ and see how things change as $l_{\max }$ increases, which corresponds to being able to look at smaller and smaller lengthscales. We have done this for the case of $L=30, x_{\min }=-31$ in figure 24 .

We see that as $l_{\max }$ increases features on smaller length-scales appear. However, there does not appear to be a smooth limit: $\langle T(x)\rangle$ changes significantly every time $l_{\max }$ is increased. In fact this should not be surprising as we know from section 4.2 that the observables depend strongly on $l_{\max }$. This suggests that we may indeed not be resolving small enough lengthscales.

\subsubsection{Correlations}

It is also possible to look at correlations among the $T_{n}$, or equivalently between $T(x)$ at different points. These are related by

$$
\left\langle T\left(x_{1}\right) T\left(x_{2}\right)\right\rangle=\frac{2}{L} \sum_{n, k=1}^{n_{0}}\left\langle T_{n} T_{k}\right\rangle \sin \left(\frac{n \pi\left(x-x_{\min }\right)}{L}\right) \sin \left(\frac{k \pi\left(x-x_{\min }\right)}{L}\right) .
$$

In principle, correlators could be used to extract the masses of the states in the theory. However, since everything is space-dependent, this will be complicated; the correlators will not simply decrease as $\sum_{k} e^{-m_{k} x}$. We have not attempted to extract masses but have simply looked at a few correlators as a first step in this direction.

We show an example in figure 25 . Here we have fixed $x_{1}$ and let $x_{2}$ vary, and subtracted $\left\langle T\left(x_{1}\right)\right\rangle\left\langle T\left(x_{2}\right)\right\rangle$ to show just the fluctuations. The results look reasonable: the largest correlations are at small separations (around $x_{2}=-3$ ). They then fall off with distance, although rather slowly. One thing to note is that the correlators are not symmetrical in space - as expected since the action is $x$-dependent. 


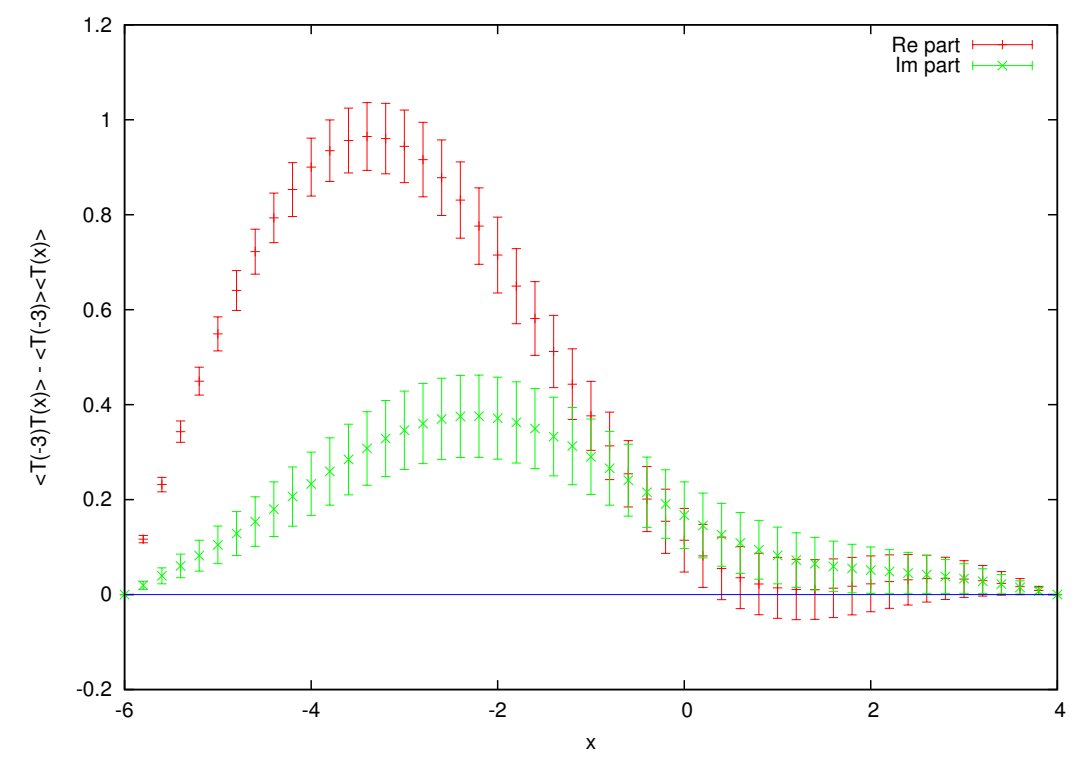

Figure 25. Correlator $\left\langle T\left(x_{1}\right) T\left(x_{2}\right)\right\rangle-\left\langle T\left(x_{1}\right)\right\rangle\left\langle T\left(x_{2}\right)\right\rangle$ for fixed $x_{1}=-3$, with $x_{\min }=-6, L=10$, and $l_{\max }=-1.9$.

\subsection{Varying the dilaton gradient}

In the full theory, the dilaton gradient $V$ is fixed to

$$
V=-\sqrt{\frac{25}{6 \alpha^{\prime}}}
$$

Let us define

$$
v \equiv 6 \alpha^{\prime} V^{2}
$$

Thus, the correct value for the dilaton slope is obtained at

$$
v=25 \text {. }
$$

However, in our level-truncated model we can choose any value, and it would be interesting to see whether anything special happens at $v=25$. In particular, we might hope that the instability will be minimised at this value.

Another important value is $v=24$. Here the mass-squared of the $T$ field, given by

$$
\alpha^{\prime} m_{0}^{2}=\frac{v}{24}-1
$$

becomes zero, and it becomes negative when $v$ is further decreased. Hence for $v<24$ we should expect a tachyonic instability, different from the cubic instability we have been concerned with before. ${ }^{15}$ This quadratic instability cannot be controlled by our analytic continuation with $\gamma=\pi / 6$, and we find the simulations indeed become unstable. We have carried out several scans in $v$ which we describe below.

\footnotetext{
${ }^{15}$ Actually for finite $L$ the lightest $T$-mode has mass-squared $\alpha^{\prime} m_{0}^{2}=\frac{v}{24}-1+\alpha^{\prime}\left(\frac{\pi}{L}\right)^{2}$ so the instability starts slightly below $v=24$.
} 


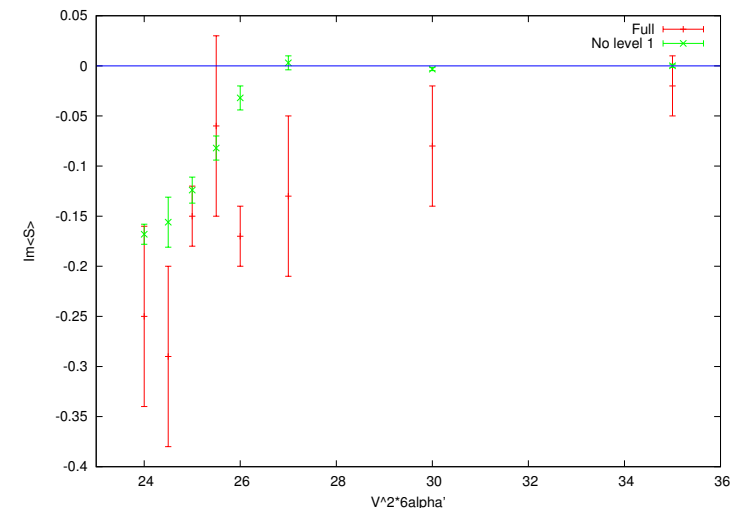

(a) $x_{\min }=-20$.

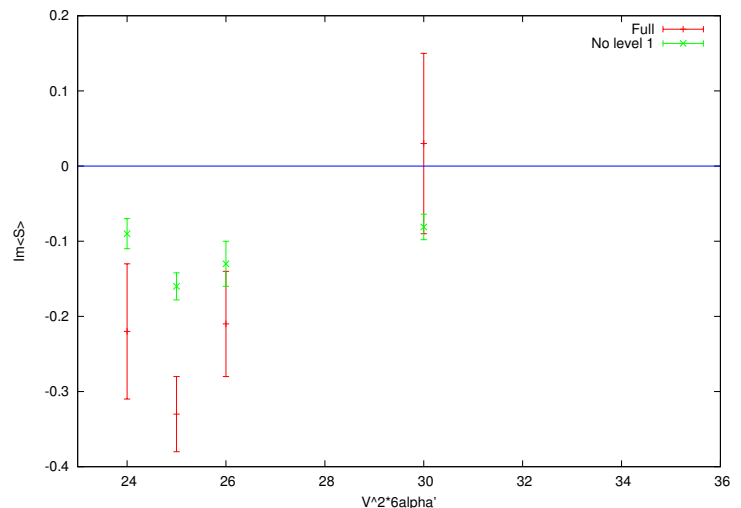

(b) $x_{\min }=-19$.

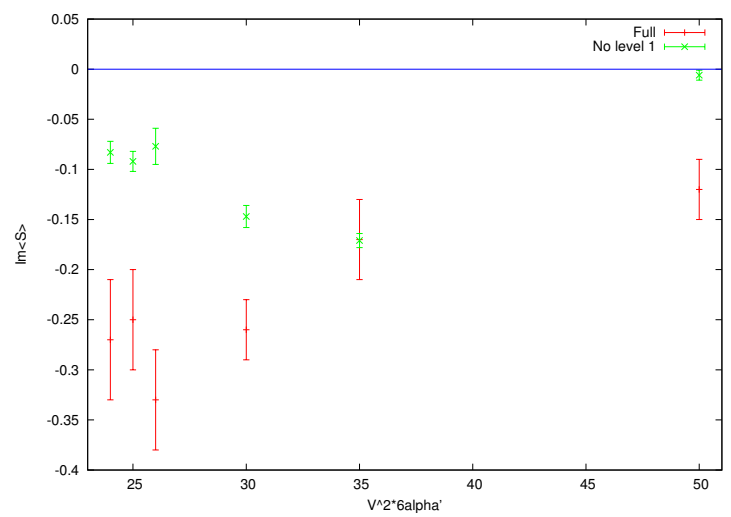

(c) $x_{\min }=-18$.

Figure 26. $\Im\langle S\rangle$ as a function of $v$ for $L=20, x_{\min }=-20,-19,-18$, and $l_{\max }=1.999$.

\subsection{1 $L=20$}

Here we have results for $l_{\max }=1.999$, and also for $l_{\max }=1.999$ without level- 1 fields. The imaginary parts of $\langle S\rangle$ and $\left\langle T_{1}\right\rangle$ are shown in figures 26 and 27 respectively. For both observables it appears that the instability decreases monotonically as $v$ increases. It is also clear that $v=25$ is no better than neighbouring values of $v$.

\subsection{2 $L=10$}

We now turn to $L=10$ where the errors are smaller. Here we use $l_{\max }=1.9$ which is again the highest value of $l_{\max }$ below 2 . We begin with $x_{\min }=-10$ which is quite weak coupling. We plot $\Im\langle S\rangle$ in figure 28 and $\Im\left\langle T_{1}\right\rangle$ in figure 29 .

Again the overall trends are similar and nothing remarkable happens at $v=25$. For the case $x_{\min }=-6$, which is extremely strong coupling, we have done a more extensive scan in $v$, going up to $v=400$. (Note the $x$-axis is compressed in these plots compared to the earlier plots in this section.) Here we see the behaviour is more complicated: there are two minima in $\Im\langle S\rangle$, and $\Im\left\langle T_{1}\right\rangle$ crosses zero and approaches zero from above for large $v$, although this does not happen if level-1 fields are excluded. Disappointingly most of this interesting behaviour is at large $v$, far from $v=25$. 


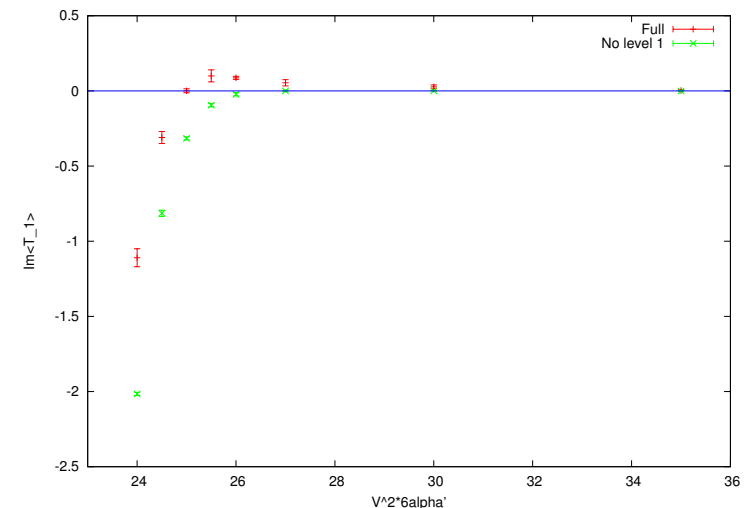

(a) $x_{\min }=-20$.

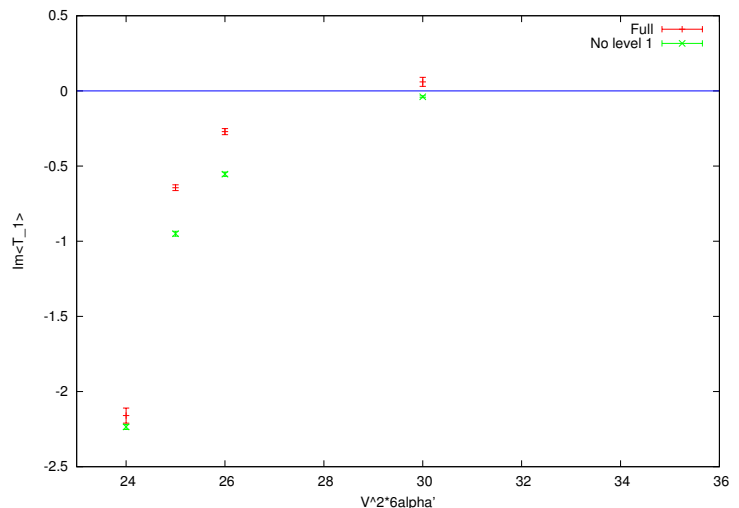

(b) $x_{\min }=-19$.

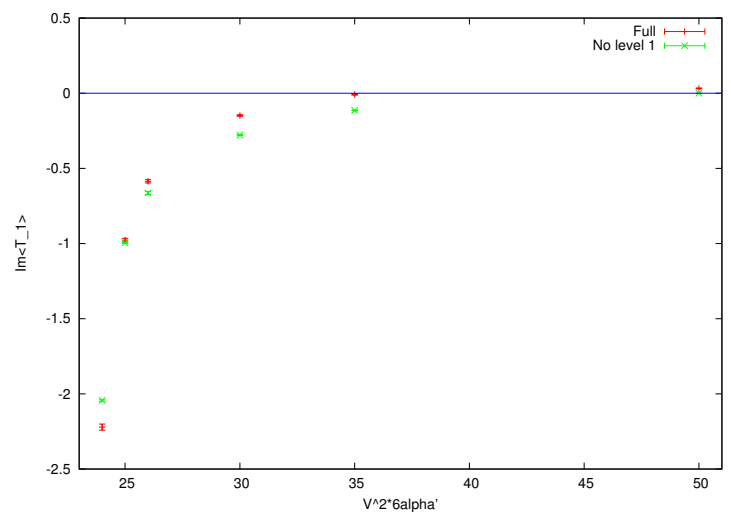

(c) $x_{\min }=-18$.

Figure 27. $\Im\left\langle T_{1}\right\rangle$ as a function of $v$ for $L=20, x_{\min }=-20,-19,-18$, and $l_{\max }=1.999$.

\subsubsection{Interpretation}

Much of the rather complicated behaviour seen above can be understood, at least qualitatively, by considering how the quadratic and cubic terms depend on $v$.

The behaviour of the quadratic (mass) terms is the more straightforward. The mass of the $T$ field (4.14) simply increases with $v$.

The cubic terms are more complicated. There are many of these, given by the coefficients $f_{i_{1} i_{2} i_{3}}$ and $g_{i_{1} i_{2} i_{3}}$. We have plotted them in figure 30 as functions of $v$ for two of the sets of parameters above: $L=10, x_{\min }=-10$ and $L=10, x_{\min }=-6$. We see that for $x_{\min }=-10$, the coefficients mostly decline throughout the range of $v$, whereas for $x_{\min }=-6$, they peak around $v=100$ and only then begin to decline.

We can understand this analytically for the particular case of $f_{111}$. Taking the exact expression and keeping only those terms which survive when $v$ is large, one obtains

$$
f_{111} \propto K^{-3 v / 24} e^{x_{\max } \sqrt{\frac{v}{24}}} .
$$

For sufficiently large $v$ the first term will dominate, so the coefficient will decline. For somewhat smaller $v$ the second, growing, term will matter as well, and there will be a peak at $v=\frac{8}{3} x_{\max }^{2} / \ln K \approx 10 x_{\max }^{2}$. However we will not see this peak if $x_{\max }$ is small - it would be at small or negative $v$ and we are only interested in $v \geq 24$ where the quadratic terms 


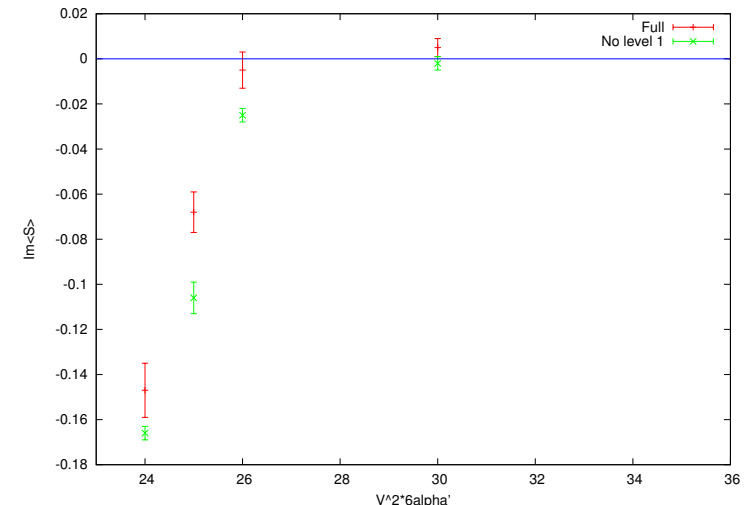

(a) $x_{\min }=-10$.

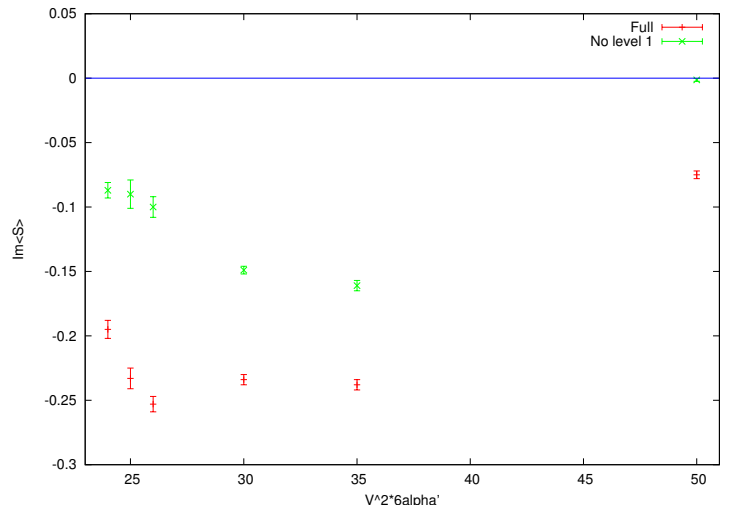

(b) $x_{\min }=-8$.

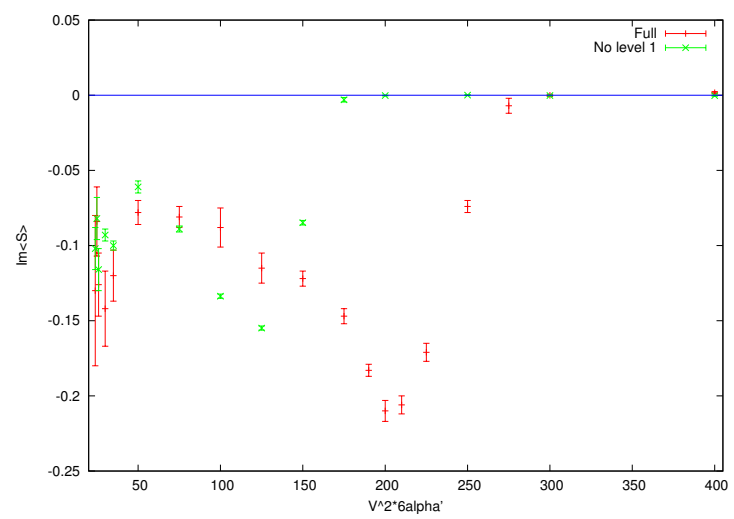

(c) $x_{\min }=-6$.

Figure 28. $\Im\langle S\rangle$ as a function of $v$ for $L=10, x_{\min }=-10,-8,-6$, and $l_{\max }=1.9$.

are stable. ${ }^{16}$ Presumably something similar happens for the other $f_{i_{1} i_{2} i_{3}}$ and the $g_{i_{1} i_{2} i_{3}}$, and this accounts for the behaviour seen in figure 30 .

Putting all this together we can understand the observed pattern of stability. For small $x_{\text {min }}$ the quadratic terms increase and the cubic terms decrease monotonically as $v$ increases. Both these trends increase stability, so we see the imaginary parts of observables decrease. However, for larger $x_{\min }$ at first both terms increase. Near $v=24$ the increase in the quadratic term should matter more, since below this value it is negative. Thus at first the instability decreases. Then the increase in the cubic terms becomes more important, and the instability increases. Finally the cubic terms peak and start decreasing, and the instability decreases again. This matches the behaviour seen for $x_{\min }=-10$. It does not fully explain the behaviour seen for $x_{\min }=-6$, but this argument is rather rough and could be refined - for example we have not considered the fermion determinant at all.

To summarise, both our numerical results and our analysis of the behaviour of the coefficients show that nothing special happens at $v=25$. It is of course possible that this will change if we worked at higher $l_{\max }$ or $L$, especially in light of the observation made in 4.6.1, according to which we are far from being able to sample space in a high enough resolution as compared to the scale set by the dilaton slope.

\footnotetext{
${ }^{16}$ Also in this case we would have to take into account terms ignored in (4.15).
} 


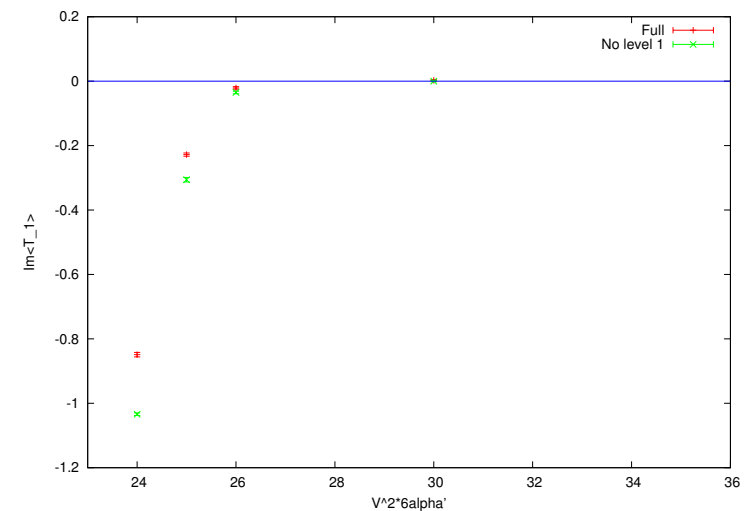

(a) $x_{\min }=-10$.

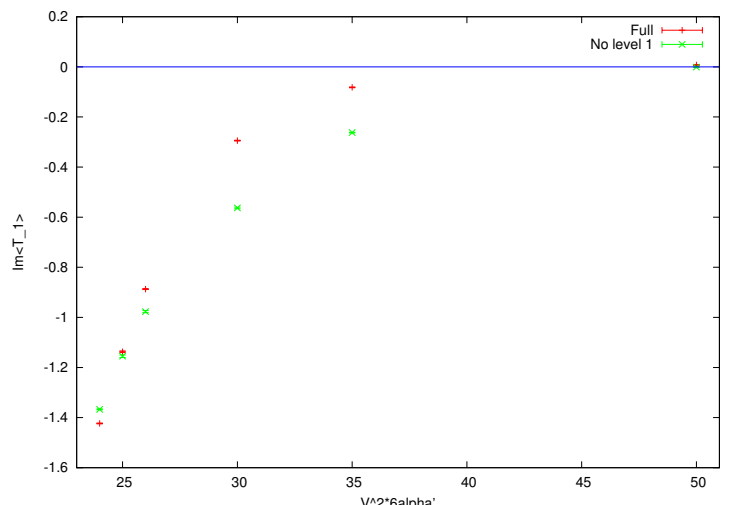

(b) $x_{\min }=-8$.

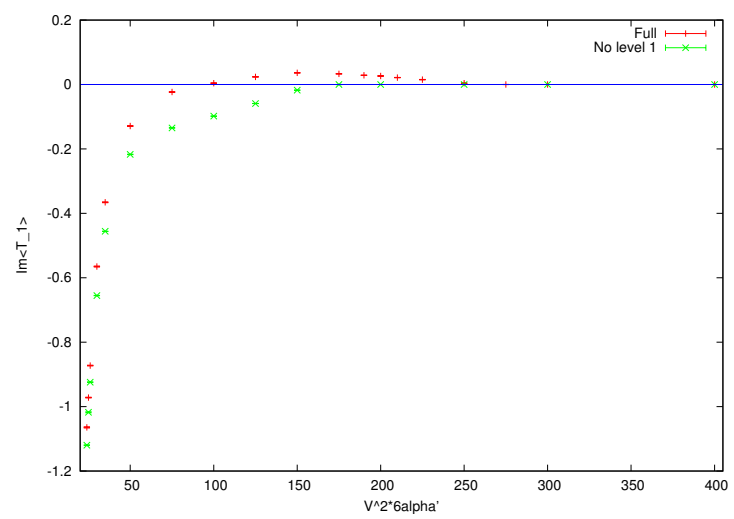

(c) $x_{\min }=-6$.

Figure 29. $\Im\left\langle T_{1}\right\rangle$ as a function of $v$ for $L=10, x_{\min }=-10,-8,-6$, and $l_{\max }=1.9$.

\subsection{Adding trivial parts to the action}

As discussed in section 2.9, we can add terms proportional to $\left(p_{1}+p_{2}+p_{3}-i V / 2\right)$, which vanish using the delta function, to the action. These should not affect the results.

Clearly there are many terms of this form we could test. We have chosen the specific example

$$
S_{\text {trivial }}=Z \sum_{n_{1,2,3}=1}^{N}\left(p_{1}+p_{2}+p_{3}-i V / 2\right) p_{1}^{4} p_{2}^{4} p_{3}^{4} T\left(p_{1}\right) T\left(p_{2}\right) T\left(p_{3}\right) .
$$

To keep this term simple, we have only considered it when only level-0 fields are present, i.e. for $l_{\max }<1$. Note that the extra cubic coefficients due to this term are similar in magnitude to the existing ones when $Z$ is of order unity.

We have found that adding this term can significantly affect the results. We show an example of its effect in figure 31. Not surprisingly, the difference between simulations with and without (4.16) increase with $Z$. However, we are more interested in how this difference depends on the other parameters, and in particular, whether it decreases in the continuum and/or large-volume limits. 


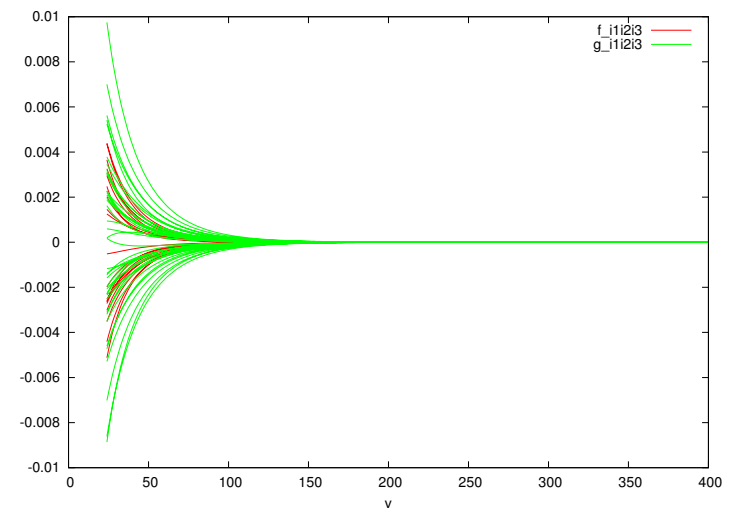

(a) $x_{\min }=-10$.

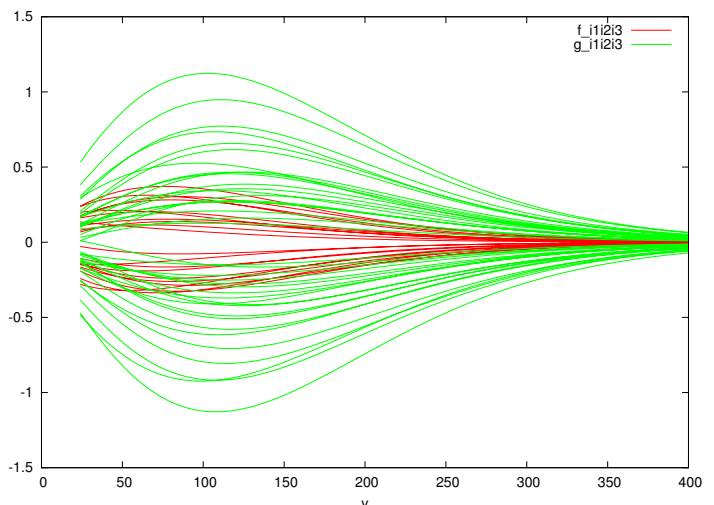

(b) $x_{\min }=-6$.

Figure 30. The $f_{i_{1} i_{2} i_{3}}$ and $g_{i_{1} i_{2} i_{3}}$ as functions of $v$ for $L=10, x_{\min }=-10,-6$.

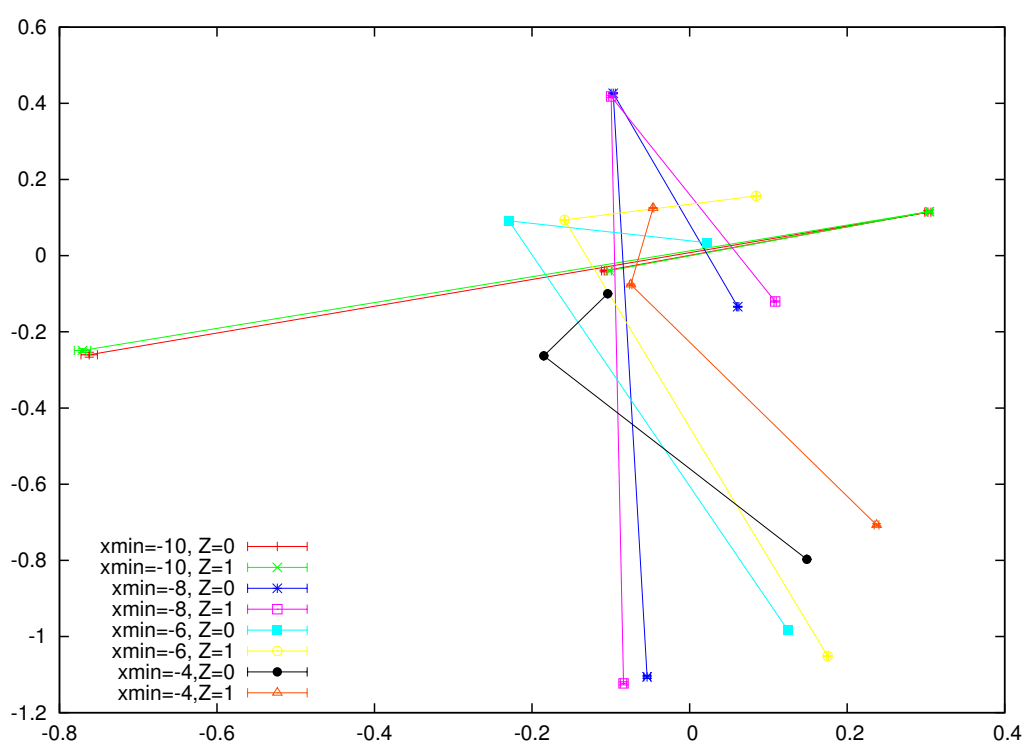

Figure 31. The $\left\langle T_{n}\right\rangle$ in the complex plane for $L=10$ and $l_{\max }=0.9$, for various $x_{\min }$, both with and without the term in (4.16).

To analyse this, we have compared results with $Z=0$ and $Z=1$ with several sets of parameters. In general, the full effect of (4.16) is complicated, but there are some clear trends:

- The differences between $Z=0$ and $Z=1$ increase at larger $x_{\min }$ (stronger coupling). This is presumably simply because the new term increases exponentially in $x$, just like the original cubic terms.

- The absolute differences are roughly similar for different mode numbers. Since the $\left\langle T_{n}\right\rangle$ are usually smaller for higher modes this means the relative differences increase.

- The differences remain roughly constant as $L$ increases. 
This last point is presumably because the dependence on $L$ is mainly in the terms $p_{i}^{4}=\frac{\left(n_{i} \pi\right)^{4}}{L^{4}}$, and the numerator and denominator cancel, since the highest mode number $n_{i}$ available is proportional to $L$ (for fixed $l_{\max }$ ).

We conclude that we cannot really establish $Z$-independence and can only continue with a rough principle of retaining the vertex in a form that is "as simple as possible". The reason for this awkward situation is the sine-expansion that we were forced to use in light of the fact that the linear dilaton prevents us from using periodic boundary conditions. Moreover, at $l_{0} \geq 2$ the sine-expansion leads to non-real coefficient terms, which after the analytical continuation lead to terms that are cubic, but not purely imaginary. This situation prevents us from carrying out our analysis in higher levels.

\subsection{Scheme 1}

We now turn to the other 'schemes', starting with scheme 1 . This is the same as scheme 4 except that the Grassmann-odd fields $B$ and $C$ are not present, but the Grassmann-even field $\mathcal{B}$ is added instead. The action in this scheme is given in (2.83) and (2.84). Note that for $0 \leq l_{\max }<1$ it is the same as scheme 4 .

We have done only a few runs to compare with the results of scheme 4. Specifically, we have carried out a scan in $x_{\text {min }}$ with the other parameters fixed to $\alpha^{\prime}=1, V=-\sqrt{\frac{25}{6 \alpha^{\prime}}}$, $L=20$, and with $l_{\max }=1.999$, since this should have the largest difference to scheme 4 . As will be seen below the instability is greater in scheme 1 at the same $x_{\min }$; in fact it is so much greater that the simulations become prohibitively expensive around $x_{\min }=-22$, where the instability is still tiny in scheme 4 . Hence the range of $x_{\min }$ covered in scheme 1 is -23 to -22.2 , which does not overlap with the range -22 to -18 covered in scheme 4 .

We plot the results for $\Im\langle S\rangle$ in figure 32 and for $\Im\left\langle T_{1}\right\rangle$ in figure 33, in both cases together with the corresponding scheme 4 results. In both cases it is clear that the instability is much greater, or equivalently appears at smaller $x_{\min }$, for scheme 1 . The shift in $x$ for equivalent imaginary parts is about 2 . This corresponds to a change in size of the cubic terms of roughly $e^{2 V} \approx 60$.

This is a very significant change, but it can be understood by the following argument. Consider first just the terms in the action quadratic in $A$ and $\mathcal{B}$, and the case where each field has only one mode:

$$
S=-\frac{1}{2}\left(m_{1}^{2}+\left(\frac{\pi}{L}\right)\right) A^{2}-\frac{1}{\alpha^{\prime}} \mathcal{B}^{2}-\frac{V}{\sqrt{2 \alpha^{\prime}}} A \mathcal{B} .
$$

Completing the square, and taking $\alpha^{\prime}=1$, one finds this has an almost massless mode in the direction $\mathcal{B}=-\frac{V}{2 \sqrt{2}} A$, with mass only $\frac{\pi}{L}$. Indeed, in the large-volume limit this mode becomes massless - presumably this is due to the fact that scheme 1 includes gauge degrees of freedom.

By itself this small but still positive mass would not lead to an instability. However, it does when the cubic terms are included. The reason is that there will be large fluctuations of this mode, giving large values of the fields $A$ and $\mathcal{B}$. These will then lead to the cubic terms of the form $A A T$ and $T \mathcal{B B}$ being large, much larger than they would be if this light mode was not present. Since some of these cubic terms are unstable, these instabilities 


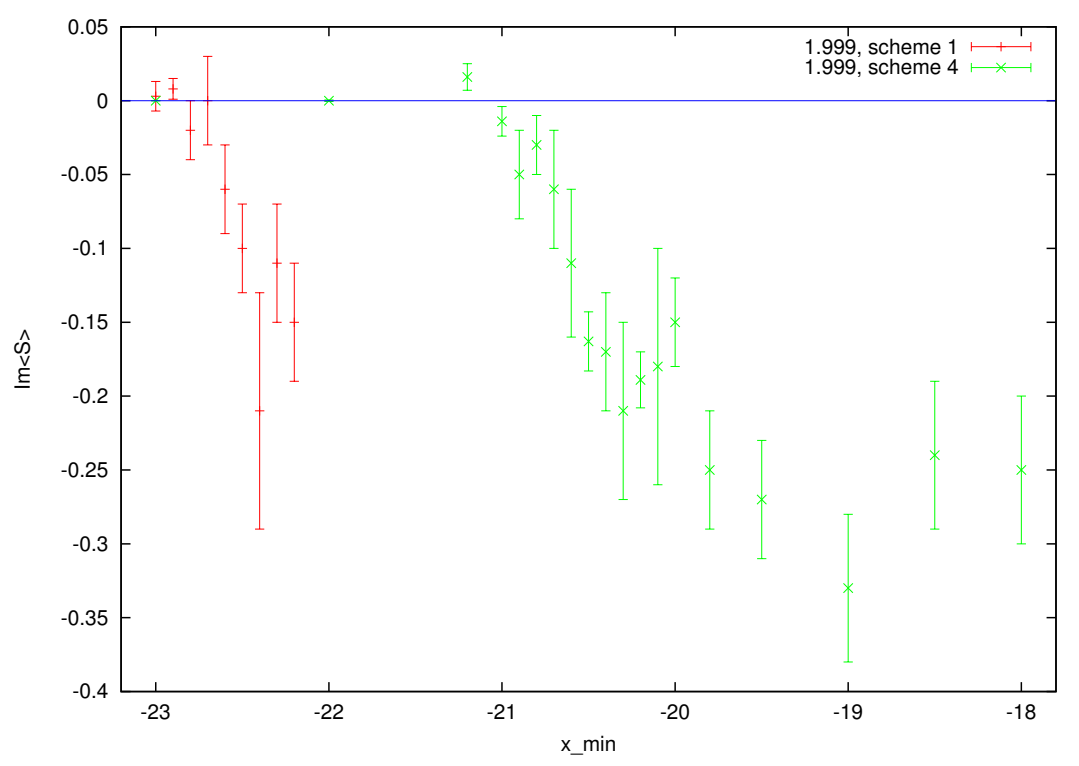

Figure 32. $\Im\langle S\rangle$ as a function of $x_{\min }$ for $L=20, l_{\max }=1.999$ for scheme 1 (red) and scheme 4 (green).

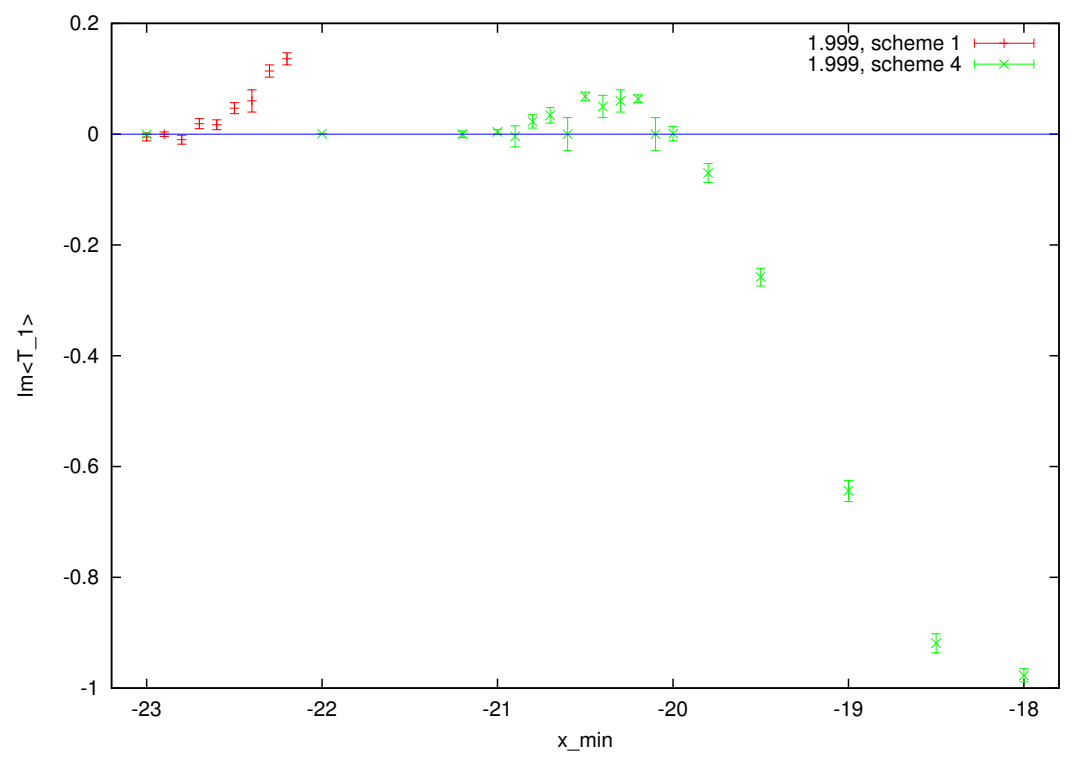

Figure 33. $\Im\left\langle T_{1}\right\rangle$ as a function of $x_{\min }$ for $L=20, l_{\max }=1.999$ for scheme 1 (red) and scheme 4 (green).

will be much larger than they would be without the light mode. As a partial check on this mechanism, we have observed that in our simulations the modes $A_{1}$ and $\mathcal{B}_{1}$ indeed fluctuate together, in exactly the direction $\mathcal{B}=-\frac{V}{2 \sqrt{2}} A$. Hence it appears that trying to included gauge degrees of freedom like this will lead to problems, and we did not continue further with simulations of scheme 1 . 


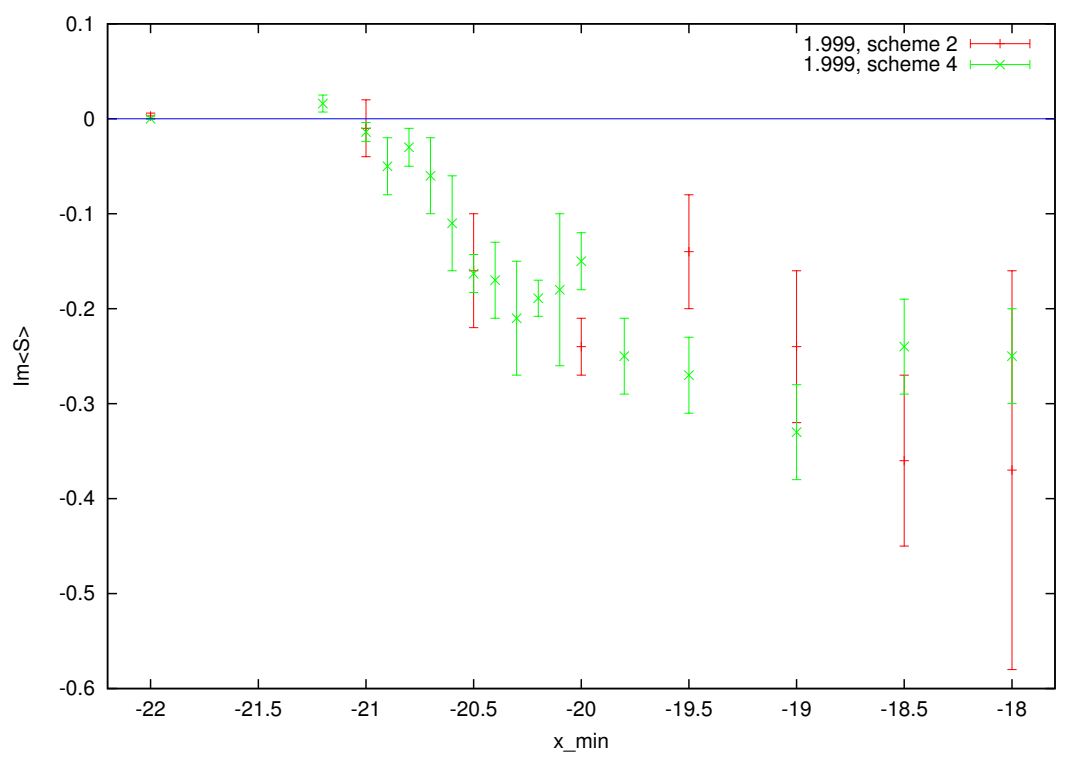

Figure 34. $\Im\langle S\rangle$ as a function of $x_{\min }$ for $L=20, l_{\max }=1.999$ for scheme 2 (red) and scheme 4 (green).

\subsection{Scheme 2}

In this section we briefly discuss our results for scheme 2 . This is the same as scheme 4 except that the Grassmann-odd fields $B$ and $C$ are not present. For $0 \leq l_{\max }<1$ it is the same as scheme 4 .

Again, we have only done simulations for a few sets of parameters, to compare the results with those for scheme 4 . Specifically, we have scanned in $x_{\min }$ for $\alpha^{\prime}=1, L=20$, $V=-\sqrt{\frac{25}{6 \alpha^{\prime}}}$, and $l_{\max }=1.999$. We have results for several $x_{\min }$ from -22 to -18 . These are plotted together with the corresponding scheme 4 results in figures 34 and 35 for $\Im\langle S\rangle$ and $\Im\left\langle T_{1}\right\rangle$ respectively.

We see that the results are very similar, with scheme 2 being perhaps slightly more unstable. This includes a region where the instability is strong so it is not just a weakcoupling phenomenon. Furthermore, the other observables are also very similar between the two schemes.

In addition to the above scan, we also found only small differences between schemes 2 and 4 for $L=10$ at several values of $V$. Thus it appears that there is not much difference between schemes 2 and 4 , at least up to $l_{\max }=2$.

\section{Discussion}

In this work we performed the first quantum non-perturbative study of a string field theory. Our aim was to estimate the feasibility of the lattice approach. In principle, a lattice string field theory could be used to examine the validity of a given string field theory, as well as to enable a numerical study of various non-perturbative aspects of string theory. One could hope to identify known as well as unknown solitons, to measure the mass of non- 


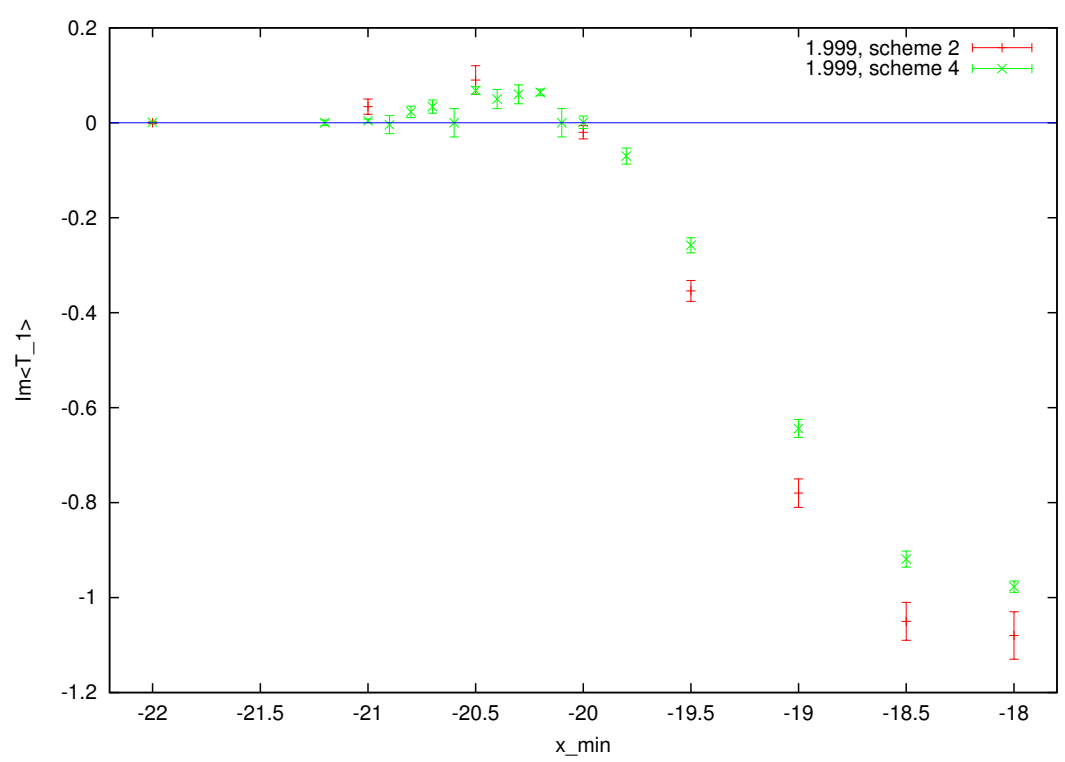

Figure 35. $\Im\left\langle T_{1}\right\rangle$ as a function of $x_{\min }$ for $L=20, l_{\max }=1.999$ for scheme 2 (red) and scheme 4 (green).

BPS objects, ${ }^{17}$ and to examine various dualities as well as other conjectures. It might also be useful for identifying and calculating generalizations of Ellwood invariants [92] (see also [93-97]).

At this stage, however, our examination was of a much more preliminary nature. We identified the technical and fundamental obstructions towards a lattice approach in string field theory, suggested possible resolutions and examined lattice simulations in order to check whether the advocated methodology could work for the simplest possible model.

While some of our results seem to suggest that our approach does make sense, others point towards further obstacles that following studies will have to face. In particular we found out that the lattice approach in the case of a linear dilaton theory must sample deep into the strong interaction regime. However, such sampling is very expensive computationally and one must look for a resolution of this problem. Possible directions include the examination of theories without linear dilatons, as well as the introduction of a Liouville wall that can potentially free us from the need to sample the strong coupling region. Another source of high computational cost is the fact that the action is complex. It could be interesting to find other ways of dealing with the original action that do not involve analytical continuation, or to find some other way to trade the analytically continued action by another, real, action. An interesting approach could be to work not with straight lines in the complex plane, but with Lefschetz Thimbles, following Witten's suggestions on the proper definition of the action of some Chern-Simons theories [98]. String field theory looks, at least superficially, very similar to Chern-Simons theory and the idea of using

\footnotetext{
${ }^{17}$ Mass shifts in string theory can be studied even without an explicit string field theoretical formulation. However, such a formulation would make the search more systematic [90, 91]. Moreover, by studying string field theory on a lattice we could also identify mass shifts due to non-perturbative effects.
} 
Lefschetz Thimbles was already implemented in lattice field theory, see e.g. [99-101]. It could also be useful to find a way to implement the Langevin method for a lattice string field theory.

A related issue is that of boundary conditions. First, the linear dilaton prevented us from using periodic boundary conditions and then it turned out that the obtained functions tend to concentrate at the rightmost part of the working segment. Again, working with different backgrounds might be useful in order to avoid this whole state of affairs. However, working with such a background might be very difficult for reasons mentioned in the introduction. The study of the theory with a Liouville wall, on the other hand, seems to be relatively simple and would probably enable us to resolve some of the difficulties we are facing. It would also be interesting to see whether the current framework is sensitive to some sort of a modification of the boundary conditions.

There could have been other reasons to object the feasibility of our approach. To begin with, we try to approximate an infinite number of fields by a truncation that takes into account only modes from a finite number of fields. Furthermore, we approximate a nonlocal action using an expansion which retains only a finite number of derivatives. While such an approach is a standard one for the description of low energy physics, it is well known that it might be inadequate for a complete description of such a theory [77, 78]. However, it is also known that a classical level truncation approach is often very accurate and useful in string field theory. Moreover, string field theory is expected to behave better than other non-local theories. On the other hand, we are dealing with a situation that is more subtle than standard level truncation computations due to the presence of the linear dilaton background, the fact that we work with more general component fields than in the usual case and, most importantly, the quantum nature of the analysis. We prefer to follow the footsteps of the original level truncation papers and examine these questions experimentally $[58,59]$.

With the introduction of higher levels new problems might be encountered. One complication is related to the fact that in string field theory some of the auxiliary fields have kinetic terms with wrong signs. This problem could be avoided by explicitly integrating out all the auxiliary fields prior to any numerical analysis. Another problem is the appearance of imaginary interaction terms. Such terms will become real after the analytical continuation and will, therefore, lead to instabilities. It seems to us that the origin of these terms is the breakdown of momentum conservation by the lattice together with the fact that this momentum conservation has an imaginary part in a linear dilaton background. It might be possible that the freedom of adding trivial terms, discussed in section 4.8, could be used for setting all the imaginary parts to zero. Exploring alternative theories and other boundary conditions, as discussed above, might be useful also in this context.

Another important issue, which we did not address in this work, is renormalization, namely comparing results at different levels. A related question is that of renormalizability. In standard field theory one of the advantages of the lattice approach is that it respects the gauge symmetry and is therefore not expected to imperil the renormalizability of the theory. In string field theory, on the other hand, gauge symmetry mixes different levels and is therefore broken by the lattice. While future works will have to study higher levels, 
they will also have to address the issues of gauge symmetry breaking, renormalizability and renormalization schemes. A possible approach that might be helpful in the context of breaking the gauge symmetry, as well as for other reasons, could be to construct a lattice in the continuous $\kappa$-basis of string field theory [102-114]. We leave this approach to future work.

Finally, one could have objected the idea of using open string field theory for such a study on the ground that it is not expected to describe the closed string moduli [115-117]. A related objection is that the theory obeys only the classical master equation, while the quantum equation is divergent [49]. This observation suggests that the theory is not really consistent at the quantum level. The difficulties with the quantum master equation most probably originate from the somewhat singular nature of Witten's star product. They are probably common also to other formulations based on this product, see e.g. [118, 119].

The singularity of the star product seems to be related to the fact that it is used in order to describe solely the open string sector. Indeed, one can consider continuous families of open-closed string field theories and Witten's theory, which is obtained as a singular limit of such an interpolation, is the only theory of the family whose master equation has quantum singularities [120]. Problems with the quantum master equation suggest that the gauge symmetry might be broken at the quantum level. However, in the case at hand it is known that at least in the Siegel gauge open string field theory leads to the correct covering of moduli spaces and to correct expressions for all amplitudes [23, 24]. Worldsheet open string theory is known to be renormalizable. Hence, as long as one uses the gauge fixed scheme we mostly used, the theory before the introduction of the lattice should be consistent quantum mechanically. Nonetheless, it would be very useful in principle to have a lattice formulation of closed string field theory [8], in which closed string moduli could also be varied. However, numerical analysis of this theory is extremely complicated already at the classical level [121-124].

To summarize, while lattice string field theory could be a useful framework, there are many obstacles on the way, some of which we dealt with in this work and others which still lie ahead. It seems that a gauge fixed approach is the most promising one. But it remains to be seen which theories can be studied and what are the most adequate backgrounds and boundary conditions. We hope that future studies will clarify these points.

\section{Acknowledgments}

We benefited from discussing the matters presented in this paper with Gert Aarts, Ted Erler, Udi Fuchs, Carlo Maccaferri, Yaron Oz, Leonardo Rastelli, Martin Schnabl and Barton Zwiebach. We would further like to thank Udi Fuchs and Carlo Maccaferri for valuable comments on a draft of the manuscript. The calculations for this work were, in part, performed on the University of Cambridge HPCs as a component of the DiRAC facility jointly funded by STFC and the Large Facilities Capital Fund of BIS.

Open Access. This article is distributed under the terms of the Creative Commons Attribution License (CC-BY 4.0), which permits any use, distribution and reproduction in any medium, provided the original author(s) and source are credited. 


\section{References}

[1] J. Polchinski, String theory: Vol. 1. An introduction to the bosonic string, Cambridge Monographs on Mathematical Physics, Cambridge University Press, Cambridge, U.K., (1998), pg. 402.

[2] K. Ohmori, A review on tachyon condensation in open string field theories, hep-th/0102085 [INSPIRE].

[3] W. Taylor and B. Zwiebach, D-branes, tachyons and string field theory, hep-th/0311017 [INSPIRE].

[4] E. Fuchs and M. Kroyter, Analytical solutions of open string field theory, Phys. Rept. 502 (2011) 89 [arXiv:0807.4722] [InSPIRE].

[5] M. Kaku and K. Kikkawa, The field theory of relativistic strings. I. Trees, Phys. Rev. D 10 (1974) 1110 [InSPIRE].

[6] M. Kaku and K. Kikkawa, The field theory of relativistic strings. II. Loops and Pomerons, Phys. Rev. D 10 (1974) 1823 [inSPIRE].

[7] E. Witten, Noncommutative geometry and string field theory, Nucl. Phys. B 268 (1986) 253 [InSPIRE].

[8] B. Zwiebach, Closed string field theory: quantum action and the B-V master equation, Nucl. Phys. B 390 (1993) 33 [hep-th/9206084] [INSPIRE].

[9] E. Witten, Interacting field theory of open superstrings, Nucl. Phys. B 276 (1986) 291 [INSPIRE].

[10] C.R. Preitschopf, C.B. Thorn and S.A. Yost, Superstring field theory, Nucl. Phys. B 337 (1990) 363 [InSPIRE].

[11] I.Y. Arefeva, P.B. Medvedev and A.P. Zubarev, New representation for string field solves the consistency problem for open superstring field theory, Nucl. Phys. B 341 (1990) 464 [INSPIRE].

[12] N. Berkovits, SuperPoincaré invariant superstring field theory, Nucl. Phys. B 450 (1995) 90 [Erratum ibid. B 459 (1996) 439-451] [hep-th/9503099] [INSPIRE].

[13] N. Berkovits, Pure spinor formalism as an $N=2$ topological string, JHEP 10 (2005) 089 [hep-th/0509120] [INSPIRE].

[14] M. Kroyter, Superstring field theory in the democratic picture, Adv. Theor. Math. Phys. 15 (2011) 741 [arXiv:0911.2962] [InSPIRE].

[15] T. Erler, S. Konopka and I. Sachs, Resolving Witten's superstring field theory, JHEP 04 (2014) 150 [arXiv:1312.2948] [INSPIRE].

[16] R. Saroja and A. Sen, Picture changing operators in closed fermionic string field theory, Phys. Lett. B 286 (1992) 256 [hep-th/9202087] [INSPIRE].

[17] Y. Okawa and B. Zwiebach, Heterotic string field theory, JHEP 07 (2004) 042 [hep-th/0406212] [INSPIRE].

[18] N. Berkovits, Y. Okawa and B. Zwiebach, WZW-like action for heterotic string field theory, JHEP 11 (2004) 038 [hep-th/0409018] [INSPIRE].

[19] H. Kunitomo, The Ramond sector of heterotic string field theory, PTEP 2014 (2014) 043B01 [arXiv: 1312.7197] [INSPIRE].

[20] B. Jurčo and K. Münster, Type II superstring field theory: geometric approach and operadic description, JHEP 04 (2013) 126 [arXiv:1303.2323] [INSPIRE]. 
[21] H. Matsunaga, Construction of a gauge-invariant action for type II superstring field theory, arXiv: 1305.3893 [INSPIRE].

[22] T. Erler, S. Konopka and I. Sachs, NS-NS sector of closed superstring field theory, JHEP 08 (2014) 158 [arXiv: 1403.0940] [INSPIRE].

[23] S.B. Giddings, E.J. Martinec and E. Witten, Modular invariance in string field theory, Phys. Lett. B 176 (1986) 362 [INSPIRE].

[24] B. Zwiebach, A proof that Witten's open string theory gives a single cover of moduli space, Commun. Math. Phys. 142 (1991) 193 [INSPIRE].

[25] A. Sen, Descent relations among bosonic D-branes, Int. J. Mod. Phys. A 14 (1999) 4061 [hep-th/9902105] [INSPIRE].

[26] A. Sen, Universality of the tachyon potential, JHEP 12 (1999) 027 [hep-th/9911116] [INSPIRE].

[27] A. Sen and B. Zwiebach, Tachyon condensation in string field theory, JHEP 03 (2000) 002 [hep-th/9912249] [INSPIRE].

[28] N. Moeller and W. Taylor, Level truncation and the tachyon in open bosonic string field theory, Nucl. Phys. B 583 (2000) 105 [hep-th/0002237] [INSPIRE].

[29] N. Berkovits, A. Sen and B. Zwiebach, Tachyon condensation in superstring field theory, Nucl. Phys. B 587 (2000) 147 [hep-th/0002211] [InSPIRE].

[30] M. Schnabl, Analytic solution for tachyon condensation in open string field theory, Adv. Theor. Math. Phys. 10 (2006) 433 [hep-th/0511286] [INSPIRE].

[31] Y. Okawa, Comments on Schnabl's analytic solution for tachyon condensation in Witten's open string field theory, JHEP 04 (2006) 055 [hep-th/0603159] [INSPIRE].

[32] E. Fuchs and M. Kroyter, On the validity of the solution of string field theory, JHEP 05 (2006) 006 [hep-th/0603195] [INSPIRE].

[33] I. Ellwood and M. Schnabl, Proof of vanishing cohomology at the tachyon vacuum, JHEP 02 (2007) 096 [hep-th/0606142] [INSPIRE].

[34] T. Erler and M. Schnabl, A simple analytic solution for tachyon condensation, JHEP 10 (2009) 066 [arXiv:0906.0979] [INSPIRE].

[35] T. Erler, Tachyon vacuum in cubic superstring field theory, JHEP 01 (2008) 013 [arXiv: 0707.4591] [INSPIRE].

[36] E. Fuchs and M. Kroyter, On the classical equivalence of superstring field theories, JHEP 10 (2008) 054 [arXiv:0805.4386] [INSPIRE].

[37] I.Y. Aref'eva, R.V. Gorbachev and P.B. Medvedev, Tachyon solution in cubic Neveu-Schwarz string field theory, Theor. Math. Phys. 158 (2009) 320 [arXiv:0804.2017] [INSPIRE].

[38] T. Erler, Analytic solution for tachyon condensation in Berkovits' open superstring field theory, JHEP 11 (2013) 007 [arXiv: 1308.4400] [INSPIRE].

[39] M. Schnabl, Comments on marginal deformations in open string field theory, Phys. Lett. B 654 (2007) 194 [hep-th/0701248] [INSPIRE].

[40] M. Kiermaier, Y. Okawa, L. Rastelli and B. Zwiebach, Analytic solutions for marginal deformations in open string field theory, JHEP 01 (2008) 028 [hep-th/0701249] [INSPIRE].

[41] E. Fuchs, M. Kroyter and R. Potting, Marginal deformations in string field theory, JHEP 09 (2007) 101 [arXiv:0704.2222] [INSPIRE]. 
[42] M. Kiermaier and Y. Okawa, Exact marginality in open string field theory: a general framework, JHEP 11 (2009) 041 [arXiv:0707.4472] [INSPIRE].

[43] T. Erler, Marginal solutions for the superstring, JHEP 07 (2007) 050 [arXiv:0704.0930] [INSPIRE].

[44] Y. Okawa, Analytic solutions for marginal deformations in open superstring field theory, JHEP 09 (2007) 084 [arXiv:0704.0936] [INSPIRE].

[45] E. Fuchs and M. Kroyter, Marginal deformation for the photon in superstring field theory, JHEP 11 (2007) 005 [arXiv:0706.0717] [INSPIRE].

[46] T. Noumi and Y. Okawa, Solutions from boundary condition changing operators in open superstring field theory, JHEP 12 (2011) 034 [arXiv:1108.5317] [INSPIRE].

[47] C. Maccaferri, A simple solution for marginal deformations in open string field theory, JHEP 05 (2014) 004 [arXiv: 1402.3546] [INSPIRE].

[48] J. Polchinski, What is string theory?, hep-th/9411028 [INSPIRE].

[49] C.B. Thorn, String field theory, Phys. Rept. 175 (1989) 1 [INSPIRE].

[50] K.G. Wilson, Confinement of quarks, Phys. Rev. D 10 (1974) 2445 [inSPIRE].

[51] M. Creutz, Monte Carlo study of quantized SU(2) gauge theory, Phys. Rev. D 21 (1980) 2308 [INSPIRE].

[52] M. Creutz, Quarks, gluons and lattices, Cambridge Monographs on Mathematical Physics, Cambridge University Press, Cambridge, U.K., (1984).

[53] H.J. Rothe, Lattice gauge theories: an introduction, World Sci. Lect. Notes Phys. 82 (2012) 1.

[54] I. Montvay and G. Münster, Quantum fields on a lattice, Cambridge Monographs on Mathematical Physics, Cambridge University Press, Cambridge, U.K., (1994).

[55] J. Smit, Introduction to quantum fields on a lattice: a robust mate, Cambridge Lect. Notes Phys. 15 (2002) 1 [INSPIRE].

[56] T. DeGrand and C.E. DeTar, Lattice methods for quantum chromodynamics, World Scientific, (2006).

[57] C. Gattringer and C.B. Lang, Quantum chromodynamics on the lattice, Lect. Notes Phys. 788 (2010) 1.

[58] V.A. Kostelecky and S. Samuel, The static tachyon potential in the open bosonic string theory, Phys. Lett. B 207 (1988) 169 [InSPIRE].

[59] V.A. Kostelecky and S. Samuel, On a nonperturbative vacuum for the open bosonic string, Nucl. Phys. B 336 (1990) 263 [INSPIRE].

[60] M. Bochicchio, Gauge fixing for the field theory of the bosonic string, Phys. Lett. B 193 (1987) 31 [INSPIRE].

[61] M. Bochicchio, String field theory in the Siegel gauge, Phys. Lett. B 188 (1987) 330 [INSPIRE].

[62] C.B. Thorn, Perturbation theory for quantized string fields, Nucl. Phys. B 287 (1987) 61 [INSPIRE].

[63] M. Bershadsky and D. Kutasov, Open string theory in $(1+1)$-dimensions, Phys. Lett. B 274 (1992) 331 [hep-th/9110034] [INSPIRE].

[64] M. Bershadsky and D. Kutasov, Scattering of open and closed strings in $(1+1)$-dimensions, Nucl. Phys. B 382 (1992) 213 [hep-th/9204049] [INSPIRE]. 
[65] I.Y. Arefeva, P.B. Medvedev and A.P. Zubarev, Interaction of $D=2, c=1$ discrete states from string field theory, Mod. Phys. Lett. A 8 (1993) 2167 [hep-th/9212156] [INSPIRE].

[66] B. Urosevic, Perturbation theory in two-dimensional open string field theory, Phys. Rev. D 47 (1993) 5460 [hep-th/9302082] [INSPIRE].

[67] B. Urosevic, Off-shell amplitudes in two-dimensional open string field theory, Phys. Rev. D 50 (1994) 4075 [hep-th/9402105] [INSPIRE].

[68] E. Witten, Ground ring of two-dimensional string theory, Nucl. Phys. B 373 (1992) 187 [hep-th/9108004] [INSPIRE].

[69] A.B. Zamolodchikov and A.B. Zamolodchikov, Liouville field theory on a pseudosphere, hep-th/0101152 [INSPIRE].

[70] V. Fateev, A.B. Zamolodchikov and A.B. Zamolodchikov, Boundary Liouville field theory. I. Boundary state and boundary two point function, hep-th/0001012 [INSPIRE].

[71] J. Teschner, Liouville theory revisited, Class. Quant. Grav. 18 (2001) R153 [hep-th/0104158] [INSPIRE].

[72] J. McGreevy and H.L. Verlinde, Strings from tachyons: the $c=1$ matrix reloaded, JHEP 12 (2003) 054 [hep-th/0304224] [INSPIRE].

[73] I.R. Klebanov, J.M. Maldacena and N. Seiberg, D-brane decay in two-dimensional string theory, JHEP 07 (2003) 045 [hep-th/0305159] [INSPIRE].

[74] S. Hellerman and M. Schnabl, Light-like tachyon condensation in open string field theory, JHEP 04 (2013) 005 [arXiv:0803.1184] [INSPIRE].

[75] F. Beaujean and N. Moeller, Delays in open string field theory, arXiv:0912.1232 [INSPIRE].

[76] A. LeClair, M.E. Peskin and C.R. Preitschopf, String field theory on the conformal plane. I. Kinematical principles, Nucl. Phys. B 317 (1989) 411 [INSPIRE].

[77] D.A. Eliezer and R.P. Woodard, The problem of nonlocality in string theory, Nucl. Phys. B 325 (1989) 389 [INSPIRE].

[78] N. Moeller and B. Zwiebach, Dynamics with infinitely many time derivatives and rolling tachyons, JHEP 10 (2002) 034 [hep-th/0207107] [INSPIRE].

[79] D.J. Gross and A. Jevicki, Operator formulation of interacting string field theory. I., Nucl. Phys. B 283 (1987) 1 [INSPIRE].

[80] D.J. Gross and A. Jevicki, Operator formulation of interacting string field theory. II., Nucl. Phys. B 287 (1987) 225 [inSPIRE].

[81] D.J. Gross and A. Jevicki, Operator formulation of interacting string field theory. III. NSR superstring, Nucl. Phys. B 293 (1987) 29 [INSPIRE].

[82] S. Samuel, The physical and ghost vertex in Witten's string field theory, Phys. Lett. B 181 (1986) 255 [INSPIRE].

[83] N. Ohta, Covariant interacting string field theory in the Fock space representation, Phys. Rev. D 34 (1986) 3785 [Erratum ibid. D 35 (1987) 2627] [InSPIRE].

[84] E. Cremmer, A. Schwimmer and C.B. Thorn, The vertex function in Witten's formulation of string field theory, Phys. Lett. B 179 (1986) 57 [InSPIRE].

[85] L. Rastelli, A. Sen and B. Zwiebach, Classical solutions in string field theory around the tachyon vacuum, Adv. Theor. Math. Phys. 5 (2002) 393 [hep-th/0102112] [INSPIRE].

[86] L. Bonora, C. Maccaferri, D. Mamone and M. Salizzoni, Topics in string field theory, hep-th/0304270 [INSPIRE]. 
[87] L. Rastelli and B. Zwiebach, Tachyon potentials, star products and universality, JHEP 09 (2001) 038 [hep-th/0006240] [INSPIRE].

[88] G. Aarts, L. Bongiovanni, E. Seiler, D. Sexty and I.-O. Stamatescu, Controlling complex Langevin dynamics at finite density, Eur. Phys. J. A 49 (2013) 89 [arXiv:1303.6425] [INSPIRE].

[89] G. Aarts, F.A. James, E. Seiler and I.-O. Stamatescu, Complex Langevin: etiology and diagnostics of its main problem, Eur. Phys. J. C 71 (2011) 1756 [arXiv:1101.3270] [INSPIRE].

[90] R. Pius, A. Rudra and A. Sen, Mass renormalization in string theory: special states, JHEP 07 (2014) 058 [arXiv:1311.1257] [InSPIRE].

[91] R. Pius, A. Rudra and A. Sen, Mass renormalization in string theory: general states, JHEP 07 (2014) 062 [arXiv: 1401.7014] [INSPIRE].

[92] I. Ellwood, The closed string tadpole in open string field theory, JHEP 08 (2008) 063 [arXiv:0804.1131] [INSPIRE].

[93] A. Hashimoto and N. Itzhaki, Observables of string field theory, JHEP 01 (2002) 028 [hep-th/0111092] [INSPIRE].

[94] D. Gaiotto, L. Rastelli, A. Sen and B. Zwiebach, Ghost structure and closed strings in vacuum string field theory, Adv. Theor. Math. Phys. 6 (2003) 403 [hep-th/0111129] [INSPIRE].

[95] T. Kawano, I. Kishimoto and T. Takahashi, Gauge invariant overlaps for classical solutions in open string field theory, Nucl. Phys. B 803 (2008) 135 [arXiv:0804.1541] [INSPIRE].

[96] M. Kiermaier, Y. Okawa and B. Zwiebach, The boundary state from open string fields, arXiv:0810.1737 [INSPIRE].

[97] M. Kudrna, C. Maccaferri and M. Schnabl, Boundary state from Ellwood invariants, JHEP 07 (2013) 033 [arXiv:1207.4785] [INSPIRE].

[98] E. Witten, Analytic continuation of Chern-Simons theory, arXiv:1001.2933 [INSPIRE].

[99] AuroraScience collaboration, M. Cristoforetti, F. Di Renzo and L. Scorzato, New approach to the sign problem in quantum field theories: high density $Q C D$ on a Lefschetz thimble, Phys. Rev. D 86 (2012) 074506 [arXiv:1205.3996] [INSPIRE].

[100] G. Aarts, Lefschetz thimbles and stochastic quantization: complex actions in the complex plane, Phys. Rev. D 88 (2013) 094501 [arXiv: 1308.4811] [INSPIRE].

[101] H. Fujii et al., Hybrid Monte Carlo on Lefschetz thimbles - A study of the residual sign problem, JHEP 10 (2013) 147 [arXiv:1309.4371] [INSPIRE].

[102] L. Rastelli, A. Sen and B. Zwiebach, Star algebra spectroscopy, JHEP 03 (2002) 029 [hep-th/0111281] [INSPIRE].

[103] K. Okuyama, Ghost kinetic operator of vacuum string field theory, JHEP 01 (2002) 027 [hep-th/0201015] [INSPIRE].

[104] M.R. Douglas, H. Liu, G.W. Moore and B. Zwiebach, Open string star as a continuous Moyal product, JHEP 04 (2002) 022 [hep-th/0202087] [INSPIRE].

[105] T. Erler, Moyal formulation of Witten's star product in the fermionic ghost sector, hep-th/0205107 [INSPIRE].

[106] E. Fuchs, M. Kroyter and A. Marcus, Squeezed state projectors in string field theory, JHEP 09 (2002) 022 [hep-th/0207001] [INSPIRE]. 
[107] E. Fuchs, M. Kroyter and A. Marcus, Virasoro operators in the continuous basis of string field theory, JHEP 11 (2002) 046 [hep-th/0210155] [INSPIRE].

[108] E. Fuchs, M. Kroyter and A. Marcus, Continuous half string representation of string field theory, JHEP 11 (2003) 039 [hep-th/0307148] [INSPIRE].

[109] T. Erler, A fresh look at midpoint singularities in the algebra of string fields, JHEP 03 (2005) 042 [hep-th/0304044] [INSPIRE].

[110] E. Fuchs and M. Kroyter, Schnabl's $\mathcal{L}_{0}$ operator in the continuous basis, JHEP 10 (2006) 067 [hep-th/0605254] [INSPIRE].

[111] E. Fuchs and M. Kroyter, Universal regularization for string field theory, JHEP 02 (2007) 038 [hep-th/0610298] [INSPIRE].

[112] L. Bonora, C. Maccaferri, R.J. Scherer Santos and D.D. Tolla, Ghost story. I. Wedge states in the oscillator formalism, JHEP 09 (2007) 061 [arXiv:0706.1025] [INSPIRE].

[113] L. Bonora, C. Maccaferri, R.J. Scherer Santos and D.D. Tolla, Ghost story. II. The midpoint ghost vertex, JHEP 11 (2009) 075 [arXiv:0908.0055] [INSPIRE].

[114] L. Bonora, C. Maccaferri and D.D. Tolla, Ghost story. III. Back to ghost number zero, JHEP 11 (2009) 086 [arXiv:0908.0056] [INSPIRE].

[115] N. Moeller and I. Sachs, Closed string cohomology in open string field theory, JHEP 07 (2011) 022 [arXiv: 1010.4125] [INSPIRE].

[116] K. Münster and I. Sachs, Quantum open-closed homotopy algebra and string field theory, Commun. Math. Phys. 321 (2013) 769 [arXiv:1109.4101] [INSPIRE].

[117] K. Münster and I. Sachs, On homotopy algebras and quantum string field theory, arXiv:1303.3444 [INSPIRE].

[118] M. Kroyter, Democratic superstring field theory: gauge fixing, JHEP 03 (2011) 081 [arXiv:1010.1662] [InSPIRE].

[119] M. Kroyter, Y. Okawa, M. Schnabl, S. Torii and B. Zwiebach, Open superstring field theory I: gauge fixing, ghost structure and propagator, JHEP 03 (2012) 030 [arXiv:1201.1761] [INSPIRE].

[120] B. Zwiebach, Interpolating string field theories, Mod. Phys. Lett. A 7 (1992) 1079 [hep-th/9202015] [INSPIRE].

[121] H. Yang and B. Zwiebach, A closed string tachyon vacuum?, JHEP 09 (2005) 054 [hep-th/0506077] [INSPIRE].

[122] N. Moeller and H. Yang, The nonperturbative closed string tachyon vacuum to high level, JHEP 04 (2007) 009 [hep-th/0609208] [INSPIRE].

[123] N. Moeller, Closed bosonic string field theory at quintic order: five-tachyon contact term and dilaton theorem, JHEP 03 (2007) 043 [hep-th/0609209] [INSPIRE].

[124] N. Moeller, Closed bosonic string field theory at quintic order. II. Marginal deformations and effective potential, JHEP 09 (2007) 118 [arXiv:0705.2102] [INSPIRE]. 